Generating Language-Based Environments

Thomas Reps

TR $82-514$

August 1982

This work was supported in part by the National Science Foundation under grants MCS80-04218 and MCS82-02677. 
GENERATING LANGUAGE-BASED ENVIRONMENTS

\author{
A Thesis \\ Presented to the Faculty of the Graduate School \\ of Cornel1 University \\ in Partial Fulfillment for the Degree of \\ Doct or of Philosophy
}

by

Thomas William Reps

August 1982 
(C) Thomas William Reps 1982

ALL RIGHTS RESERVED 


\section{GENERATING LANGUAGE-BASED ENVIRONMENTS}

\section{Thomas William Reps, Ph.D.}

Cornell University 1982

This thesis concerns the design of interactive, language-based programming environments that use knowledge of a programming language to provide functions based on the structure and meaning of programs. The goal of the research is a system-constructor to enable editors for different languages to be created easily.

The most challenging aspect of such a system is the design of the semantic component, because a language-based editor performs static semantic analysis when a program is altered in order to detect erroneous constructions or to prevent illegal modifications. For efficiency, this should be performed incrementally, re-using as much old information as possible; therefore, a major focus of my research concerns a model of editing for which it is possible to perform incremental semantic analysis efficiently.

In this model, a program is represented as an attributed tree in which all attributes have consistent values; programs are modified by tree operations such as pruning, grafting, and deriving. After each modification, some of the attributes require new values; incremental semantic analysis is performed by updating attribute values to again make them all consistent. The thesis presents several algorithms for this process that are asymptotically optimal in time.

The chief disadvantage of attribute grammars is that they use large amounts of storage. The thesis discusses three aspects of utilizing 
storage efficiently in such systems. One way to reduce the amount of storage used is to reduce the number of attribute values retained at any stage of attribute evaluation. The thesis establishes two results concerning this idea: it presents one algorithm for evaluating an nattribute tree that never saves more than $O(\sqrt{n})$ attribute values, and it presents a second algorithm that never saves more than $O(\log n)$ attribute values. A second method for reducing the amount of storage is to share the space used for storing attributes whose values are complex data structures; the thesis presents a very general method for such sharing that can be applied to attributes of many types. Finally, the thesis describes how, by restricting the class of attribute grammars, it is possible to reduce the amount of storage overhead required for updating trees in optimal time. 


\section{BIOGRAPHICAL SKETCH}

Thomas William Reps was born May 28, 1956 in Ithaca, New York. He graduated from Ithaca High School in June 1973, and received the degree of Bachelor of Arts in Applied Mathematics from Harvard College in June 1977. graduating cum laude. During the year 1978-79, he was the principal implementor of the Cornell Program Synthesizer. In January 1982, he received the degree of Master of Science in Computer Science from Cornell University. He has accepted a position as a Post-Doctoral Associate with Cornel1's Department of Computer Science, and will be spending the year 1982-83 at the Institut National de Recherche en Informatique et en Automatique in Rocquencourt, France. 
For my friend and advisor, Tim Teitelbaum. 


\section{ACKNOWLEDGEMENTS}

First, and foremost, I would like to thank Tim Teitelbaum. Tim has worked very closely with me on the material in the thesis, and his guidance has been invaluable. He also extensively reviewed the thesis, and suggested many improvements. I am very lucky to have been able to work with him during the past four years.

I am also deeply indebted to Alan Demers. I have sought out Alan dozens of times when I was thoroughly confused, and almost invariably he straightened me out. I do not believe this work would have been possible without Alan's insights; each chapter of this thesis contains several of his ideas.

I would also like to thank Susan Horwitz. Her love and encouragement have been unfailing, especially during these last few months of writing. Susan has usually been the first person to hear my ideas and to read what I have written; she has been of immense help in making the ideas more precise, and the writing more succinct.

I received helpful suggestions from several people who ventured to build editors with the prototype Synthesizer Generator, including Bowen Alpern, Joe Bates, Michael Fingerhut, and Rick Palmer. Suggestions from several other individuals, including Dean Kraf $t$, David Gries, Mark Horton, Jan Prins, and Barry Rosen, were also very helpful. 
Special thanks go to Richard Conway, who, when he hired me four years ago, started a remarkable chain of events that has completely changed my life.

The National Science Foundation has provided financial support under grants MCS80-04218 and MCS82-02677.

Fina11y, my parents, Constance and John Reps, have encouraged my endeavors during both high points and low points. 
TABLE OF CONTENTS

Chapter 1: Introduction $\ldots \ldots \ldots \ldots \ldots \ldots \ldots \ldots \ldots \ldots \ldots \ldots \ldots \ldots \ldots \ldots \ldots$

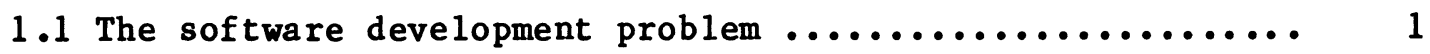

1.2 The Synthesizer Generator $\ldots \ldots \ldots \ldots \ldots \ldots \ldots \ldots \ldots \ldots \ldots \ldots . \ldots 2$

1.3 An overview of the dissertation $\ldots \ldots \ldots \ldots \ldots \ldots \ldots \ldots \ldots . \ldots 4$

Chapter 2: Providing better programming environments .......... 8

2.1 Language-based programming environments $\ldots \ldots \ldots \ldots \ldots \ldots \ldots$

2.2 Integrated program-development tools $\ldots \ldots \ldots \ldots \ldots \ldots \ldots \ldots$......... 10

2.3 Generating language-based editors $\ldots \ldots \ldots \ldots \ldots \ldots \ldots \ldots$

Chapter 3: Attribute grammars and attribute evaluation ......... 22

3.1 Attribute grammars $\ldots \ldots \ldots \ldots \ldots \ldots \ldots \ldots \ldots \ldots \ldots \ldots \ldots \ldots . \ldots \ldots$

3.2 Specifying languages with attribute grammars ......... 26

3.3 Attribute evaluation $\ldots \ldots \ldots \ldots \ldots \ldots \ldots \ldots \ldots \ldots \ldots \ldots \ldots \ldots$

Chapter 4: Incremental attribute evaluation ............... 34

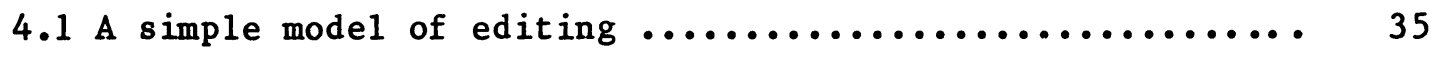

4.2 Change propagation $\ldots \ldots \ldots \ldots \ldots \ldots \ldots \ldots \ldots \ldots \ldots \ldots \ldots . \ldots \ldots$

4.3 Two-phase incremental evaluation ................ 42

4.4 Discussion $\ldots \ldots \ldots \ldots \ldots \ldots \ldots \ldots \ldots \ldots \ldots \ldots \ldots \ldots \ldots \ldots \ldots . \ldots \ldots$ 
Chapter 5: 0ptima1-time change propagation $\ldots \ldots \ldots \ldots \ldots \ldots \ldots \ldots \ldots 45$

5.1 Topological evaluation ...................... 45

5.2 Getting started $\ldots \ldots \ldots \ldots \ldots \ldots \ldots \ldots \ldots \ldots \ldots \ldots \ldots \ldots \ldots . \ldots 48$

5.3 The updating process $\ldots \ldots \ldots \ldots \ldots \ldots \ldots \ldots \ldots \ldots \ldots \ldots \ldots . \ldots . \ldots$

5.4 An optimal-time change-propagation algorithm ......... 52

5.5 Characteristic graphs, cursor motion, and subtree

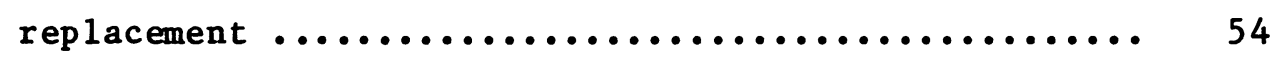

5.6 Correcting a shortcoming $\ldots \ldots \ldots \ldots \ldots \ldots \ldots \ldots \ldots \ldots \ldots \ldots$

5.7 summary $\ldots \ldots \ldots \ldots \ldots \ldots \ldots \ldots \ldots \ldots \ldots \ldots \ldots \ldots \ldots \ldots \ldots \ldots \ldots$

Chapter 6: Extending the model of editing $\ldots \ldots \ldots \ldots \ldots \ldots \ldots \ldots \ldots$

6.1 Demand attributes $\ldots \ldots \ldots \ldots \ldots \ldots \ldots \ldots \ldots \ldots \ldots \ldots \ldots \ldots . \ldots \ldots$

6.2 Operations other than subtree replacement ............ 64

Chapter 7 : Space efficient attribute evaluation $\ldots \ldots \ldots \ldots \ldots \ldots$....... 67

7.1 Motivation $\ldots \ldots \ldots \ldots \ldots \ldots \ldots \ldots \ldots \ldots \ldots \ldots \ldots \ldots \ldots \ldots . \ldots \ldots$

7.2 An evaluation algorithm that saves at most

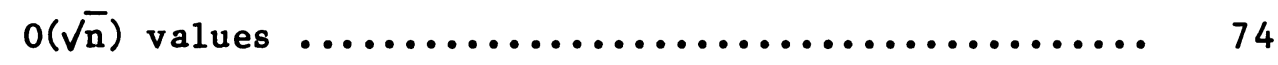

7.3 An evaluation algorithm that saves at most

$0(\log n)$ values $\ldots \ldots \ldots \ldots \ldots \ldots \ldots \ldots \ldots \ldots \ldots \ldots \ldots$

Chapter 8: Efficient representations of large attributes ....... 102

8.1 Sharing common substructures $\ldots \ldots \ldots \ldots \ldots \ldots \ldots \ldots \ldots \ldots \ldots . \ldots 102$

8.2 Rapid testing of equality $\ldots \ldots \ldots \ldots \ldots \ldots \ldots \ldots \ldots \ldots \ldots . \ldots . \ldots 108$

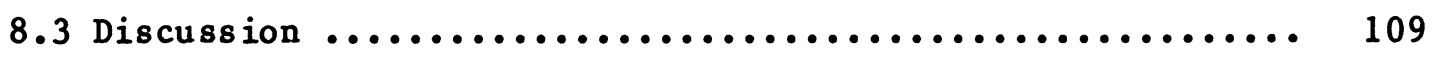


Chapter 9: Incremental evaluation for restricted classes

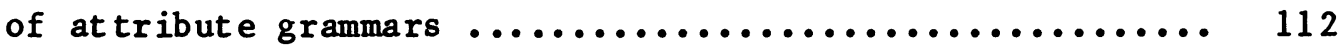

9.1 Incremental evaluation for two simple classes ......... 113

9.2 Incremental evaluation for absolutely noncircular

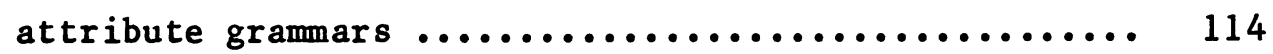

9.3 Incremental evaluation for ordered at tribute

grammars $\ldots \ldots \ldots \ldots \ldots \ldots \ldots \ldots \ldots \ldots \ldots \ldots \ldots \ldots \ldots \ldots \ldots \ldots \ldots \ldots$

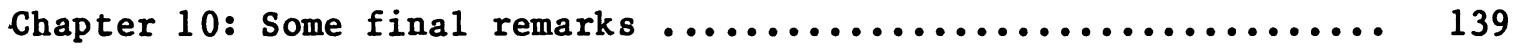

10.1 Summary of major results $\ldots \ldots \ldots \ldots \ldots \ldots \ldots \ldots \ldots \ldots \ldots . \ldots$

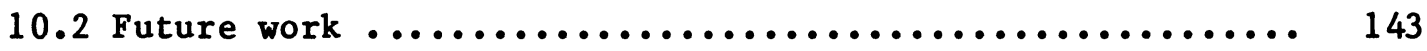

Appendix: The Synthesizer Generator Reference Manual .......... 145

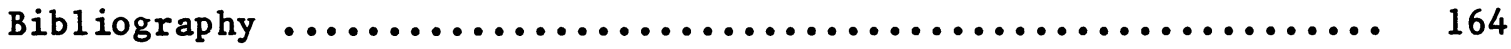

Index of symbols $\ldots \ldots \ldots \ldots \ldots \ldots \ldots \ldots \ldots \ldots \ldots \ldots \ldots \ldots \ldots \ldots \ldots \ldots$

Index of definitions $\ldots \ldots \ldots \ldots \ldots \ldots \ldots \ldots \ldots \ldots \ldots \ldots \ldots \ldots \ldots \ldots$ 
CHAPTER 1

Introduction

\subsection{The software development problem}

Software development involves massive amounts of organizational detail, only a small portion of which is intellectually important. Shielding programmers from the morass of mundane detail has been a challenge ever since computers came into existence, and a wide variety of developments in computer science can be viewed as a response to the problems encountered. For example, innovations in programing languages, such as high-level control structures, data-abstraction mechanisms, and very-high-level languages, simplify programming by hiding unimportant levels of detail. Methodologies for program development, such as step-wise refinement, formal reasoning about predicate transformers, and correctness-preserving transformations, provide guidelines for organizing the development process. Programming tools, such as editors, compilers, scanner generators, and parser generators, furnish programmers with a modest amount of automated assistance.

Despite these developments, most people involved in developing software find the current situation frustrating, for programs invariably contain bugs, and three quarters of a system's cost goes towards maintenance. Moreover, we can anticipate even more difficulties in the future as ever-more complex projects are attempted. 
A conceptual breakthrough that solves all the problems involved in software development is unlikely; however, it is a near certainty that hardware costs will continue to drop, making it possible to place increasingly sophisticated equipment at the disposal of programmers. Equipment such as single-user work-stations with large amounts of memory and high-speed video displays can be used to provide more sophisticated support for software development than has been available to date. Finding the most effective ways to provide such support is currently a very active area of research.

Winograd has argued that the programming tools of the future will be knowledge-based systems incorporating artificial intelligence techniques [Winograd 1975]. Without being overly pessimistic, it is safe to say that such systems are a decade away, if not two decades away. In the meantime, program development systems with more modest goals can be developed.

\subsection{The Synthesizer Generator}

This thesis concerns the design of interactive, language-based programming environments that use knowledge of a programming language to provide functions based on the structure and meaning of programs. This work began with the development of the Cornell Program Synthesizer [Teitelbaum \& Reps 1981], an interactive language-based programming environment with syntax-directed facilities to edit, execute, and debug programs. The example set by the Synthesizer and other language-based systems, such as Emily [Hansen 1971], MENTOR [Donzeau-Gouge et al. 1975], CAPS [Wilcox et al. 1976], LISPEDIT [Alberga et al. 1981], PDE1L

[Mikelsons \& Wegman 1980], and Gandalf [Medina-Mora \& Feiler 1981], 
motivated me to develop the Synthesizer Generator [Reps 1981], a tool for generating language-based editors for different languages using a specification of the syntax, the display format, and the static semantics of the language to be edited. The goal of this research has been the design and implementation of a system constructor to enable editors for different programming languages to be easily created.

The most challenging aspect of such a system is the design of the semantic component, for when a program is altered, a language-based editor carries out semantic analysis on the program, either for detecting erroneous constructions or for preventing illegal modifications. For efficiency, it is desirable to perform this analysis incrementally, reusing as much old information as possible. Therefore, the major focus of my research has been devising a model of editing for which it is possible to efficiently perform incremental semantic analysis when modifications are made to a program under development.

A second aspect of my work has been implementing a prototype Synthesizer Generator that incorporates the methods developed for performing incremental semantic analysis.

A third aspect of my work has been to investigate, with the help of several colleagues, the utility of the Synthesizer Generator, by using it to build a variety of experimental editors. The facilities of some of these editors duplicate facilities that have previously been implemented only in an ad hoc fashion in existing editors. Other editors that we built have broken new ground by incorporating facilities not found in any other system. 
As discussed further in Chapter 2, systems for generating language-based editors have been implemented previously by others; however, with these systems, to build an editor that provides facilities involving context-dependent program analysis, the editor-designer must explicitly specify a collection of imperative routines to be used for performing whatever semantic analysis is required after an editing modification.

In contrast, the specification language for the Synthesizer Generator is based on attribute grammars. An editor constructed with the Synthesizer Generator represents a program as an attributed tree; programs are modified by tree operations such as pruning, grafting, and deriving. After each modification to a program tree, some of the attributes require new values; incremental semantic analysis is performed by updating attribute values in response to modifications. Because the propagation of semantic information throughout the program is implicit in the attribute-grammar formalism, the editor-designer does not have to explicitly furnish a method for achieving a consistent state.

\subsection{An overview of the dissertation}

This dissertation discusses a framework for the semantic component of a language-based editor. Methods are described that deal with the basic problem in building a system for generating environments from attribute-grammar descriptions. Details of the prototype system are not covered in the dissertation, although a brief description of the system is included as an appendix. 
As mentioned above, and discussed further in Chapter 2, an environment to support programming in a particular language may include features that depend on the language's static semantics. The desire to capture such features within a formal framework has been the major motivation for my work.

Attribute grammars permit a language's syntax and semantics to be expressed in a modular, declarative fashion, and thus are a good basis for specifying language-based editors. Attribute grammars are formally defined in Chapter 3, which also presents two simple examples and discusses algorithms for attribute evaluation.

Chapters 4, 5, and 6 outline methods that allow the semantic component of a language-based editor to be generated from an attributegrammar description. Chapter 4 introduces a simple model of editing in which a program is represented as an attributed tree with consistent attribute values, and in which a program is modified by replacing a subtree of the program tree. A subtree replacement may leave some of the attributes of the tree with inconsistent values; consequently, we must have a way of updating the attributes of the tree to make them again have consistent values. Chapter 4 presents two algorithms for this process.

Making a tree consistent after a subtree replacement does not necessarily mean that every attribute needs a new value; certain attributes need new values, and the rest can keep their old values. We use AFFECTED to denote the collection of attributes that actually require new values for the tree to be consistent. Updating the tree requires doing at least as much work as the number of attributes in AFFECTED; 
however, membership in AFFECTED is not known a priori and can only be deduced as part of the updating process itself. The updating algorithms given in Chapter 4 have the drawback that they may consider an arbitrarily large number of attributes that are outside of AFFECTED, and thus these algorithms may perform an arbitrary amount of work beyond the minimum amount possible. Chapter 5 presents an algorithm that identifies attributes in AFFECTED and recomputes their values with a total cost that is proportional to the size of AFFECTED; consequently, this algorithm, unlike the algorithms of Chapter 4, is time-optimal.

Chapter 6 discusses two extensions to the model of editing. One extension introduces a class of demand attributes that are given values only on request. A second extension allows modification operations other than subtree replacement.

The primary disadvantage of attribute-grammar-based systems is that they use large amounts of storage. Chapters 7, 8, and 9 discuss three aspects of utilizing storage more efficiently in such systems. Chapter 7 investigates reducing the amount of storage used by reducing the maximum number of attribute values retained at any stage of evaluation. One result presented in Chapter 7 is an algorithm for evaluating a distinguished attribute of an n-attribute tree that never saves more than $O(\sqrt{n})$ attribute values at any stage of evaluation. A second result presented in Chapter 7 is an evaluation algorithm that never saves more than $O(\log n)$ attribute values at any stage of evaluation.

Chapter 8 describes methods for sharing space when attribute values are large data structures such as symbol tables, and presents a very 
general method for sharing storage among attributes whose values are complex data structures.

Chapter 9 returns to the subject of incremental attribute evaluation, and discusses storage problems in the context of attribute updating. The optimal-time, attribute-updating algorithm of Chapter 5 requires keeping a significant amount of information at each node of a program tree. Chapter 9 discusses other optimal-time, attributeupdating algorithms that have a much lower storage overhead than the algorithm of Chapter 5. It should be noted that the method presented in Chapter 5 applies to arbitrary noncircular attribute grammars, whereas the four methods presented in Chapter 9 apply only to subclasses of the noncircular grammars; in particular, updating methods are described for L-attributed, bottom-up/top-down, absolutely noncircular, and ordered attribute grammars.

Chapter 10 presents a summary of the ideas discussed in the thesis, draws some conclusions, and suggests some areas for further research.

As an aid to the reader, an index of symbols and an index of definitions are included at the end of the thesis. 
CHAPTER 2

Providing Better Programming Environments

A programming environment assists the programmer with the task of developing a running program from either a formal or an informal specification of its behavior. Most conventional programming environments deal with this problem by following the tool-kit paradigm; for each task that is part of program development, they provide a separate tool. For example, a minimal programming environment must at least have facilities for program entry, translation, and debugging; its tool-kit would consist of an editor, a compiler, and a debugger.

The premise of my work is that programming environments can be significantly improved by replacing the conventional collection of independent, unrelated tools by a system in which the various programdevelopment facilities cooperate effectively. This chapter discusses previous work that relates to building such a system and motivates the approach taken in this thesis.

\subsection{Language-based programming environments}

The first step in the direction of providing better programming environments is to have language-based tools, that is, tools that use knowledge of a programming language's structure to provide special program-development facilities. For example, language-based editors are 
tailored for editing objects of a particular language, and they can provide special facilities based on a program's structure, such as furnishing immediate feedback about syntax errors in a program as it is being developed [Hansen 1971]. In contrast, conventional text editors can be used to create and modify objects of many different classes, including programs, documents, and data files, but because their basic primitives are for the manipulation of unstructured text, even extensible text editors such as EMACS [Stallman 1981] and Z [Wood 1981], provide nothing more than typographical assistance.

Additional language-based tools have been developed as part of the MENTOR programming environment for PASCAL [Donzeau-Gouge et al. 1975 , 1980]. MENTOR is a general tool for manipulating trees. The core of the system is a tree-manipulation language, called MENTOL. In MENTORPASCAL, programs are represented by derivation trees of the abstract syntax of PASCAL and edited by invoking MENTOL primitives to restructure the program tree. Compositions of tree-manipulation operations can be encapsulated in MENTOL procedures; such procedures are used to extend the command language of the editor and to extend the programming environment's tool-kit.

Like conventional environments MENTOR-PASCAL follows the tool-kit paradigm; unlike conventional environments the tools of MENTOR-PASCAL take advantage of the structure of PASCAL. The philosophy of MENTORPASCAL

- . is to build specialized interpreters that help the programmer by doing various computations and rearrangements on his programs. All these interpreters communicate, between themselves as well as with the user, through the abstract syntax of PASCAL and its annotations. The development of a program is 
conceived as a multi-pass activity, each processor using as assumptions the normalization and computations effected by the previous passes.

[Donzeau-Gouge et al. 1980]

MENTOL procedures for performing scope-checking, type-checking, and data-flow analysis on PASCAL abstract-syntax trees are part of MENTORPASCAL •

These same ideas can be used in tools for verifying programs and for optimizing programs by semantics-preserving transformations:

A more formal approach [to program correctness] would request the user to state formal specifications, such as first-order assertions, and check the adequacy of the program with respect to its specifications. For instance, verification conditions may be generated through symbolic execution, and then input to a theorem prover. The formulas generated, as well as the proof trees, would of course be in turn [abstract syntax trees] manipulable by MENTOR; the user could therefore monitor the proof with the same tools he is using for manipulating his programs. - - A similar scenario can easily be designed for program optimization: local optimizations are performed by program transformations, then more global optimizations are effected at the source level after doing the necessary computations by MENTOL procedures. The program is then compiled in an object code which has its own abstract syntax. Final optimizations are performed by transformations on the object code.

[Donzeau-Gouge et al. 1980]

Such language-based tools enhance the user's power by providing a unified structural approach to manipulating objects during program development.

\subsection{Integrated program-development tools}

One drawback to the tool-kit approach is that, generally, the tool for one task cannot be applied until after certain other tasks have been completed. For instance, a program cannot be executed and debugged when it is incomplete or when it contains syntactic or static-semantic 
errors. A second drawback to the tool-kit approach is that when a program is modified, the tools must be reapplied in sequence. For example, when a programmer is debugging a program and finds an error, he must first use the editor to correct the error, then invoke the compiler for parsing, static-semantic checking, and translation, then reload the program, and on 1 y then can he resume searching for other bugs.

Two design decisions are at the root of these problems. First, tools are not usually designed to be used on incomplete programs. Second, they are not designed to make use of unchanged information from previous applications of the tool, and so after a program is modified, processing must start over again from the beginning.

By making it possible to carry out incremental semantic processing on incomplete programs, it becomes feasible to have the editor apply the tools automatically. This not only relieves the user from having to apply them manually, but it allows the facilities of the environment to be more closely integrated. For instance, incremental translation can be used to allow execution and debugging to be interleaved with editing. In the Cornell Program Synthesizer, code generation is carried out incrementally, and execution may be initiated at any stage of program development; whenever execution is suspended, for example because of a missing program element, control returns to the editor. After most editing changes to a program, it is possible to resume execution, although certain changes, such as modifying a declaration, destroy the possibility of resuming execution.

When semantic processing is carried out automatically, the editor becomes the cornerstone of the programming environment, for it provides 
not only the means for modifying a program but the means for viewing the results of program analysis. The subject of the thesis is a mechanism for incremental semantic processing that can be used to incorporate translation and analysis tools into language-based editors. It is in this limited sense that the thesis concerns programming environments; it is concerned with the static facilities of an environment, and does not discuss the run-time environment at all.

Existing language-based editors have demonstrated the usefulness of providing programmers with feedback about syntax errors as a program is being developed, but there are many applications in language-based editors for using incremental semantic analysis to provide feedback about other sorts of errors as a program is being developed. The rest of this section discusses three such applications.

\subsubsection{Enforcing language constraints}

One way of providing feedback about mistakes in a program under development is to use semantic processing to detect violations of language constraints that require widely-separated parts of a program to be interrelated. Many languages have such constraints; for instance, block-structured languages require that any variable name used must be declared in an enclosing block. To detect violations of such constraints, it is necessary to analyze the context given by the rest of the program. Whereas in a conventional programing environment a programmer will not be told of such context-dependent errors until the program is compiled, in a language-based environment the programmer can be informed immediately, while the program is still being developed. 
In the Cornell Program Synthesizer, undeclared variables are highlighted on the screen until they are declared to remind the user visually about which parts of the program contain errors. To illustrate this, consider the problem of changing the type of a program variable. One way to do this is to delete the old declaration and insert a new one; however, deleting the declaration introduces undeclared variable errors in every phrase referencing the variable. The moment a declaration is deleted, all phrases containing the undeclared variable are highlighted; when the new declaration is inserted, all are redisplayed in the normal font. For example, consider what happens when the declaration of the variable temp is deleted from the following code fragment.

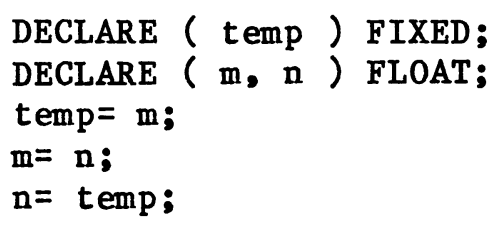

Errors are introduced in the first and third assignment statements because they use temp, so they are highlighted. 1

DECLARE ( $m, n)$ FLOAT;

$\operatorname{temp}=m_{\mathbf{g}}$

$\mathrm{m}=\mathrm{n}$;

$n=$ temp;

${ }^{1}$ In this example and the ones that follow, erroneous statements are flagged by highlighting the statement in the program display; of course, this mechanism could be replaced by, or used in conjunction with, a mechanism to provide diagnostic messages. 
When temp is redeclared, uses of temp become correct and are again displayed in the normal font.

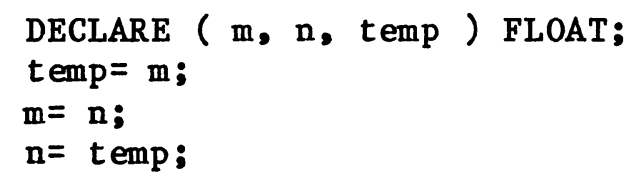

In general, detecting violations of language constraints requires widespread analysis, because modifying one part of a program may introduce an error in some other part of the program and simultaneously correct an error in a third part of the program.

\subsubsection{Detecting anomalies}

A second way of providing feedback about mistakes in a program under development is to gather and analyze information about the program's possible execution behavior without actually running the program, by means of global data-flow analysis. Although these techniques have some inherent limitations because the runtime properties of a program may require exponential time to compute, may depend on knowing properties of the input, or may be undecidable, even in these cases dataflow analysis can provide partial information. Information gathered from global data-flow analysis can be used to locate anomalous program constructions [Fosdick \& Osterweil 1976, Gillett 1977]. A languagebased editor can use these techniques to point out potential trouble spots during editing, as soon as they occur. 
Anomalies that can of ten be detected using flow analysis include:

- use of a variable before initialization

- computation of a value that is never used

- conditions that are always true or always false

- redundant expression computation

- use of low-level constructs to implement available high-level constructs

- inclusion in a parameter list of a variable that should be local

- modification of an input parameter

- array subscript out of bounds

- division by zero

- unreachable code

Anomalies like these often reflect logical errors, misconceptions about the programming language in use, a poor understanding of the algorithm being implemented, or poor programming style.

For example, consider changing the third statement in following code fragment to $" b:=a+y "$.
$\mathrm{a}:=\mathrm{x} \star \mathrm{y}$
$x:=x+1$
$\mathrm{b}:=\mathrm{x} * \mathrm{y}$
$\mathrm{x}:=0$

This change makes the second statement useless because the value assigned to $x$ can never be used; this condition could be signaled by highlighting the useless statement.

$$
\begin{aligned}
& a:=x * y \\
& \mathrm{a}:=\mathrm{x}+1 \\
& \mathrm{~b}:=\mathrm{a}+\mathrm{y} \\
& \mathrm{x}:=0
\end{aligned}
$$

Global data-flow analysis can also be used in a language-based environment to provide a completely different kind of facility; data- 
flow information can be used to answer queries about static program structure, as is done by the MasterScope facility of INTERLISP [Masinter 1980].

\subsubsection{Verification}

A third way of providing feedback about mistakes in a program is to check the program against a formal specification of its behavior. For example, consider the following code fragment that is meant to compute the product of $a$ and $b$ by repeated addition.

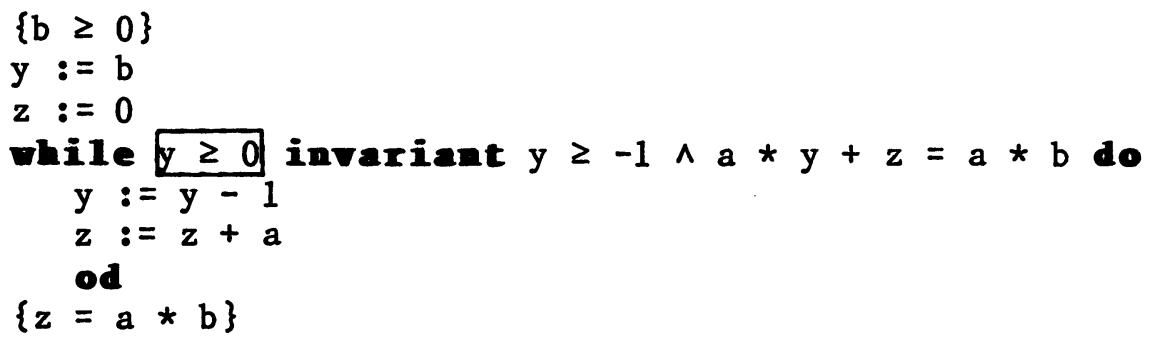

In this piece of code, the initialization of the variables $y$ and $z$ establishes the loop-invariant on entering the loop, and the loop-body reestablishes the invariant on each succeeding iteration; however, the invariant and the negation of the loop-condition fail to establish the post-condition of the loop. In the example above, this situation is signaled by highlighting the loop-condition. 
When the first clause of the invariant is changed to $y \geq 0$, the loop-body now fails to reestablish the invariant, so the statements of the body are highlighted.

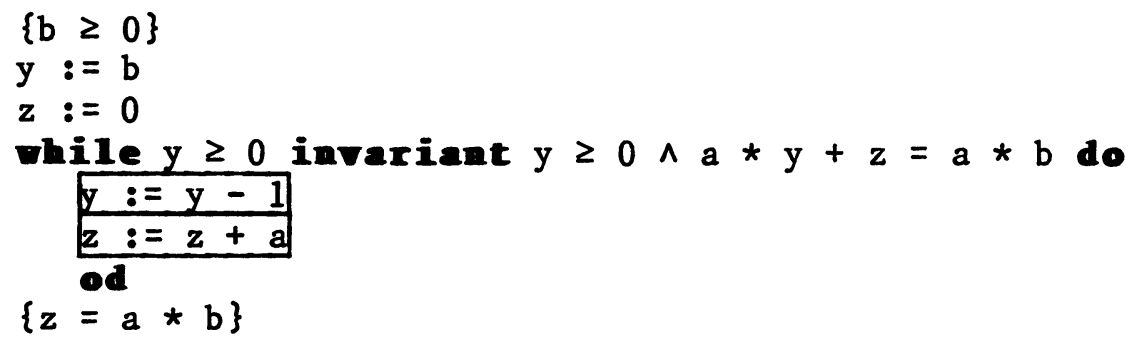

The problem is that the loop-condition allows the loop to execute one too many times. By changing the condition to $y>0$, the loop-body reestablishes the invariant, and the invariant and the negation of the condition establish the post-condition, so the entire program is displayed in the normal font.

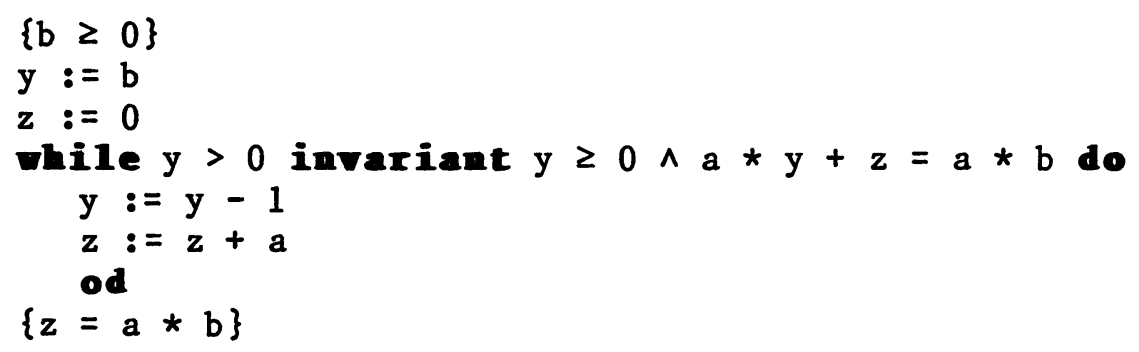

\subsection{Generating language-based editors}

Although language-based editors are tailored to particular languages, their implementations usually employ language-independent principles. Nearly all language-based editors represent programs as derivation trees of the language's abstract syntax, and the core of each system is a set of primitives for manipulating abstract-syntax trees. Exactly which operations are included among the primitives depends to a large extent on the system's user-interface. Existing language-based 
editors are either structure editors that manipulate derivation trees of a language's abstract syntax, such as Emily [Hansen 1971], MENTOR [Donzeau-Gouge et a1. 1975], and the Gandalf editor [Medina-Mora \& Notkin 1981], text editors that employ an incremental parser, such as the CAPS editor [Wilcox et al. 1976] and the PDElL editor [Mikelsons \& Wegman 1980], or hybrid editors that combine both techniques, such as the Cornell Program Synthesizer editor [Teitelbaum \& Reps 1981].

In a structure editor, editing consists of a sequence of deriving, pruning, and grafting operations interleaved with cursor movements that shift the focus of attention in the tree. An editor employing an incremental parser uses additional tree manipulations, such as split operations that break a tree up into smaller trees and join operations that assemble smaller trees into a larger tree [Morris \& Schwartz 1981, Jalili \& Gallier 1982].

The significance of organizing a language-based editor according to language-independent principles is that it allows one to build a tool for generating an editor from a language description [Hansen 1971]. Such a system takes as input a description of a language to be edited, and produces as output a language-based editor whose facilities are tailored to that language.

In its simplest form, an editor-generator uses a context-free grammar to build tables encoding the grammar in the form needed by a language-independent kernel that manipulates context-free derivation trees. However, for a generator to be capable of building an editor with facilities that require carrying out semantic processing, such as 
the ones discussed in the previous section, it must have some notion of global program analysis.

Unfortunately, the approach to this problem that has usually been taken is an ad hoc one; facilities that require global program analysis are implemented with imperative routines that walk the program tree making updates to nodes of the tree as well as to global data structures. In the Cornell Program Synthesizer, for example, when a declaration is modified, the global constraint that all uses of variables be consistent with their declarations is enforced by first voiding the entire symbol table, then reparsing all declarations to construct a new symbol table and locate multiply-declared variables, and finally reparsing all phrases to locate uses of undeclared variables and misuses of declared variables.

A similar ad hoc approach has also been taken in previous systems for generating language-based editors; they have either provided builtin mechanisms for a few special problems, such as block-structured scoping of variable names, or they have supported user-specified, semanticaction routines for making updates to the internal program representation. The first approach, which is used in the generator for Emily [Hansen 1971] and the generator for PCM [Yonke 1975], has the drawback that new applications will inevitably run up against the system's limitations. The second approach, which is used in the generator for AVID [Krafft 1981] and the generator for Gandalf [Medina-Mora \& Notkin 1981], has several drawbacks, mainly caused by the semantics being expressed imperatively, encoded in procedures with side-effects. 
The major drawback to the semantic-action approach is its imperative nature. In addition to specifying semantic actions to occur when certain elements are inserted into the program, it is also necessary to specify semantic retractions to occur when elements are deleted from the program; the latter routines must provide a method for undoing the effects of the semantic actions. In practice, there may be have to be routines for other operations besides insertion and deletion, for example, operations associated with moving the editing cursor [Medina-Mora \& Notkin 1981]. Furthermore, the editor-designer must shoulder the burden of gettıng all these procedures and their side-effects to cooperate as desired.

A second drawback to the semantic-action approach is that such a specification is not as modular as it might first appear. Though associated with a single nonterminal, each semantic routine controls a tree-walk and may depend on the structure of the entire tree; consequently, the structure of much of the grammar may have to be hard-coded into each semantic routine.

A third drawback to the semantic-action approach is that it may be difficult to write semantic routines that perform only the minimum amount of work necessary to update the internal program representation.

In contrast, this thesis discusses a framework for generating language-based editors in which the semantic component of an editor is specified formally. The syntax and semantics of the target language are specified with an attribute grammar; the editor constructed from this specification represents a program as an attributed tree. This approach avoids the complexity inherent in the semantic-action approach described 
above because of the declarative nature of attribute grammars. An attribute grammar describes relationships between attributes, and, as will be discussed in Chapters 4,5 , and 6 , the propagation of semantıc information throughout the program tree is implicit in the formalism. Because consistent relationships among the attributes can be reestablished automatically, there is no need for an explicit notion of undoing a semantic action or reversing the effect of a semantic analysis. Thus, when an editor is specified with an attribute grammar, the method for achieving a consistent state after an editıng modification is not part of the specification.

It should be noted that the advantages of attribute grammars are achieved at the expense of storage economy. The semantic-action approach maintains global data-structures that are consistent with the current syntax tree and current cursor location; the attribute-grammar approach maintains semantic values throughout the tree. Semantic retraction routines are unnecessary because attribute values are recomputed after modifications. Action routines associated with cursor motion are unnecessary because the appropriate attribute values are stored in the tree at each possible cursor location. Some approaches to making more efficient use of storage are discussed in Chapters 7, 8, and 9 . 
CHAPTER 3

Attribute Grammars and Attribute Evaluation

Attribute grammars allow the semantics of a language to be specified along with its syntax [Knuth 1968b]. Although originally employed for specifying the semantics of context-free languages, attribute grammars have since been used to express essentially the same relationships that a language-based editor needs in order to provide context-dependent facilities, including the ones discussed in the previous chapter. ${ }^{1}$ Thus, attribute grammars provide a viable basis for specifying language-based editors.

\subsection{Attribute grammars}

An attribute grammar is a context-free grammar extended by attaching attributes to the symbols of the grammar. Associated with each production of the grammar is a set of semantic equations; each equation defines one attribute as the value of a semantic function applied to other attributes in the production. The attributes of a symbol $\mathrm{X}$ are divided into two finite disjoint sets: a set of synthesized attributes, denoted $\underline{S}(X)$, and a set of inherited attributes, denoted $I(X)$. We use $A(X)$ to denote $S(X) \cup I(X)$. Each semantic equation defines a value for a synthesized attribute of the left-side nonterminal or an inherited

\footnotetext{
${ }^{1}$ See [Räihä 1980] for an extensive bibliography of the attribute grammar literature.
} 
attribute of a right-side symbol. For brevity, the arguments of the semantic function defining the value of attribute $b$ are referred to as the arguments of $b$.

A variety of methods for defining semantic translations, including model theory in logic, Montague grammars in linguistics, and denotational semantıcs in computer science, assign meanings bottom-up by defining the meaning of an object in terms of the meanings of its components. The chief attraction of such methods is their perspicuity: syntax is defined in a modular fashion by a context-free grammar; semantics is defined in an equally modular fashion on the basis of syntactic structure.

Attribute grammars are a generalization of such methods. Because inherited attributes pass information down the derivation tree at the same time that synthesized attributes pass information up the derivation tree, attribute grammars allow top-down rules to be intermixed with bottom-up rules. Despite this extension, attribute grammars retain the perspicuity of strictly bottom-up methods.

A derivation tree node $X$ defines a set of attribute instances, corresponding to the attributes of the syntactic category of $x$. Because attribute values flow in both directions, it is necessary to impose conditions to ensure that no attribute instances are defined circularly. Such a restriction is formalized using the concept of a dependency graph, a directed graph that represents functional dependencies among the attribute instances of a production $p$ or tree $T$, denoted $D(D)$ and 
$D(T)$, respectively, and defined as follows:

a) For each attribute instance b, the dependency graph contains a vertex $b^{\prime}$

b) If attribute instance b is an argument of attribute instance $c$, the dependency graph contains a directed edge $\left(b^{\prime}, c^{\prime}\right)$.

An edge from $b^{\prime}$ to $c^{\prime}$ has the meaning: $b^{\prime}$ is used to determine the value of $c^{\prime}$. An attribute grammar is noncircular when the dependency graph of every possible derivation tree is acyclic. Circularity is a decidable property of attribute grammars [Knuth 1971], but it is of inherently exponential complexity [Jazayeri et al. 1975]. One algorithm for testing circularity is given in [Knuth 1971].

Although closely related, an attribute instance $b$ in $T$ and the vertex $b^{\prime}$ in $D(T)$ are different objects. When this distinction is not made explicitly clear, the intended meaning should be clear from the context. The notation TreeNode $\left(b^{\prime}\right)$ denotes the node of $T$ with the attribute instance $b$ corresponding to $b^{\prime}$. Vertices of $D(T)$ with no incoming edges correspond to attribute instances defined by 0 -ary semantic functions, i.e. constants.

A semantic tree is a derivation tree together with an assignment of either a value or the special token null to each attribute instance of the tree. A semantic tree is fully attributed if each of its attribute instances is available, i.e. non-nul1. To further characterize 
semantic trees we introduce the notion of consistency. An attribute instance $b$ is consistent if:

a) the arguments of $b$ are available, and

b) the value of $b$ is equal to its semantic function applied to the arguments.

In all other cases, we say $b$ is inconsistent. We extend the definition of consistency to cover related concepts in semantic trees and dependency graphs; a semantic tree or a dependency graph is consistent if all of its attribute instances are consistent.

Every attribute grammar can be put into a normal form in which every semantic equation defines a value for a synthesized attribute of the left-side nonterminal or an inherited attribute of a right-side symbol, in terms of zero or more inherited attributes of the left-side nonterminal and synthesized attributes of the right-side symbols.

An attribute grammar is well-formed when the terminal symbols of the grammar have no synthesized attributes, the root symbol of the grammar has no inherited attributes, and each production includes a semantic equation for all the synthesized attributes of the left-side nonterminal and for all the inherited attributes of the right-side symbols.

The dissertation deals only with attribute grammars that are we11formed and noncircular; unless stated otherwise, we will also assume that the grammar is in normal form. 


\subsection{Specifying languages with attribute grammars}

Attribute grammars have several desirable qualities as a descriptive notation. A language is specified in a modular fashion by an attribute grammar: syntax is defined by a context-free grammar; semantics is defined in an equally modular fashion, because the arguments to each semantic function are local to one production. Propagation of attribute values through the semantic tree is not specified explicitly in an attribute grammar; rather, it is implicitly defined by the equations of the grammar and the form of the tree.

\subsubsection{Semantics of binary numerals}

As originally employed by Knuth, an attribute grammar specifies the syntax and semantics of a context-free language. The meaning of a string is defined to be the value, in some primitive domain, of a distinguished synthesized attribute of the root of the string's derivation tree; the equations of the grammar and the form of the tree implicitly define how the meaning-attribute of the root is assigned a value. For example, the grammar presented in Figure 3.1, adapted from [Knuth 1968b], illustrates an attribute grammar that defines the semantics of binary numerals.

In this grammar, all attributes have values in the domain of natural numbers. The nonterminals Number, Integer, and Bit each have a synthesized attribute, named value; Integer and Bit also have an inherited attribute, named scale. The scale attribute of a nonterminal $x$ represents the "binary place" of the rightmost bit in the subtree rooted at $X$; the value attribute of $X$ represents the value that the subtree 


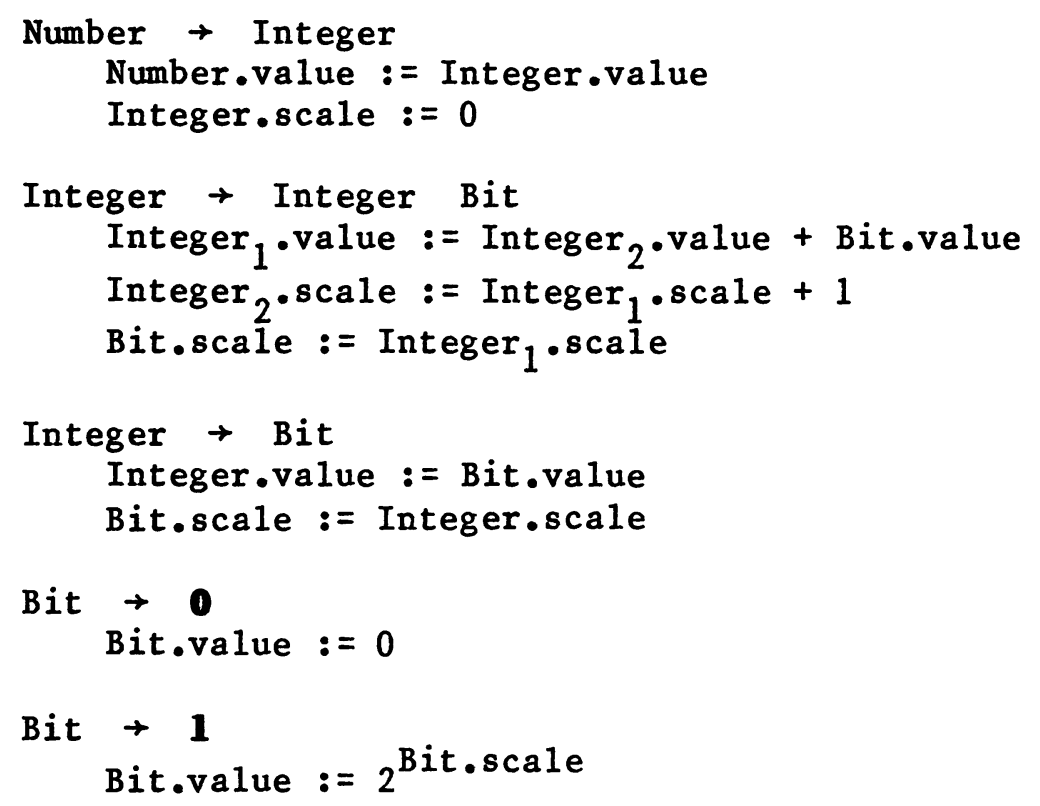

Figne 3.1. An attribute grammar that defines the semantics of binary numerals.

rooted at $X$ contributes to the total value. The meaning of a numeral is defined by the value-attribute of Number when each attribute is given a value consistent with its semantic function applied to its arguments. For example, Figure 3.2 shows how the numeral 1001 is assigned the meaning 9.

The grammar given in Figure 3.1 is only one possible way to define the meaning of binary numerals using an attribute grammar, and it is not necessarily the simplest way. Figure 3.1 demonstrates both inherited attributes and synthesized attributes; an alternative grammar defines binary numerals using only synthesized attributes [Knuth 1968b]. 


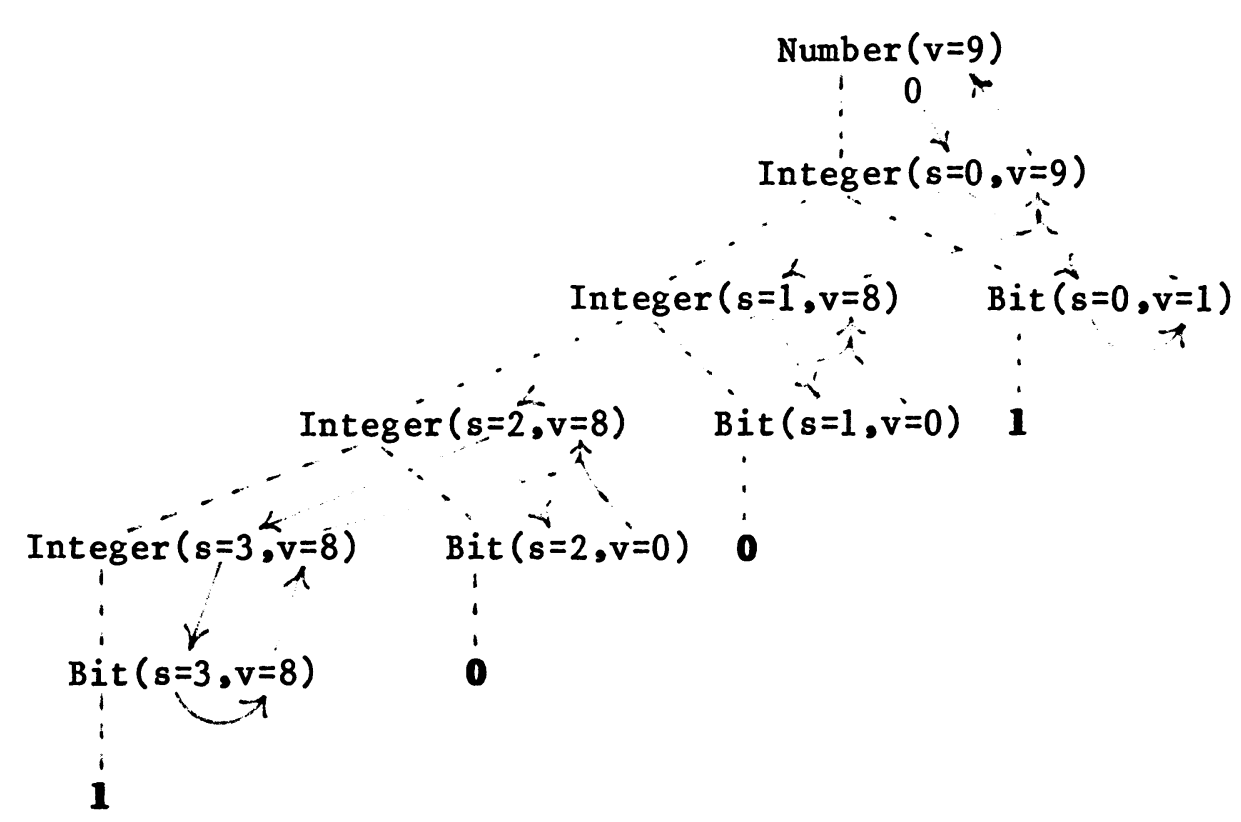

Figure 3.2. The attributed tree for the numeral 1001.

\subsubsection{Bleck-structured scoping of variable names}

In addition to being used for defining semantics, attribute grammars have been used as a framework for a number of other important programing-language problems. For example, they have been used to define translations [Lewis et al. 1974], code optimizations [Neel \& Amirchahy 1974], program correctness and correctness-preserving transformations [Gerhart 1975], data-flow analysis problems [Farrow 1977. Babich \& Jazayeri 1978], and program anomalies [Arthur \& Ramanathan]. In each of these studies, an attribute grammar is used to specify a context-dependent flow of information. To illustrate their utility for this purpose, Figure 3.3 presents an example adapted from [Bochmann 1976] that demonstrates how an attribute grammar may express 
the propagation of symbol-table information in a programming language. The underlying context-free grammar describes a simple language with

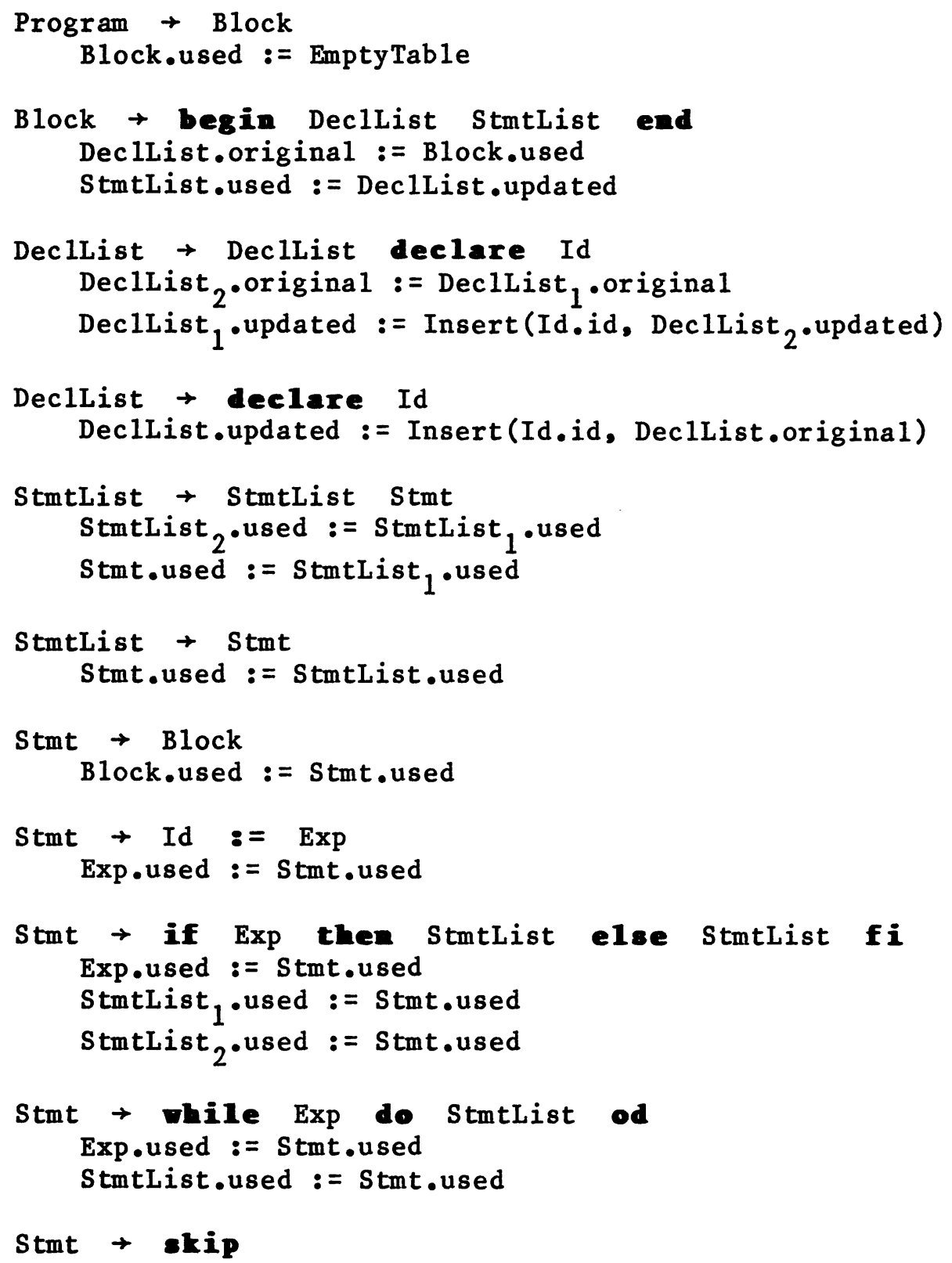

Figare 3.3. An attribute grammar that describes the propagation of symbol-table information in a simple programming language. 
blocks, declarations, conditionals, and loops. The nonterminals of the grammar are given the following attributes:

id is an synthesized attribute of Id; its value is an identifier name.

original is an inherited attribute of Decllist; its value is a partially constructed symbol table.

updated is a synthesized attribute of Decllist; its value is the original symbol table with a new entry appended.

used is an inherited attribute of Block, StmtList, Stmt, and Exp; its value is a symbol table that contains all of the variables that are legal at the nonterminal.

The attributes original and updated are threaded through the declarations to construct a symbol table from the id attributes. The symbol table is passed to every node within the scope via the used attribute. Figure 3.4 shows the semantic tree and dependency graph of the following program scheme:

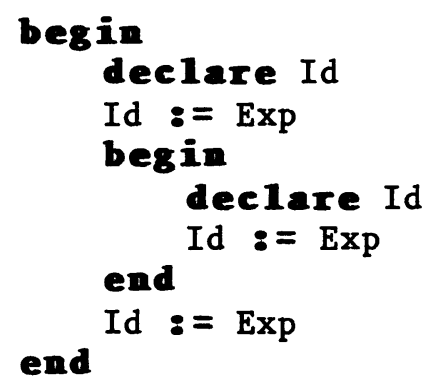

The information that is passed around the tree in this example could be used to enforce context-dependent constraints on the way names are used in a program, such as declaration-before-use and the rules for block-structured scoping of variable names. 


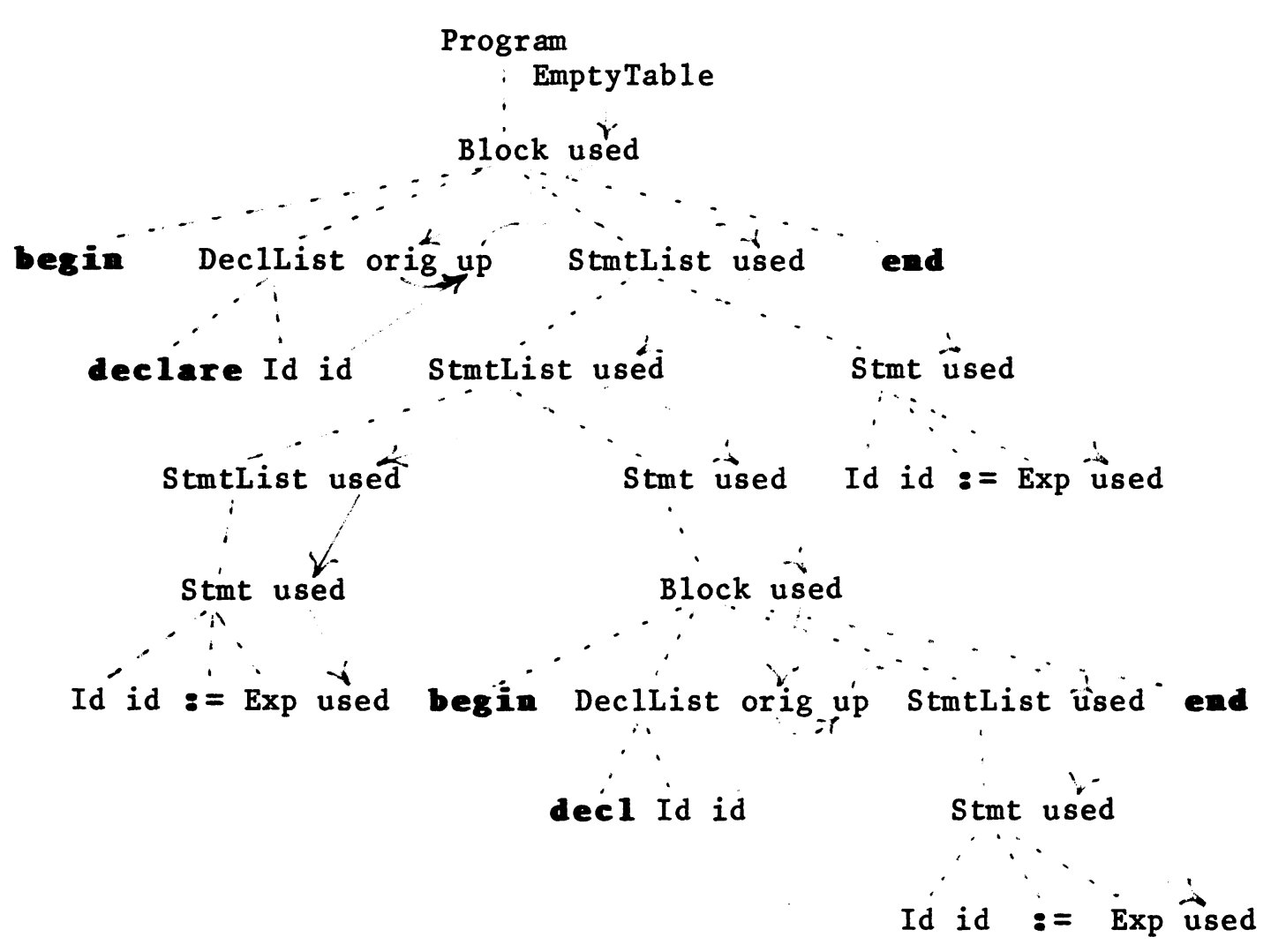

Figere 3.4. A derivation tree and its dependency graph.

The reader should not be misled by the trivial nature of the examples given above; formal definitions of programming languages of significant size have been written using attribute grammars. For instance, attribute-grammar definitions have been written for Simula [Wilner 1971], PL360 [Dreisbach 1972], Pascal [Watt 1979], and Ada [Uh1 et al. 1982].

\subsection{Attribute evaluation}

To analyze a string according to its attribute grammar specification, first construct its semantic tree with an assignment of null to 
each attribute instance, and then evaluate as many attribute instances as possible, using the appropriate semantic equation as an assignment statement. The latter process is termed attribute evaluation. The order in which attributes are evaluated is arbitrary, subject to the constraint that each semantic function be evaluated only when all of its argument attributes are available. When a1l the arguments of an unavailable attribute instance are available, we say it is ready for evaluation. Using this definition, Algorithm 3.5 states the method of [Knuth 1968b] for evaluating a semantic tree.

When EVALUATE is applied to a semantic tree $T$, its attributes are evaluated in an order that respects the partial order given by the dependency graph, $D(T)$. In practice, there must be some method for selecting the next attribute instance to evaluate; practical algorithms may be viewed as instances of EVALUATE that use different selection methods. Because each attribute instance of $T$ causes EVALUATE to perform exactly one semantic function application, we would like the total cost of this selection process to be no more than $O(|D(T)|)$.

A1gorithe 3.5. Attribute evaluation (Knuth).

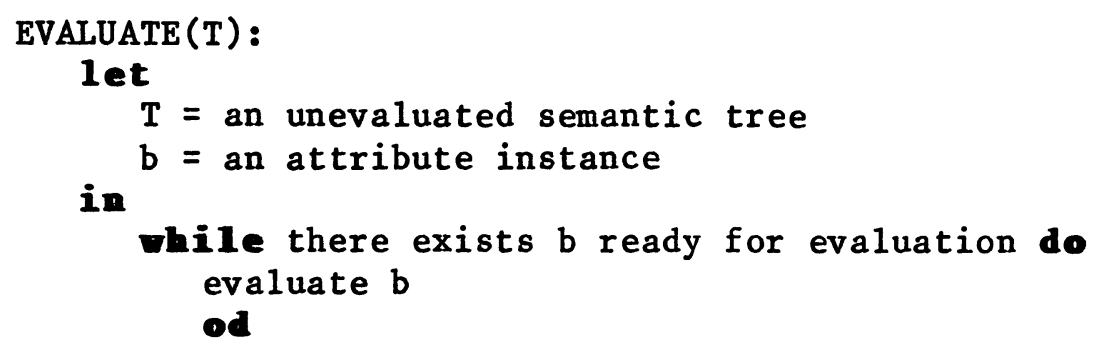


One satisfactory method is to maintain a work-1ist $S$ of all attribute instances that are ready for evaluation, as is done in Algorithm 3.6. The initial members of $S$, the attributes of $T$ that have no arguments, can be found in $O(|D(T)|)$ by traversing $T$. Set insertion and deletion can be done in unit time using standard techniques; thus, the running time of Algorithm 3.6 is $0(|D(T)|)$.

A1gorithn 3.6. Attribute evaluation.

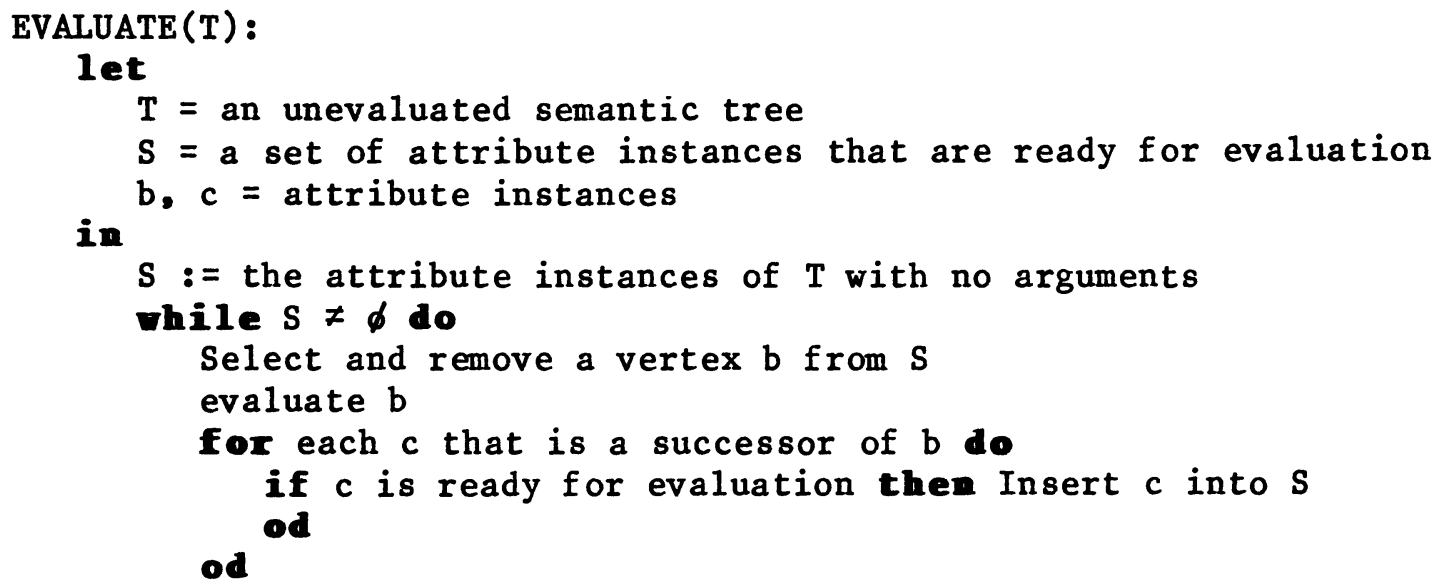


CHAPTER 4

Incremental Attribute Evaluation

\begin{abstract}
Attribute grammars have been previously used as a specification language for several compiler-writing systems, including FOLDS [Fang 1972], DELTA [Lorho 1977], MUG2 [Ganzinger et al. 1977], HLP [Räihä et al. 1978], APARSE [Milton et al. 1979], GAG [Kastens et a1.], and LINGUIST-86 [Farrow 1982b], and they have also been used as the specification language for a system that generates code-generators and codeoptimizers [Ganapathi \& Fischer 1982]. This chapter opens the discussion of how attribute grammars can be used in a system for generating language-based editors.
\end{abstract}

Both this chapter and the one following are concerned with the simple model of editing described below. The basic idea is for each editor to represent a file as an attributed tree of the attribute grammar. When editing operations modify the tree, semantic analysis is carried out by reestablishing consistent attribute values throughout the tree [Demers et al. 1981]. Attribute values indicating violation of context-sensitive language constraints may be used to annotate the program display (if only error detection is desired) or to initiate undoing the structural modification (if error prevention is desired). 
4.1. A simple model of editing

Creating a file using a language-based editor entails growing a semantic tree. During development, a file tree is a partial derivation tree; that is, it contains unexpanded nonterminals. This is potentially a problem, because at an unexpanded nonterminal $X$ we have no means for giving values to the synthesized attributes of $X$ or to any of their successors. This conflicts with our desire to maintain values for every attribute of the tree.

To avoid this problem, we require that the grammar include a completing production, $X \rightarrow 1$, for each nonterminal symbol $X$. The symbol $\perp$ denotes "unexpanded", and the semantic equations of the completing production define values for the synthesized attributes of $x$. By convention, an occurrence of an unexpanded nonterminal is considered to have derived 1. By this device, all partial derivation trees (from the user's viewpoint) are considered complete derivation trees (from the editor's viewpoint). Thus, as a program is derived, its tree may be fully attributed.

For example, for the programming language grammar given in Section 3.2.2, the completing production for Decllist can preserve the chain of symbol-table attributes by passing along the symbol table unchanged, according to the rule:

$$
\begin{aligned}
& \text { Decllist } \rightarrow \\
& \text { Decllist.updated }:=\text { Decllist.original }
\end{aligned}
$$

Modifying a program entails restructuring a derivation tree by pruning and grafting subtrees. Let $T$ be a semantic tree and $U$ be a subtree of $T$ with root node $r$ labeled $X . U$ is pruned from $T$ by removing 
the subtree rooted at $r$. Let $U^{\prime}$ be a semantic tree with root $r^{\prime}$ also labeled $X . U^{\prime}$ is grafted onto $T$ at leaf $r$ labeled $X$ by assigning the synthesized attribute values of $r$ to the synthesized attribute instances of $r^{\prime}$ and then replacing $r$ by $U^{\prime}$ in $T$. We define subtree replacement of $U$ by $U^{\prime}$ as the pruning of $U$ followed by the grafting of $U^{\prime}$ in its place. At each stage during editing, the editing cursor is positioned at an interior node of the semantic tree. An editing session is viewed as a succession of replacement operations and cursor motions starting from the complete, fully attributed, semantic tree

ROOT

with the cursor positioned at R00T. Each insertion at an unexpanded nonterminal labeled $X$ is viewed as the replacement of an instance of the completing production of $\mathrm{X}$ by a free-standing tree $U^{\prime}$ with root $\mathrm{X}$. For example, when a derivation is made according to the production $\mathrm{X} \rightarrow \mathrm{A}$ B $\mathrm{C}$, where $\mathrm{A}$ and $\mathrm{C}$ are nonterminals, $\mathrm{U}^{\prime}$ is:

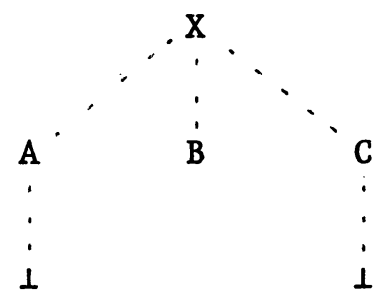

Each deletion is viewed as the replacement of a subtree U (with root $\mathrm{X}$ ) by an instance of the completing production of $\mathrm{x}$.

Because modifications may be made at any location in the program, the system must deal with free-standing trees derived from any of the nonterminals of the grammar, not just ones derived from the root symbol. 
For example, a subtree removed at $X$ becomes a free-standing tree with root $\mathrm{X}$. Such trees are retained so that they can be inserted into the program elsewhere.

The task of an incremental attribute evaluator is to produce a consistent, fully attributed tree after each subtree replacement. of course, EVALUATE could be applied to completely reevaluate the tree, but the goal is to minimize work by confining the scope of reevaluation required after each subtree replacement.

\subsection{Change propagation}

One approach to incremental attribute evaluation, called change propagation, involves propagating changes of attribute values through a fully attributed tree; throughout the process, each attribute is available, although possibly inconsistent. When the value of an attribute instance is changed to make it consistent, its successors may become inconsistent; however, if reevaluating an attribute instance yields a value equal to its old value, changes need not be propagated further. Thus, change propagation can be accomplished by using a worklist to follow attribute dependencies, as is done in Algorithm 4.1.

To do incremental attribute evaluation by Algorithm 4.1, freestanding trees must be consistent and fully attributed. In a freestanding tree $T$, the arguments of the inherited attributes of its root are not in $T$; by convention we allow inherited attributes of the root of a free-standing tree to have arbitrary values. In general, a tree resulting from grafting will not be consistent, but the inconsistencies 


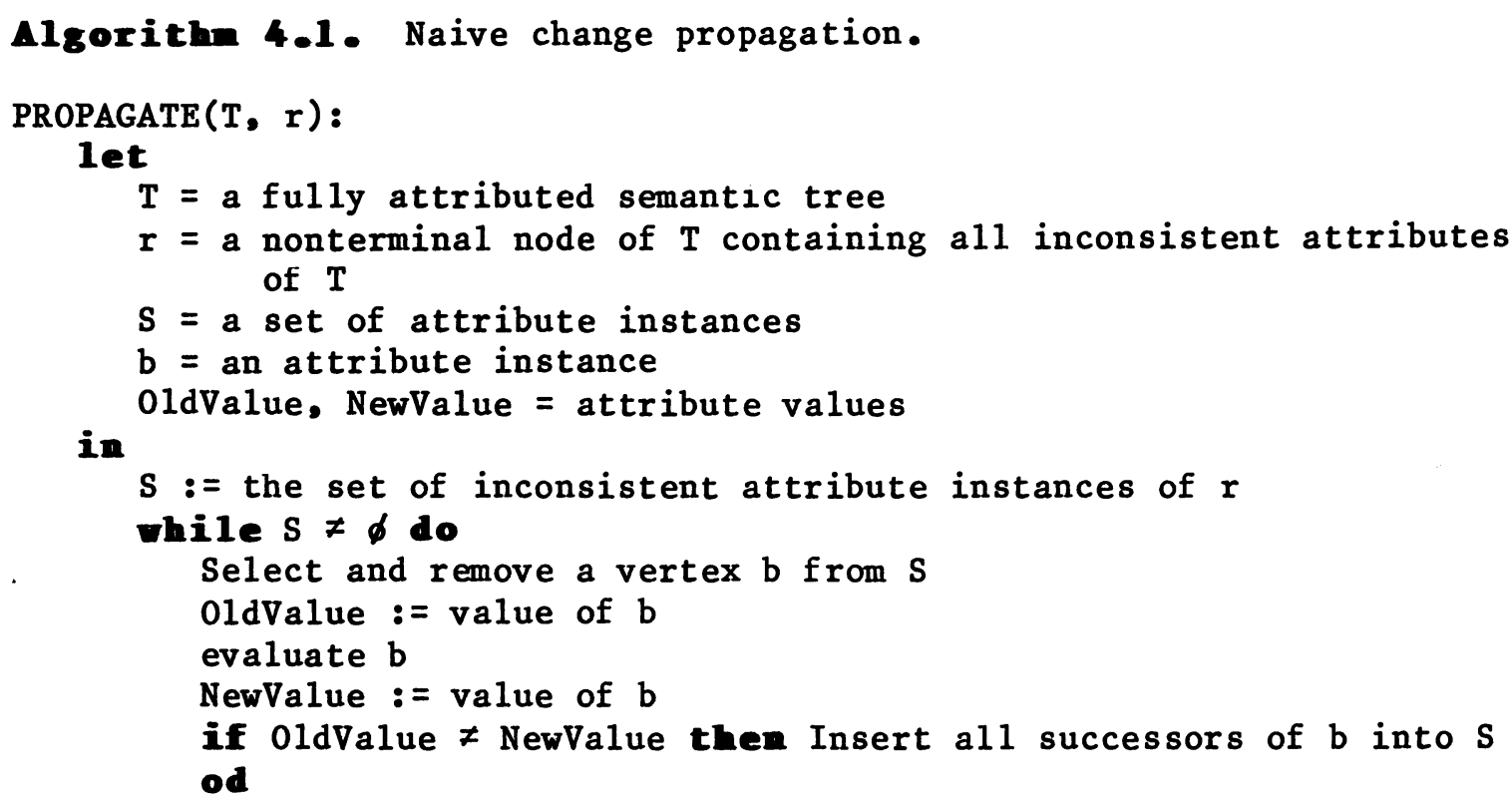

are confined to the attributes of the modification point. ${ }^{1}$ The algorithm for subtree replacement given as Algorithm 4.2 uses change propagation to reestablish consistent attribute values.

Using change propagation as part of subtree replacement makes certain editing operations inexpensive, such as inserting a subtree at a location where the attributes are identical to the attributes of the root of the subtree. Unfortunately, the behavior of change propagation is sensitive to the order in which attributes are chosen for evaluation, and if attribute dependencies are followed blindly, as in Algorithm 4.1, its behavior can be non-linear in the number of attributes reevaluated.

${ }^{1}$ If the normal form restriction is relaxed, the inconsistencies are confined to the attributes of the production that derives the modification point and the attributes of the production derived from the modification point. 


\section{Algorithe 4.2. Subtree replacement.}

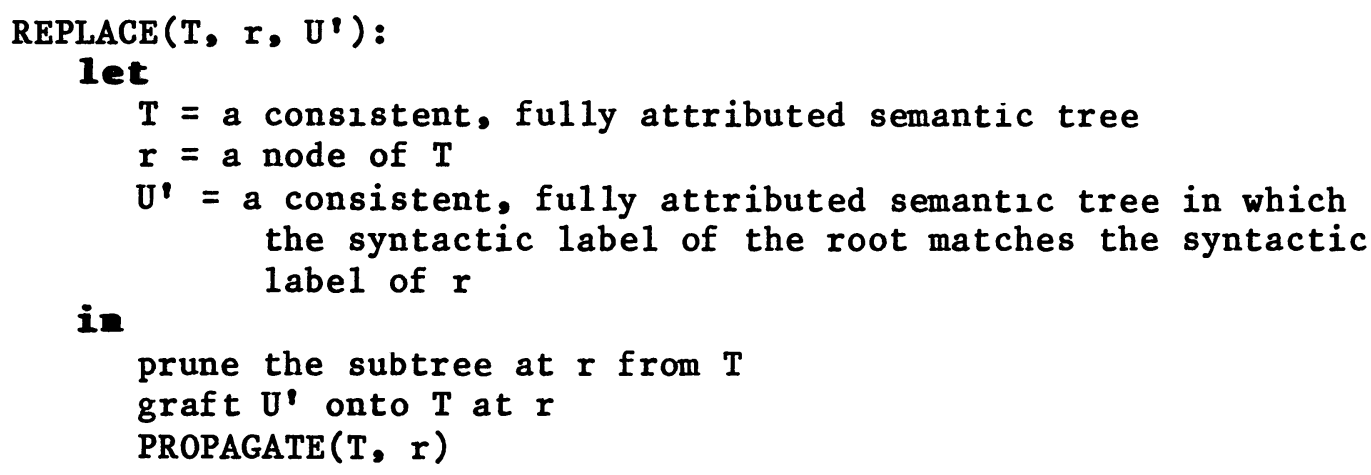

This sort of behavior occurs when updating a tree that has a dependency graph in which attributes are connected by more than one path, such as a derivation tree of the following grammar:

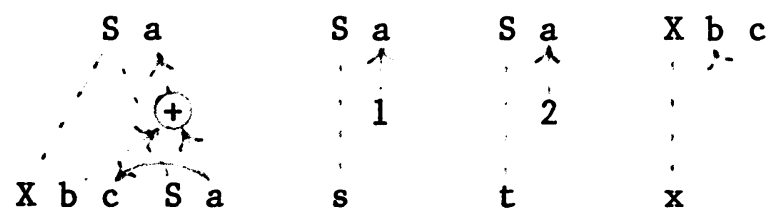

Dependency graphs of the derivation trees of this grammar have the form:

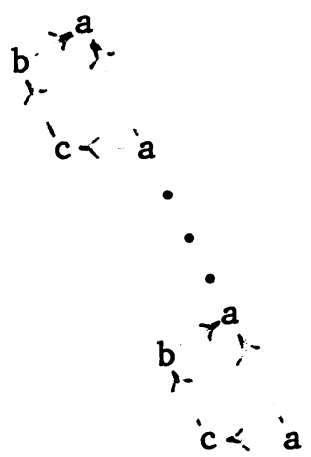

If manipulations of the work-1ist $S$ in Algorithm 4.1 obey LIFO order, i.e. $S$ is a stack, updating proceeds depth-first through the dependency graph and may take exponential time. For example, suppose we 
replace the rightmost production instance, $S \rightarrow t$, with $S \rightarrow s$, in the tree:

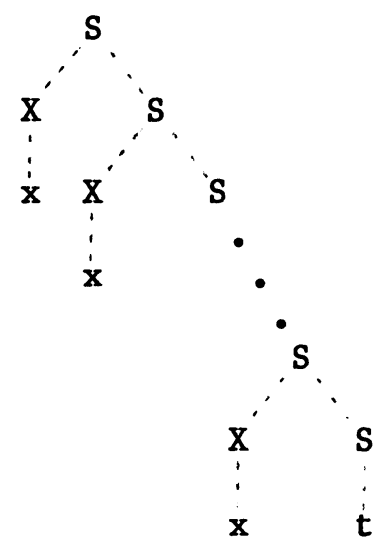

Using a depth-first strategy that always visits a vertex labeled a in preference to a vertex labeled $c$, the time required to update the tree satisfies the recurrence relation:

$$
T(h)=2 T(h-1)+\text { const }
$$

where $h$ is the height of the derivation tree; thus, $T(h)$ is exponential. Alternatively, if manipulations of the worklist $S$ obey FIFo order, i.e. S is a queue, updating proceeds breadth-first through the dependency graph and may take tıme quadratıc in the height of the tree, as seen by the following argument. Assuming a breadth-first strategy that always visits a node labeled a in preference to a node labeled $c$ is used in the example above, updating progresses through the $h / 3+1$ stages depicted in Figure 4.3, where a label $(j)$ on a node means that the node appears in the $j^{\text {th }}$ position in the queue. Getting from the $i^{\text {th }}$ stage to the $i+1^{s t}$ stage requires three complete passes through the queue, which have $\operatorname{cost} 3 i+2,3 i+3$, and $3 i+4$, so the total cost of the $h$ passes 


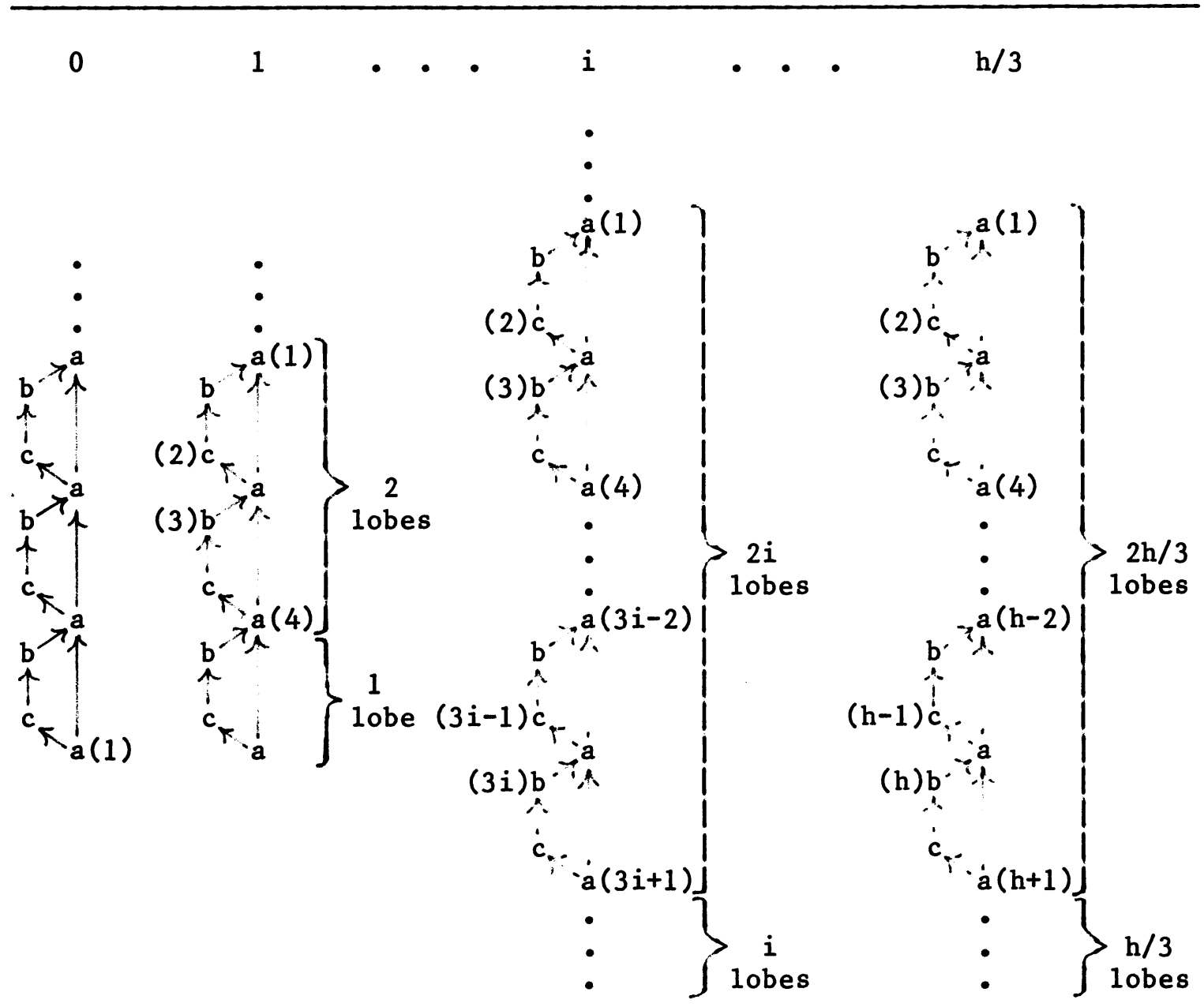

Figure 4.3. Stages of updating by a breadth-first strategy.

it takes to reach stage $\mathrm{h} / 3$ is:

$$
\sum_{i=0}^{h / 3}(9 i+9)=0\left(h^{2}\right)
$$

A similar argument about the next $2 \mathrm{~h}$ passes through the queue shows that an additional $O\left(h^{2}\right)$ steps are needed to finish updatıng the tree. 


\subsection{Two-phase incremental evaluation}

The non-linear behavior of change propagation that can result from using Algorithm 4.1 can be avoided by using an alternative method for subtree replacement, given below as Algorithm 4.4. Algorithm 4.4 uses the procedure NULLIFY to propagate the special value null to all attributes that depend on the synthesized attributes of the modification point. NULLIFY is also applied to free-standing trees that result from subtree replacements to propagate nell to all attributes that depend on the inherited attributes of the tree's root. Thus when the freestanding tree $U^{\prime}$ is grafted onto $T$, the state of the tree $T+U^{\prime}$ is a

A180rithe 4.4. Subtree replacement.

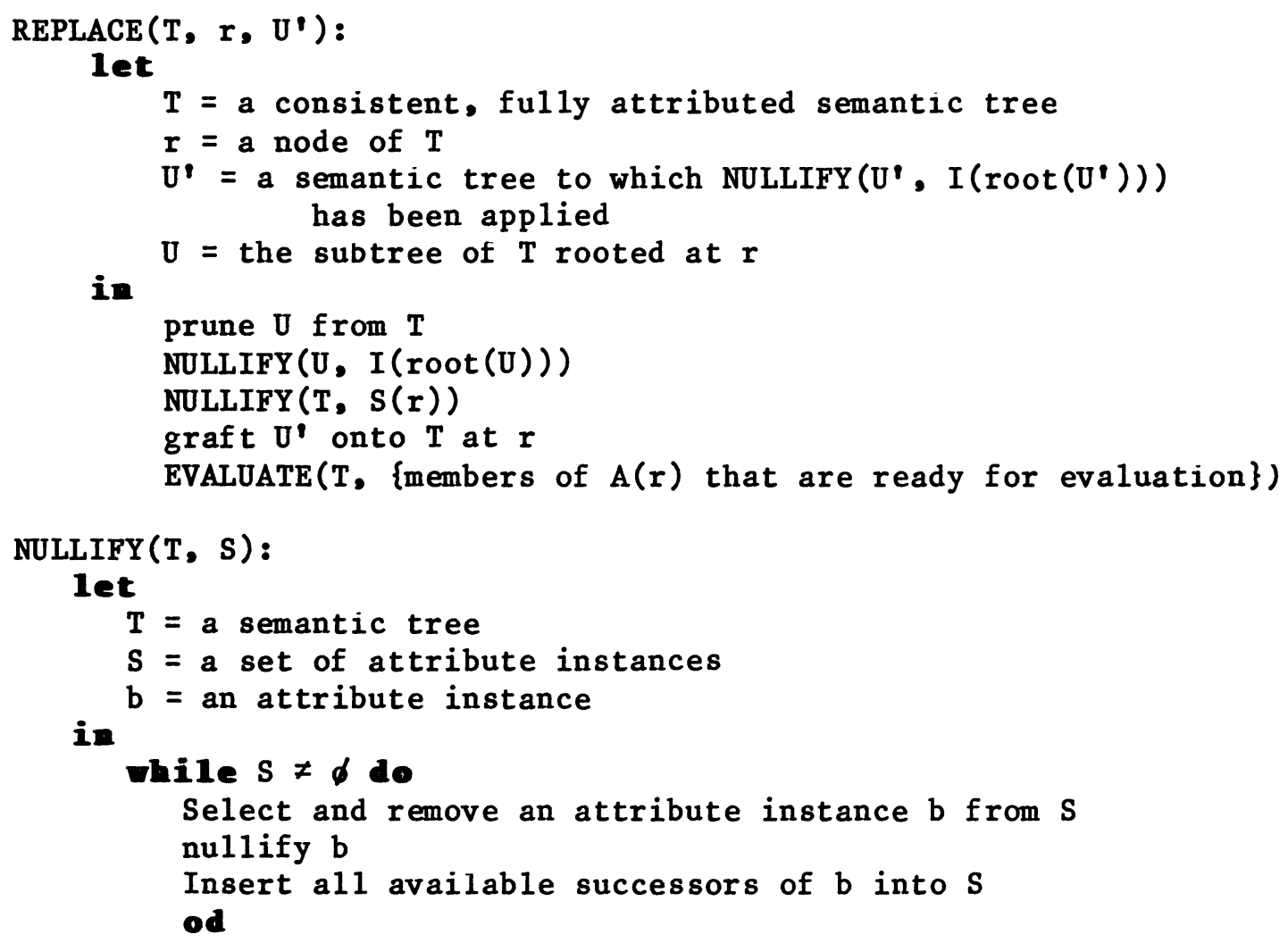


possible result of some execution sequence had evaluation started from a completely unattributed tree. Consequently, $T+U^{\prime}$ can be updated by restarting EVALUATE, passing it the attributes of $r$.

Both NULLIFY and EVALUATE have the property that they only consider an attribute once, so the total work done by Algorithm 4.4 is linear in the number of attributes considered.

\subsection{Discussion}

A derivation tree modification directly affects the values of the attributes at the point of modification. In our model of editing, attribute values must be updated in response to each modification to leave the semantic tree consistent and fully attributed. Out of the entire collection of attributes in the tree, only certain ones require new values. To be more precise, let $T^{\prime}$ denote the inconsistent tree resulting from a subtree replacement, and $T^{\prime \prime}$ denote $T^{\prime}$ after it has been updated. We define AFFECTED to be the set of attribute instances that have different values in the two trees; consequently, $O(\mid$ AFFECTED $\mid$ ) is the minimal amount of work required to update $T^{\prime}$ after subtree replacement. However, it is important to understand that AFFECTED is not known a priori, and can only be inferred from the updating process itself.

The two-step evaluator given as Algorithm 4.4, consisting of nullification followed by evaluation, is not optimal because the size of the set of all attributes that depend on attributes of the modification point is not related to the size of AFFECTED in any fixed way. Consequently, the two-step algorithm does extensive propagations even for modifications that are potentially free. 
The naive change propagation evaluator given as Algorithms 4.1 and 4.2 is very sensitive to the order in which attributes are selected as candidates for new values. With the right selections, a tree is updated optimally; however, there is no guarantee that the right selections will be made, and when attributes are selected in the wrong order, the algorithm is not only suboptımal, but the time it uses can be non-1inear in the number of attributes considered. 
CHAPTER 5

Optima1-Time Change Propagation

A change propagation evaluator will be time-optimal if the new value computed for each attribute is the attribute's final value, if the number of recomputations of each attribute is bounded by a constant, and if the bookkeeping required to select the order of recomputation is linear in the number of attributes changed. Conversely, if an attribute is ever (temporarily) reassigned a value other than its final value, spurious changes are apt to propagate arbitrarily far beyond the boundaries of AFFECTED, leading to suboptimal running time.

This chapter describes a version of the change-propagation algorithm PROPAGATE that identifies attributes in AFFECTED and computes their new, final values without ever giving an attribute a temporary value. Both the total number of semantic function applications and the total cost of bookkeeping operations are proportional to the size of AFFECTED; consequently, PROPAGATE is asymptotically optimal in time [Reps 1982].

\subsection{Topological evaluation}

Before discussing an optimal version of PROPAGATE, it is instructive to reconsider (non-incremental) attribute evaluation. We stated earlier that attributes must be evaluated in an order that respects the 
partial order given by the tree's dependency graph. Thus, attribute selection is related to the problem of topological sorting [Knuth 1968a]. To do attribute evaluation, one may perform the topological sort first and then evaluate the attribute instances in the resulting order [Lewis et a1. 1974, Kennedy \& Ramanathan 1979], or one may interleave sorting with attribute evaluation, a process we call topological evaluation.

The topological evaluation algorithm stated below as Algorithm 5.1 is essentially topological sort, except that in line ( $*$ ) attributes are evaluated instead of being assigned a topological number. Algorithm 5.1 is nearly identical to Algorithm 3.6, except that Algorithm 5.1 makes use of a graph data structure $G$, which is initially the entire dependency graph $D(T)$. The set $S$, which is initially the set of vertices of $D(T)$ with in-degree 0 , is a work-list containing the set of attributes

Algorith 5.1. Topological evaluation.

EVALUATE(T):

let

$T=$ an unevaluated semantic tree

$\mathbf{G}=$ a directed graph

$S=a$ set of attribute instances

$b, c=$ attribute instances

in

$G:=D(T)$

$S:=$ the set of vertices of $G$ with in-degree 0

while $S \neq \phi d o$

(*) evaluate b

for each $c$ that is a successor of $b$ in $G$ do

Remove (b, c) from $G$

if in-degree ${ }_{G}(c)=0$ then Insert $c$ into $S$

od

od 
that are ready for evaluation. G changes as attributes are evaluated to reflect the dependencies of unevaluated attributes only. The in-degree of each vertex retlects the number of unevaluated attributes that the vertex depends upon. Thus, an edge $(b, c)$ in $G$ indicates that attributes $b$ and $c$ are unevaluated and $c$ depends on $b$. This means that upon evaluation of an attribute $b$, all its outgoing edges $(b, c)$ are deleted from $G$, and if this action causes $c$ to have in-degree $0, c$ is inserted in the work-1ist $S$ because it is ready for evaluation.

The algorithm relies substantially on the fact that $D(T)$, and hence $G$, is acyclic. If $G$ has an edge, there must be at least one unevaluated attribute of $G$ with in-degree 0 ; thus, $S$ is not empty. Upon termination $S$ is empty, so $G$ contains no edges; therefore, all attributes have been evaluated.

The optimal-time version of PROPAGATE is most easily understood as a generalization of topological evaluation. As in topological evaluation, PROPAGATE keeps a work-list of attributes that are ready for reevaluation, and it schedules reevaluations according to an attribute's position in a graph that retlects dependencies among attributes that have not been reevaluated. What makes PROPAGATE asymptotically optimal is that the scheduling graph never grows larger than $0(|A F F E C T E D|)$; on each step, the size of the scheduling graph is proportional to the number of attributes that have received new values since updating began. Vertices of the initial scheduling graph represent just the attributes of the point of subtree replacement. Thereafter, the scheduling graph expands only when changes propagate to attributes that are arguments of attributes outside the current graph. 


\subsection{Getting started}

Let us suppose a subtree replacement takes place at node $r$. By virtue of the way attribute values are exchanged during subtree replacement, the only attributes that are inconsistent when change propagation is initiated are attributes of $r$. No progress would be made by reevaluating attributes of other nodes, so PROPAGATE should start of $f$ by reevaluating an attribute of $r$; however, we need to choose an attribute of $r$ whose arguments are guaranteed not to change value, thereby assuring that the new value computed is the correct final value.

To make the right selection, it is necessary to know about transitive dependencies among the attributes of $r$. If $b$ and $c$ are attributes of $r$, and $c$ transitively depends on $b$, then $b$ must be reevaluated before c. These relationships can be represented by a directed graph whose vertices are the attributes of $r$ and whose edges represent transitive dependencies among the attributes.

To discuss this idea more precisely, we make the following definitions:

a) Given directed graphs $A=\left(\nabla_{A}, E_{A}\right)$ and $B=\left(V_{B}, E_{B}\right)$, that may or may not be disjoint, the union of $A$ and $B$ is:

$$
A \cup B=\left(V_{A} \cup V_{B}, E_{A} \cup E_{B}\right)
$$

b) The deletion of $B$ from $A$ is:

$$
A-B=\left(V_{A}, E_{A}-E_{B}\right)
$$

Note that deletion deletes only edges. 
c) Given a directed graph $A=(V, E)$ and a set of vertices $\nabla^{\prime} \subseteq V$, the projection of $A$ onto $\nabla^{\prime}$ is:

$$
A / V^{\prime}=\left(V^{\prime}, E^{\prime}\right)
$$

where $E^{\prime}=\left\{(v, w) \mid v, w \in \nabla^{\prime}\right.$ and there exists a path from $v$ to $w$ in A that does not contain any elements of $\left.V^{\prime}\right\}$.

Transitive dependencies are represented locally by subordinate and superior characteristic graphs. We let each node $r$ in a semantic tree be labeled with its subordinate characteristic graph, denoted r..., its superior characteristic graph, denoted $\mathbf{x} \cdot \overline{\bar{c}}$, or both. The subordinate characteristic graph at node $r$ is the projection of the dependencies of the subtree rooted at $r$ onto the attributes of $r$. To form the superior characteristic graph at node $r$, we imagine that the subtree rooted at $r$ has been pruned from the semantic tree, and project the dependency graph of the remaining tree onto the attributes of $r$. Note that the vertices of the characteristic graphs at $r$ correspond to the attributes of $r$.

Formally, let $r$ be a node in semantic tree $T$, and let the subtree rooted at $r$ be denoted $T_{r}$; the subordinate and superior characteristic graphs at $\mathbf{r}$ are defined by:

$$
\begin{gathered}
r \cdot C \equiv D\left(T_{r}\right) / A(r) \\
r \cdot \bar{C} \equiv\left(D(T)-D\left(T_{r}\right)\right) / A(r)
\end{gathered}
$$

Knowing the subordinate and superior characteristic graphs at $r$ allows us to construct the graph r.c $\cup$ r. $\bar{c}$. An edge of this graph represents a transitive dependence between two attributes of $r$. An attribute in this graph that has in-edges depends on one of the other 
attributes of $r$; consequently, it is not a suitable first choice for reevaluation. An attribute with in-degree 0 in r.c $\cup$ r. $\bar{c}$ does not depend on any of the other attributes of $r$ and therefore is a suitable first choice; there is at least one such attribute because the attribute grammar is noncircular.

\subsection{The updating process}

The previous section argues that the graph r.c $\cup$ r. $\bar{C}$ must be constructed in order to choose the first attribute for reevaluation. In general, this graph represents a partial order that PROPAGATE must respect throughout the updating process; however, as updating progresses, it is necessary to know more than just the dependency relationships among the attributes of $r$. When the value of an attribute instance is changed, all attributes that use it as an argument may become inconsistent; it is necessary to take into account the dependencies that involve these attributes.

To schedule reevaluations, PROPAGATE employs a graph $\mathrm{M}$, called the model, and a set $S$, used as a work-1ist. $M$ is a generalization of the graph discussed in the previous sectıon; it represents dependencies among the attributes of a connected region of the tree, rather than just dependencies among the attributes of a single node. A vertex of $M$ corresponds to an attribute; an edge of $M$ represents a functional dependence, which may be either a direct dependence or a transitive 
dependence. In particular, M contains:

a) edges representing direct dependencies in the modeled region of the tree,

b) edges of the superior characteristıc graph of the apex of the region, and

c) edges of the subordinate characteristic graphs of the frontier of the region.

Characteristic graph edges represent transitive dependencies transmitted entirely outside the modeled region of the tree.

Initially, $M$ is the graph $r, C \cup r \cdot \bar{C}$, and $S$ is the set of vertices of $M$ with in-degree 0 . As long as $M$ covers the affected region of the tree, PROPAGATE does a topological evaluation of $M$. Every time an attribute $b$ is reevaluated, the old value and the new value are compared; if they differ, and if $b$ is an argument of an attribute instance that is outside of $M$, then $M$ is expanded by one production instance so that it includes the successors of $b$.

To describe an expansion formally, we detine the functions ExpandedSubordinate and ExpandedSuperior, which produce graphs that are refinements of a node's characteristic graphs. If node $s_{0}$ is the parent node in a production instance $p$ that consists of the nodes $\left\{s_{0}, s_{1}, \ldots, s_{k}\right\}$, we define:

$$
\text { ExpandedSubordinate }\left(s_{0}\right) \equiv D(p) \cup s_{1} \cdot C \cup \ldots \cup s_{k} \cdot C
$$


For any other node $s_{j}$ in the production instance, we define:

$$
\text { ExpandedSuperior }\left(s_{j}\right) \equiv D(p) \cup s_{0} \cdot \bar{C} \cup s_{1} \cdot C \ldots s_{j-1} \cdot C \cup s_{j+1} \bullet C \ldots s_{k} \cdot C
$$

A model is expanded by the procedure EXPAND, given in Figure 5.2. In addition to deleting a characteristic graph from the model, and unioning the model with the corresponding expanded characteristic graph, an expansion also involves making insertions into the work-1ist $S$. At the time an attribute is brought into the model, if its in-degree in the model is 0 it is inserted into the work-list because it is ready to be reevaluated. Because an expansion is limited to a single production, EXPAND has a bounded cost for a given grammar.

\subsection{An eptimal-time change-propagation algorithm}

PROPAGATE, an incremental attribute evaluator stated below as Algorithm 5.3, interleaves topological evaluation with calls to EXPAND.

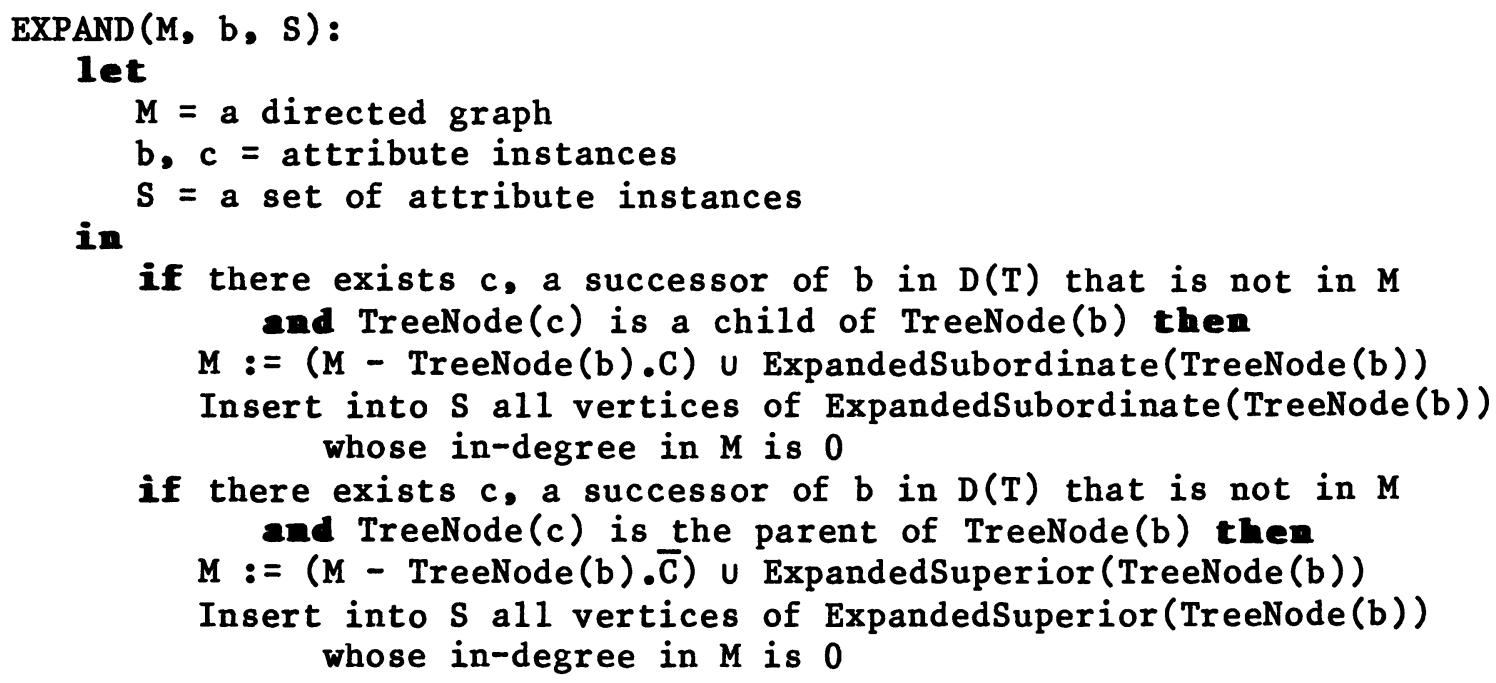

if there exists $c$, a successor of $b$ in $D(T)$ that is not in $M$ and TreeNode $(c)$ is a child of TreeNode(b) then $M:=(M-T r e e N o d e(b) . C)$ U ExpandedSubordinate(TreeNode (b)) Insert into $S$ all vertices of ExpandedSubordinate(TreeNode(b)) whose in-degree in $M$ is 0

if there exists $c$, a successor of $b$ in $D(T)$ that is not in $M$ and TreeNode $(c)$ is the parent of TreeNode(b) then $M:=(M-T r e e N o d e(b) . \bar{c}) \cup$ ExpandedSuperior (TreeNode (b)) Insert into $S$ all vertices of ExpandedSuperior(TreeNode(b)) whose in-degree in $M$ is 0

Figure 5.2. Expanding a model. 


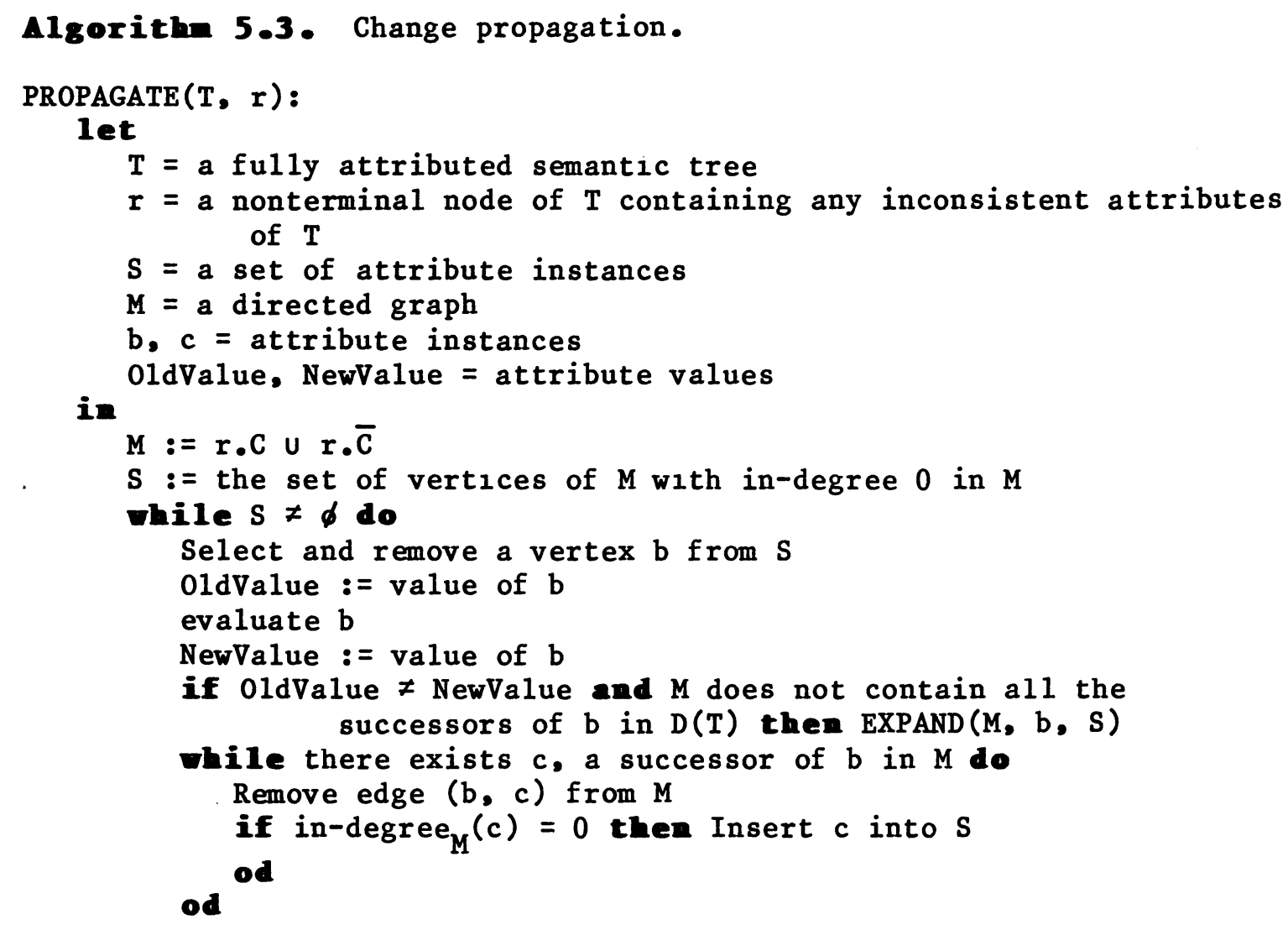

When PROPAGATE terminates, the model M consists of the attributes that are part of any production instance in which an attribute changed value. All dependency-graph edges of this region have been inserted into $M$ by the expansion process and have been removed from $M$ by the topological evaluation process.

The number of vertıces and edges introduced into $M$ by an expansion is bounded by the size of the largest production in the grammar. $M$ is only enlarged when a new member of AFFECTED has been identified; consequently, the maximum size of $M$ is $0(|A F F E C T E D|)$. The cost of considering a vertex is one semantic function application and a constant amount 
of bookkeeping work. The total number of semantic function applications and the total cost of bookkeeping operations in PROPAGATE are O(|AFFECTED|); thus, PROPAGATE is asymptotically optimal in time.

Characteristic-graph edges, representing transitive dependencies in $D(T)$, are crucial to the optimal behavior of PROPAGATE. The presence of characteristic-graph edges ensures that an attribute is never updated until all its ancestors are consistent; consequently, an attribute can never be assigned a temporary value during updating. Removing a characteristic-graph edge allows PROPAGATE to skip, in unit time, arbitrarily large sections of $D(T)$ in which values do not change.

\subsection{Characteristic graphs, cursor motion, and subtree replacement}

Until now, we have tacitly assumed that both subordinate and superior characteristic graphs were maintained at each node of the tree. However, a subtree replacement can radically alter transitive dependencies among attributes. In fact, a subtree replacement at node $\mathbf{r}$ can alter characteristic graphs arbitrarily far away from $r$; maintaining every characteristic graph in the tree would make subtree replacements too expensive.

Fortunately, PROPAGATE does not need every characteristic graph. After a subtree replacement at node $r$, PROPAGATE never needs subordinate characteristic graphs at any of the nodes on the path from $r$ to the root of the tree, and it never needs superior characteristic graphs anywhere else. PROPAGATE needs both characteristic graphs only at $r$. We say 
that $T$ is prepared for propagation at $x$ when, as in Figure 5.4,

a) $\mathbf{r}$ is labeled with both its subordinate characteristic graph, $\mathrm{r} . \mathrm{C}$, and its superior characteristic graph, r. $\bar{c}$,

b) each node $s$ on the path from $r$ to $\operatorname{root}(T)$ is labeled with its superior characteristic graph, s. $\bar{c}$, and

c) each node $t$ not on the path from $r$ to $\operatorname{root}(T)$ is labeled with its subordinate characteristic graph, t.c.

The editor maintains the invariant that the semantic tree is prepared for propagation at the position of the editing cursor. This invariant must be reestablıshed after each movement of the editing cursor to a new location. Every cursor movement can be defined as a sequence of the operations AscendToParent and DescendToChild $(j)$. Given that the editing cursor is positioned at node $r$ of $T$, and that $T$ is prepared for propagation at $r$, AscendToParent has the side effect:

$\mathbf{r} \cdot \overline{\mathrm{C}}:=\mathbf{n} \mathbf{1 1}$

parent $(r) \cdot C:=$ ExpandedSubordinate $($ parent $(r)) / A($ parent $(r))$

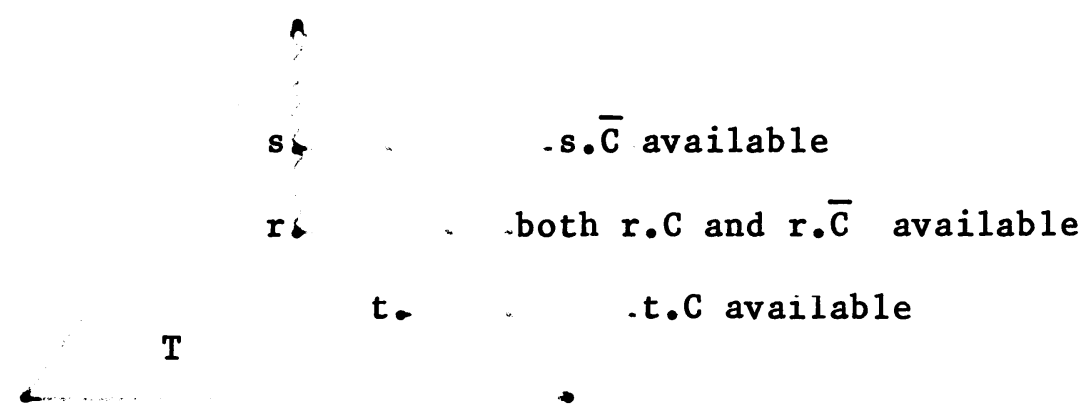

Figure 5.4. T prepared for propagation at $r$. 
DescendToChild( $j)$ has the side effect:

$$
\begin{aligned}
& r_{\bullet} \cdot C_{j}:=\operatorname{mal1} \\
& r_{j} \cdot \bar{C}:=\text { ExpandedSuperior }\left(r_{j}\right) / A\left(r_{j}\right)
\end{aligned}
$$

where $r_{j}$ denotes the $j^{\text {th }}$ child of $r$. For a given grammar, each of these updates has unit cost. A movement of the editing cursor over a path of length $m$ in the semantic tree costs $O(m)$.

The invariant that the tree is prepared for propagation at the position of the editing cursor must also be reestablished after a subtree replacement before PROPAGATE is called. By retaining subordinate characteristic graphs when a subtree is pruned, a free-standing tree is prepared for propagation at its root. After a subtree $U$ at node $r$ is replaced by a free-standing tree $U^{\prime}$ with root s, setting the superior characteristic graph at the cursor to be $r \cdot \bar{c}$ and the subordinate characteristic graph to be s.C reestablishes the invariant.

\subsection{Correcting a shortcoming}

As presented, PROPAGATE has a shortcoming: an attribute instance that becomes part of $M$ eventually gets evaluated, even if none of its arguments receives a new value. Furthermore, this can happen to an attribute instance not once, but up to three times. For example, when the cursor is positioned at $B$ in:

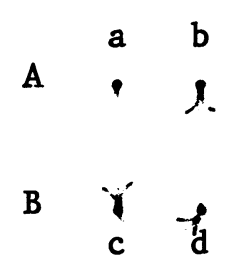


the initial configuration of $M$ is:

$$
\text { c } \quad r_{d}^{\bullet}
$$

In the following action sequence $c$ is evaluated twice:

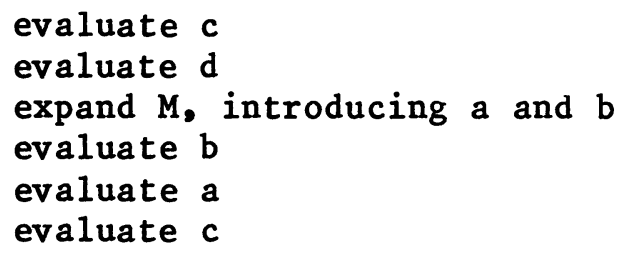

It is clear that c cannot get a new value from the second evaluation, and since evaluations may be expensive this is undesirable behavior. Note that this is not a counter-example to the optimal time bound; in general, attributes at the cursor location can be placed on the worklist (and evaluated) at most three times, while all other attributes can be placed on the worklist at most twice.

Such needless evaluations can be avoided by using an additional set, named NeedToBeEvaluated, as follows:

a) NeedToBeEvaluated is initialized to contain all the vertices of the initial model.

b) When the value of an attribute instance b is changed, every successor of $b$ is inserted into NeedToBeEvaluated.

c) When $b$ is removed from $S$, it is reevaluated only if b $\in$ NeedToBeEvaluated.

These ideas are incorporated into the version of PROPAGATE presented as Algorithm 5.5. 


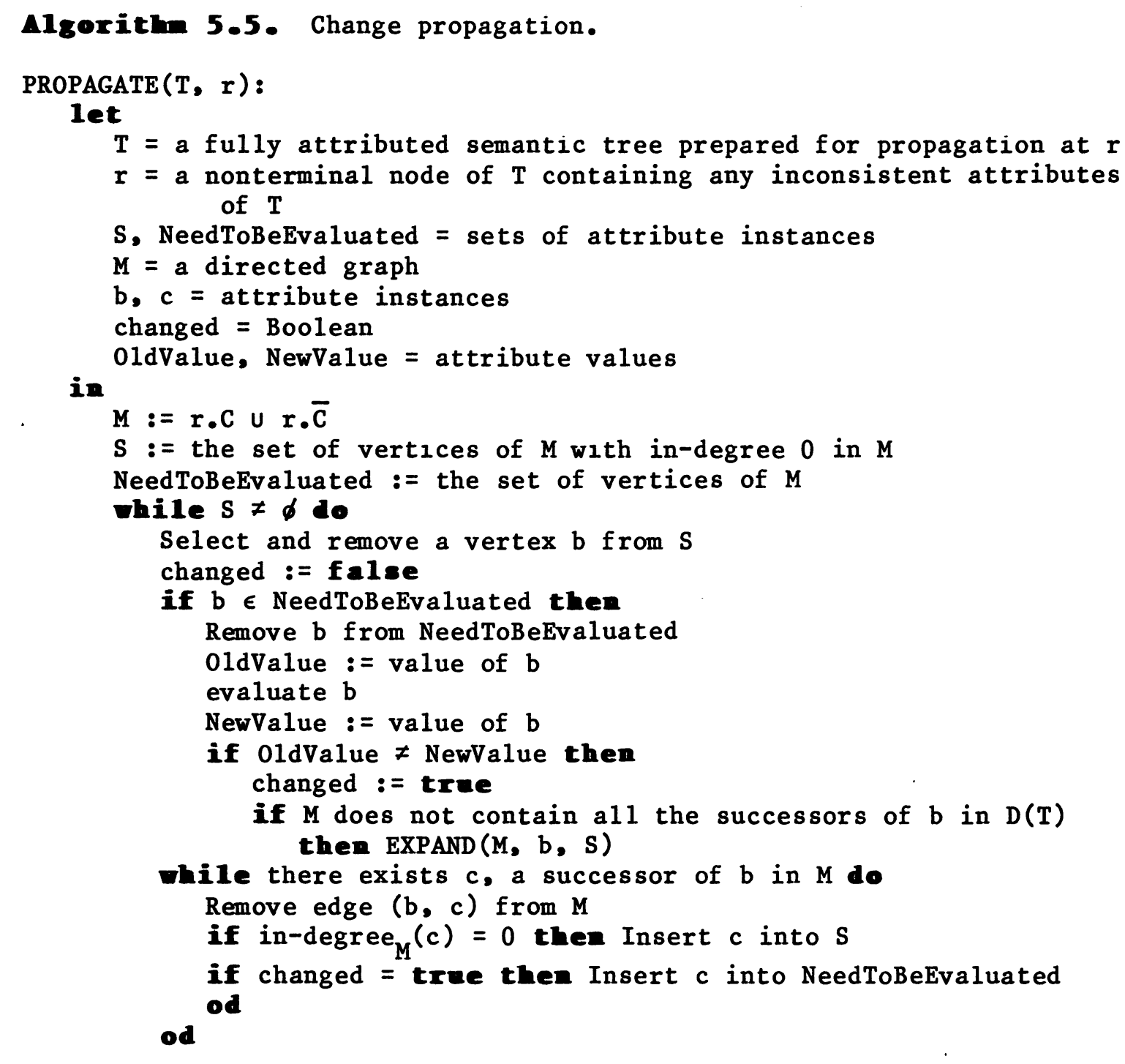

\subsection{Summary}

This chapter has discussed a valuable algorithm if attribute grammars are to be used for building language-based editors. An editor generator using this formalism receives an attribute grammar specification of the language's semantics and context-dependent constraints. Programs are represented internally as consistent, fully attributed semantic 
trees. When a program is altered, consistent values are reestablished throughout the tree in response to each modification.

Each tree has a distinguished location, called the cursor position. The system maintains the invariant that a tree is prepared for propagation at the positıon of its cursor. For a given grammar, moving the cursor to a parent or to a child and reestablishing the invariant has unit cost; a movement of the cursor over a path of length $m$ in the tree costs $O(m)$.

This chapter has presented an algorithm for updating attribute values that is asymptotically optimal in time. This algorithm is used by the basic editing operation, REPLACE, given earlier as Algorithm 4.2, which replaces a subtree of a program tree. 
CHAPTER 6

Extending the model of editing

Nearly all language-based editors represent programs as abstract syntax trees and employ a collection of tree-manipulation primitives, though exactly which operations are included as primitives depends, to a large extent, on the editor's user-interface. So far, the only operations we have discussed are subtree replacement and cursor movement; insertion, deletion, and derivation have been treated as special cases of subtree replacement. Thus, the algorithm presented in the previous chapter is suited for language-based editors with a structural interface like the one used in Emily [Hansen 1971].

This chapter discusses how to extend the ideas presented earlier, so that attribute grammars can be used in more sophisticated languagebased editors.

\subsection{Demand attributes}

Until now, we have abided by the requirement that each attribute in a program tree be given a consistent value after every editing operation. There are some cases, however, where this is not appropriate. For example, consider the case of an editor that provides information about program anomalies. When a program is created, this sort of information is apt to be a nuisance, because one can hardly create a program 
without having introduced temporary anomalies at some point. A more useful facility would be to provide information about anomalies in response to user queries.

We envision providing such facilıties by extending our simple model of editing with a class of demand attributes that are given values only when necessary, that is, when a demand is placed on them for their value. A demand would either arise directly from a user query, from a need to display an attribute on the screen, or from a neighboring attribute needing to use the value as an argument. In some situations, it will be advantageous to intermix demand attributes and regular attributes, so we allow demand attributes to be arguments of regular attributes and vice versa.

Before discussing incremental evaluation of demand attributes, it is worthwhile considering the demand concept as a paradigm for (nonincrementa1) evaluation. Earlier, in chapter 5, we discussed how topological sorting can be turned into an algorithm for attribute evaluation by evaluating a vertex's semantic function when the vertex would normally be given a number in the topological order. However, there is another well-known algorithm for producing a linear ordering of a directed acyclic graph: start from the vertices with no successors, treat the graph as if all edges have been reversed in direction, and do a depth-first search, listing the vertices in endorder. This algorithm can also be turned into an attribute evaluation algorithm by evaluating a vertex's semantic function when the vertex is ready to be given a number in the linear order. This algorithm can be thought of as 
a demand evaluator that fulfills demands for the values of the attributes with no successors.

The demand evaluation algorithm stated below as Algorithm 6.1 uses a set $S$, which is initially the attribute instances of $T$ that have no successors. One by one, demands are placed on members of $S$, which recursively causes demands to be placed on all unevaluated predecessors. When all predecessors finally have values, an attribute is evaluated.

To use demand attributes in language-based editors, we must incorporate into PROPAGATE parts of NULLIFY (of Algorithm 4.4), as well as DemandValue (of Algorithm 6.1). In the version of PROPAGATE stated below as Algorithm 6.2, the demand attributes are treated just 11ke the regular attributes when none of their arguments change value; that is, a

A1gorithn 6.1. Demand evaluation.

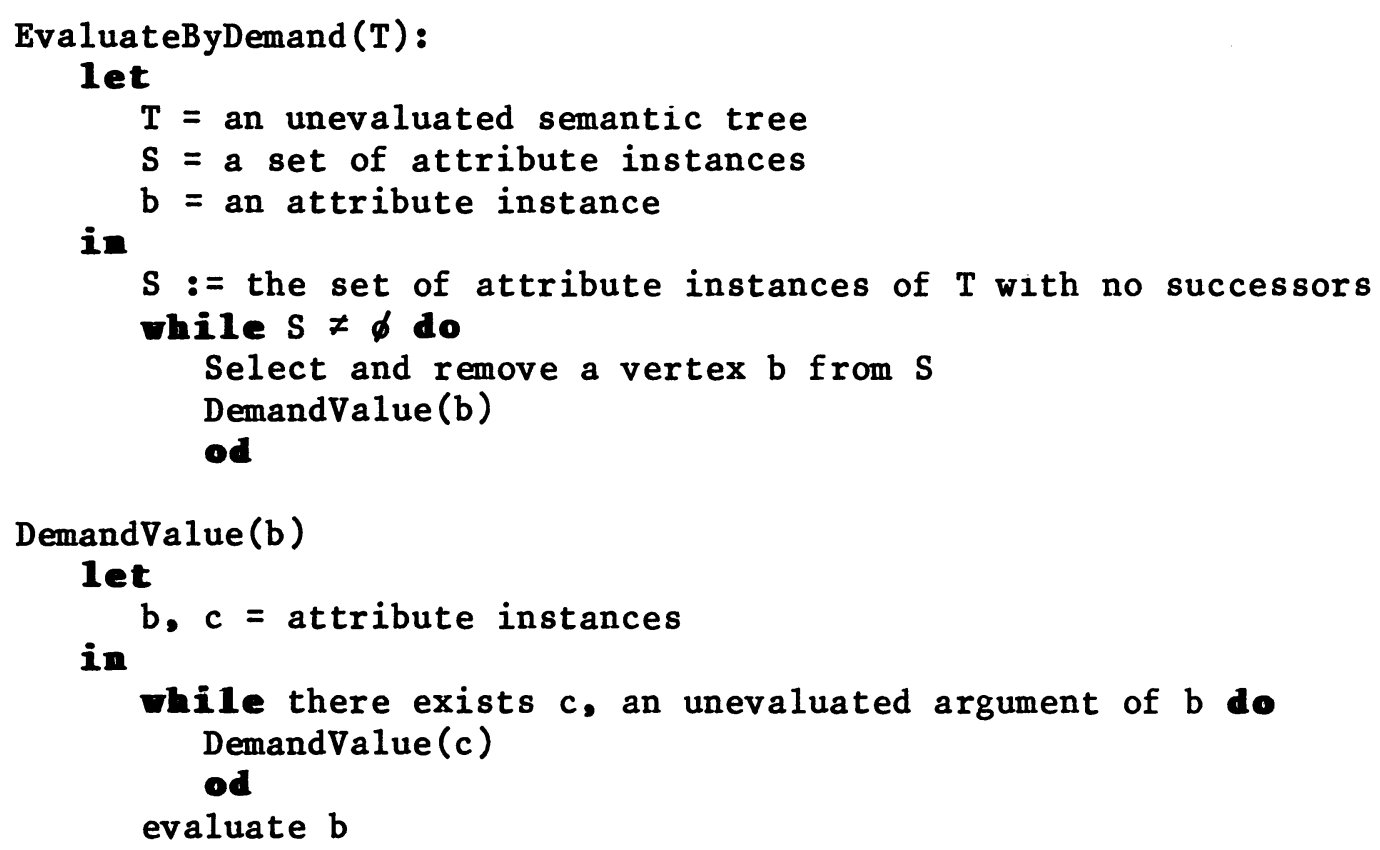


Algorithn 6.2. Change propagation in the presence of demand attributes.

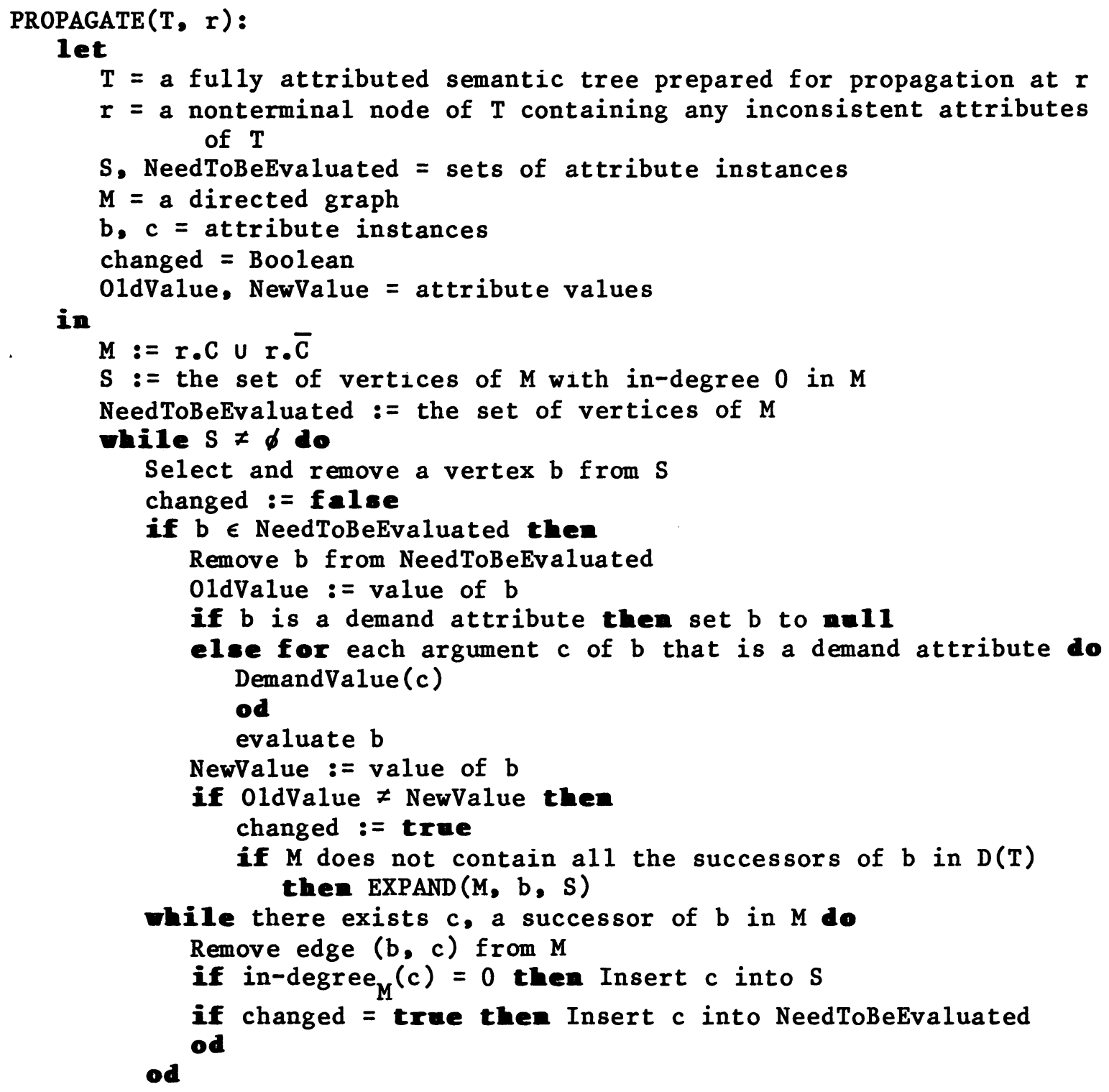

demand attribute keeps its old value if it is not a member of NeedToBeEvaluated when it is removed from the work-11st. If one of its arguments has changed value, a demand attribute is given the value ne11, and receives a value later only if the value is needed for evaluating a regular attribute. 
The subordinate and superior characteristic graphs used by PROPAGATE may be thought of as demand attributes that are linked to the cursor. The computations referred to earlier as side-effects of AscendToParent and DescendToChild are just semantic equations that define two graph-valued attributes:

$$
\mathrm{r} \cdot \underline{\mathrm{C}}:=\text { ExpandedSubordinate }(\mathrm{r}) / \mathrm{A}(\mathrm{r})
$$

$r \cdot \bar{C}:=$ ExpandedSuperior $(r) / A(r)$

When the cursor is moved to node $r$ or when a subtree replacement takes place at node $r, a$ demand is placed on $r . c$ and $r . \bar{c}$.

Our final observation about demand attributes is that user-defined, cursor-linked, demand attributes could be a valuable mechanism for specifying certain facilitıes of language-based editors. For example, error attributes that are incorporated into the program display do not have to be evaluated unless they are actually displayed.

\subsection{Operations other than subtree replacement}

Not all editing operations in language-based editors involve a single subtree replacement. In an editor that supports transformations, operations alter nodes in the interior of a tree, rather than replacing an entire subtree. In an editor that employs an incremental parser, a single program modification may involve a complex restructuring of the program tree through a series of split and join operations [Morris \& Schwartz 1981, Jalıli \& Gallıer 1982].

Although all editing operations in language-based editors can be defined as a sequence of subtree replacements and cursor motions, REPLACE is not suitable for such compositions of operations, because 
REPLACE updates the tree with each subtree replacement. Instead, updating of attribute values should be carried out only after the whole sequence of structural modifications has been completed.

As presented above, PROPAGATE cannot be used to update a tree after an arbitrary modification to a program tree, because until now we have assumed that a11 inconsistencies are confined to the attributes of a single tree-node. This restriction can be relaxed by making a simple change to PROPAGATE. Let $R$ denote the smallest connected region of $T$ that includes all nodes affected by a restructuring; all initially inconsistent attributes of $T$ are attributes of this region. Instead of passing PROPAGATE a single node $r$, we pass $R$; instead of inctializing the model $M$ to $r . C \cup$ r. $\bar{C}$, PROPAGATE initıalizes $M$ to

$$
D(R) \cup \operatorname{root}(R) \cdot \bar{C}\left\{\begin{array}{l}
U \\
\{r \text { on the frontier of } R\}^{r \cdot C}
\end{array}\right.
$$

The set NeedToBeEvaluated is initialized to contain all attributes of $R$; the work-1ist $S$ is initialized to all vertices of $M$ with in-degree 0 . By starting off in this way, $M$ contains all the inconsistent attributes in the tree, and PROPAGATE will be able to update the tree correctly.

Similarly, if the normal-form restriction on attribute grammars is relaxed, then inconsistencies are not restricted to the attributes of $r$ but may occur in attributes of the parent, siblings, and sons of $r$, as we11. By passing PROPAGATE the appropriate $R$, we may drop the normalform restriction that has been assumed in previous chapters.

Note that for a language-based editor that employs an incremental LR parser, we can completely do away with superior characteristic graphs [Horton 1981a]. Each parse restructures the top portion of a program 
tree, all the way to the root of the tree. Because $R$ includes the root, M can never expand upward; thus, superior characteristic graphs are never needed. 
CHAPTER 7

Space Efficient Attribute Evaluation

The chief hindrance to the widespread adoption of attributegrammar-based systems has been their profligate consumption of storage. Previous work on attribute evaluation has been mostly devoted to designing time-efficient evaluators, and much less has been done concerning space-efficient evaluators. This chapter and the two chapters that follow present new techniques for storage management.

Methods that have previously been suggested for making more efficient use of storage fall into several categories, and not all techniques are compatible with one another. The objective of some methods is to reduce the cost of storing the derivation tree. Schulz proposes linearizing the derivation tree so that the evaluator can make use of secondary storage for storing the tree [Schulz 1976]. Jazayeri and Pozef sky propose a method for constructing files of attribute-evaluation instructions during parsing as a means of completely avoiding the need to build and store the derivation tree [Jazayeri \& Pozefsky 1977]. Both of these techniques can be applied only when evaluation is carried out by an alternating-pass evaluator.

Other techniques are possible when the purpose of attribute evaluation is to obtain the value of a distinguished attribute of the derivation tree, such as one of the synthesized attributes of the root. In 
this situation, there are ways of reducing the amount of space devoted to storing attribute values because storage does not have to be allocated for an attribute until it is defined, and storage can be reclaimed when its value has been used for the last time [Lorho 1977]. To reduce the amount of space needed for storing attribute values even further, Räihä proposes a method in which all attribute values in a chain of attribute instances defined by identity functions share the same storage area; after all members of one chain are no longer needed, the storage .can be used for another chain [Räihä 1979].

These techniques are dynamic in the sense that decisions about when to allocate and reclaim storage depend not only on the grammar, but also on the particular tree that is being evaluated. Alternative techniques are static in the sense that such decisions are made at constructiontime by statically analyzing the grammar. Whereas dynamic techniques can make decisions about how storage is used for individual attribute instances, static techniques must deal with classes of attributes, where usually each class consists of all of the instances of a particular attribute. In alternating-pass evaluators, for example, Jazayeri and Pozefsky propose allocating space for all instances of an attribute on the first pass during which any of the instances is defined, and reclaiming the space on the last pass during which one of the attribute instances is used [Jazayeri \& Pozefsky 1982].

This technique allocates different storage areas for different members of a class; a different approach is to use a single storage area for all members of the class. Ganzinger investigated the feasibility of implementing more than one attribute class with a single storage area. 
His results indicate that an automatic technique for making optimal assignments of attribute classes to storage areas would not be practical; he showed that automatically determining an allocation of attribute classes to storage areas that minimizes the number of storage areas is NP-complete [Ganzinger 1979].

This chapter presents two new dynamic algorithms for evaluating a distinguished attribute of a derivation tree. These algorithms make no attempt to achieve any sort of optimal behavior, and, in contrast to most previous techniques, these algorithms may evaluate an attribute more than once; however, the characteristic that sets both algorithms apart from previous evaluation methods is that they have sublinear behavior, even in the worst case. Section 7.2 describes an algorithm that saves at most $O(\sqrt{n})$ attribute values at any stage of evaluation, where $n$ is the number of attributes in the derivation tree; Section 7.3 describes an algorithm that saves at most $0(\log n)$ attribute values at any stage of evaluation.

Note that because these techniques only apply when the purpose of attribute evaluation is to obtain the value of a single distinguished attribute, they are not compatible with the model of language-based editing that was introduced in Chapter 4. Chapters 8 and 9 are concerned with two other aspects of efficient storage management, and the techniques presented in those chapters are compatible with that model.

\subsection{Motivation}

As originally employed by Knuth, an attribute grammar specifies the translation of a context-free language into a semantic domain. The 
attribute grammar implicitly defines how each attribute is assigned a value; the meaning of a string is defined to be the value of a distinguished synthesized attribute of the root of the string's semantic tree. In this situation, the evaluator must produce the value of the meaning attribute, but need not retain the value of any of the other attributes.

The process of evaluating a semantic tree can be modeled as a pebbling game on the tree's dependency graph, where a pebble corresponds to a stored attribute value. The game has two rules:

a) A vertex can be pebbled whenever there are pebbles on all of its predecessors.

b) A pebble can be removed at any time.

The objective of the pebbling game is to pebble the vertex that corresponds to the meaning-attribute, while obeying the constraint that at any one time, at most a given number of vertices are pebbled.

The pebbling game has been used for analyzing a wide range of computational situations because it provides a convenient way of studying a problem's space and time requirements. ${ }^{1}$ space is studied in terms of the maximum number of vertices pebbled simultaneously during the game; time is studied in terms of the number of steps taken during the game.

It has previously been established that an arbitrary directed acyclic graph can be pebbled with $0(n / \log n)$ pebbles [Hopcroft et al. 1977], and that there exist graphs that require

\footnotetext{
$1_{\text {[Pippenger 1980] is an excellent survey of the literature on }}$ pebbling.
} 
$\varrho(n / \log n)$ pebbles $^{2}$ [Paul et a1. 1977]. It has also been established that planar graphs can be pebbled with $O(\sqrt{n})$ pebbles [Lipton \& Tarjan 1977], and that there exist graphs that require $\varrho(\sqrt{n})$ pebbles [Cook 1974]. These lower-bound results, however, are not applicable to attribute evaluation because attribute grammars allow only limited kinds of dependencies between attributes; neither the graphs that establish the $\Omega(\sqrt{n})$ lower bound for planar graphs, nor the graphs that establish the $\varrho(n / \log n)$ lower bound for arbitrary graphs are possible attribute dependency graphs.

Attribute dependency graphs are not, in general, planar; consequently, they cannot, in general, be pebbled by the $O(\sqrt{n})$ method for pebbling planar graphs. The dependency graph $D(T)$ of a tree $T$ is composed of the dependency graphs of the production instances of $T$. Clearly, $D(T)$ cannot be planar if one of these subgraphs is non-planar, and it is a simple matter to construct a production with a non-planar dependency graph. For example, the dependency graph of the following production is the non-planar graph $\mathrm{K}_{3,3}$ [Harary 1969, p.104]:

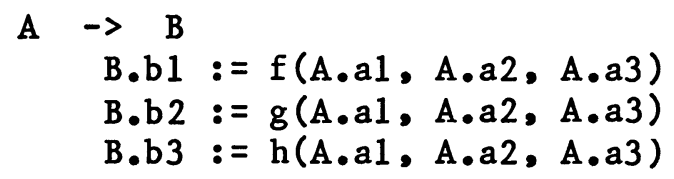

a1 a2 a3

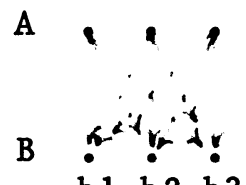

b1 b2 b3

The attribute evaluation algorithms described in earlier chapters pebble the vertices of a dependency graph in topological order without

\footnotetext{
The notation $g(n)=\Omega(f(n))$, which means $\lim \sup [g(n) / f(n)]>0$, signifies that $g(n)$ is asymptotically of an order at least as great as $f(n)$.
} 
removing any pebbles; an n-vertex dependency graph is pebbled in $n$ steps using $n$ pebbles. This chapter discusses pebbling a vertex of a dependency graph using $o(n)$ pebbles by possibly using more than $n$ steps. ${ }^{3}$ The algorithm presented in Section 7.2 uses $O(\sqrt{\mathrm{n}})$ pebbles and no more than $O(n)$ steps. The algorithm presented in Section 7.3 uses only $O(\log n)$ pebbles but uses a quadratic number of steps.

It is important to understand that, by itself, an algorithm that evaluates a semantic tree using $o(n)$ pebbles does not necessarily use $o(n)$ space. Pebbling gives a precise measure of the space and time costs of attribute evaluation only when the following two conditions are met :

a) Each semantic function application requires a constant amount of time and space.

b) The space required to store each attribute value is bounded by a constant.

Despite the fact that neither of these conditions is necessarily true in practice, the pebbling game is still a useful heuristic method for studying attribute evaluation; even when the space required to store each attribute value is not bounded by a constant, an o(n) pebbling method conserves storage by reducing the number of values that need to be retained during evaluation.

A further problem with using the pebbling game to analyze the way storage is utilized during evaluation is that pebbles account only for

\footnotetext{
3 The notation $g(n)=o(f(n))$, which means $\lim \sup [g(n) / f(n)]=0$, signifies that $g(n)$ is asymptotically of lower order than $f(n)$.
} 
the space used to store attribute values, and fail to account for all other space used, such as that used for deciding the order in which attributes are to be evaluated. In the discussion below, we will be careful to show that the amount of additional space used is of the same order as the number of pebbles used, under the following assumptions:

a) We assume that we have been furnished a semantic tree to evaluate, and in analyzing the amount of storage used during evaluation we will not count the storage used to represent the tree.

b) We assume that each nonterminal in the attribute grammar has at least one attribute; thus, the relationship between $n$, the number of attribute instances in the semantic tree, and $m$, the number of nodes in the semantic tree, is given by:

$$
\mathrm{m} \leq \mathrm{n} \leq(\mathrm{m}) \text { (MaxAttrs) }
$$

where MaxAttrs is the maximum number of attributes of any nonterminal of the grammar.

c) We assume that a tree node contains references to each of its children, that each node is labeled with a descriptor that can be used to determine the node's arity, and that a small number of bits are associated with each node so that insertion, deletion, and membership operations on a set of tree nodes can be implemented as unittime operations.

In no case is the total amount of uncounted space more than $O(n)$. 
7.2. An evaluation algorithm that saves at most $Q(\sqrt{n})$ values

This section describes a space-efficient method for evaluating a distinguished attribute of an n-attribute tree. At any one time, this method saves at most $O(\sqrt{n})$ attribute values, and uses at most $O(\sqrt{n})$ units of additional space; altogether, the method performs at most $O(n)$ function applications. For each of these terms, the constant of proportionality depends only on the quantities MaxAttrs and MaxSons (the maximum number of nonterminals on the right-side of any production in the attribute grammar), both of which are constants for a given grammar.

The evaluation method is a divide-and-conquer algorithm that makes use of the concept of a separator set: a set of vertices $A$, is a separator set of a graph $G$ if we can partition the vertices of $G$ into $A$ and two other sets, $B$ and $C$, such that no edge connects a vertex of $B$ to a vertex of $C$, or vice versa. We say that $A$ is a $k$-separator set if we can partition the vertices of $G$ into $A$ and $k$ other $\operatorname{sets} B_{1} \cdot B_{k}$, such that no edge connects a vertex of $B_{i}$ to a vertex of $B_{j}$, for $i \neq j$.

The $\sqrt{n}$-evaluation algorithm relies on the fact that attribute dependency graphs contain small separator sets. In general, this is a property that neither arbitrary dags nor planar dags possess, but because each attribute instance in a semantic tree depends only on attribute instances in a single production instance, the only attributes in the subtree rooted at node $X$ that can depend on attributes outside the subtree at $X$ belong to $A(X)$; similarly, the only attributes outside the subtree rooted at $X$ that can depend on attributes in the subtree rooted at $X$ belong to $A(X)$. Thus $A(X)$, the set of attribute instances associated with node $x$, is a separator set of the tree's dependency 
graph. The attributes of each tree node is a separator set of the dependency graph, and each of these separator sets is bounded in size by MaxAttrs.

The existence of these separator sets allows attribute evaluation to be carried out using a divide-and-conquer strategy. The task of evaluating a distinguished attribute $b$ is divided into smaller tasks by first finding a separator set of the dependency graph, and then letting each separator-set element be the distınguished attribute of a component of the dependency graph. On the completion of each of these subproblems, the value of the distinguished attribute is saved, but the storage for all other attribute values in the component is reclaimed.

To ensure that the work involved in solving each subproblem is confined to a single component of the dependency graph, we employ the same trick used in the Lipton-Tarjan method for pebbling a planar graph: the subproblems are treated in an order that respects the order of separator set elements in the partial order given by $D(T)$. When it comes time to solve the subproblem with distinguished attribute $c$ of the separator set, every ancestor of $c$ that belongs to the separator set has already been evaluated. It will be shown that the arguments of $c$ either belong to the separator set, or else they are contained in exactly one component of $D(T)$, cut of from the rest of $D(T)$ by separator-set elements; consequently, each subproblem is no larger than a single component of $\mathrm{D}(\mathrm{T})$

The partitioning step of the $\sqrt{n}$-evaluation method produces a group of $O(\sqrt{n})$ subproblems, each of size $O(\sqrt{n})$. A problem of size $O(\sqrt{n})$ can be solved using at most $O(\sqrt{n})$ attribute values by evaluating attributes 
in a topological order of the dependency graph, without discarding any values. Once a subproblem is solved, we can release the space used to store all values of the subproblem, except for the value of the distinguished attribute. Because there are at most $O(\sqrt{\mathrm{n}})$ subproblems, there are at most $O(\sqrt{n})$ separator-set values to be saved; thus, at most $O(\sqrt{n})$ values are needed altogether.

Note that unlike the Lipton-Tarjan method for pebbling a planar graph, the $\sqrt{n}$-evaluation algorithm is not recursive; each of the subproblems is solved in a straightforward manner without partitioning the problem still further. Recursion is used in the algorithm described in Section 7.3 , which saves at most $0(\log n)$ attribute values at any one time; however, the $\sqrt{\mathrm{n}}$-evaluation algorithm takes no more than a linear number of steps, whereas the recursive, $O(\log n)$-evaluation algorithm uses a quadratic number of steps.

The $\sqrt{n}$-evaluation algorithm has three parts: a partitioning step, a projection step, and an evaluation step. The partitioning step, described in Section 7.2.1, finds a suitable collection of subproblems to solve. The projection step, described in Section 7.2.2, generates an order in which to solve the subproblems that ensures that each subproblem is smaller than the original problem. The evaluation step, described in Section 7.2 .3 , then simply solves the subproblems in the required order.

\subsubsection{Partitioning}

Rather than partitioning the problem according to the structure of the dependency graph, we partition the problem according to the 
structure of the derivation tree. A set of nodes $P$ partitions a tree $T$ into components, where a component is a maximal-size, connected region of $T$ in which none of the interior nodes are elements of $P$. For example, the set $\{E, C\}$ partitions the tree:

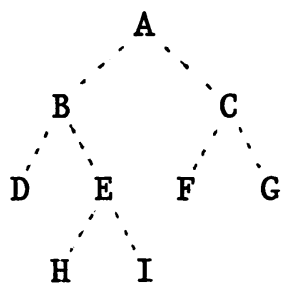

into the three components:

(1)<smiles>[B]PB([B])[CH]C</smiles>

(2)

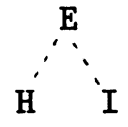

(3)

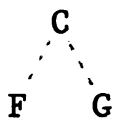

The goal of the partitioning step is to divide the derivation tree into $O(\sqrt{n})$ components that are each no larger than $O(\sqrt{n})$ nodes and that each adjoin at most MaxSons +1 other components. This is accomplished in three phases: the first phase partitions the derivation tree into components of maximum depth $\lfloor\sqrt{\mathrm{m}}\rfloor$, where $m$ is the number of nodes in the tree; the second phase repartitions the tree into components of size $O(\sqrt{\mathrm{m}})$; the third phase further subdivides the components found during the second phase so that each component adjoins at most MaxSons +1 other components. 
Although each phase subdivides the tree according to $\mathrm{m}$, each node can have no more than MaxAttrs attributes, so $m$ is related to $n$ by:

$$
\mathrm{m} \leq \mathrm{n} \leq(\mathrm{m}) \text { (MaxAttrs) }
$$

Consequent 1y,

$$
\sqrt{\mathrm{m}} \leq \sqrt{\mathrm{n}} \leq(\sqrt{\mathrm{m}})(\sqrt{\operatorname{MaxAttrs}})
$$

and $O(\sqrt{\mathrm{m}})=O(\sqrt{\mathrm{n}})$.

Phase 1.

The first phase of partitioning is carried out by the procedure PartitionByDepth, stated as Algorithm 7.1. PartitionByDepth performs a scan of the tree, starting from the root, partitioning the tree into components whose maximum depth is $\lfloor\sqrt{\mathrm{m}}\rfloor$ by finding all nodes whose depth is a multiple of $\lfloor\sqrt{\mathrm{m}}\rfloor$.

To bound the storage overhead of the scan, we make use of a method developed by Lindstrom for traversing a tree using only two additional storage locations [Lindstrom 1973]. These two locations, named CurrentNode and PreviousNode, point to the node currently being visited and the node visited on the previous step, respectively. During scanning, each node $X$ is visited $N_{X}+1$ times, where $N_{X}$ is the number of children belonging to $x$. On each visit, a cyclic permutation of PreviousNode, CurrentNode, and the fields reserved at $X$ for storing child pointers is performed, advancing CurrentNode to the next node in the scan.

The first visit to $X$ initiates the scan of the subtree rooted at $X$; on this visit, the address of the parent of $\mathrm{X}$ is stored in PreviousNode and the addresses of $X^{\prime} s$ children are stored in the child-pointer fields 
A1gorithn 7.1. Partition $T$ into components whose maximum depth is $\lfloor\sqrt{m}\rfloor$, where $m$ is the number of nodes in $T$.

PartitionByDepth $(T)$ :

let

$T=a$ derivation tree

$\mathrm{m}$, Depth = integers

Partition, PathToRoot $=$ sets of tree nodes

in

CurrentNode, PreviousNode $=$ tree nodes

Partition : $=\varnothing$

$\mathrm{m}:=$ the number of nodes in $\mathrm{T}$

PreviousNode : = nil

CurrentNode $:=\operatorname{root}(\mathrm{T})$

PathToRoot $:=\varnothing$

Depth : $=0$

-hile CurrentNode $\neq$ nil do

Insert CurrentNode into PathToRoot

Cyclically permute PreviousNode, CurrentNode, and the child pointers at CurrentNode

if CurrentNode $\epsilon$ PathToRoot or CurrentNode $=$ nil then

Depth := Depth - 1

Remove PreviousNode from PathToRoot

if Depth +1 is a multiple of $\lfloor\sqrt{\mathrm{m}}\rfloor$ then

Insert PreviousNode into Partition

else Depth : $=$ Depth +1

od

return(Partition)

of $X$. Each subsequent visit to $X$ occurs after a subtree rooted at one of X's children has been completely scanned; on each of these visits, the address of the parent of $X$ and the addresses of $X^{\prime} s$ children are again stored in PreviousNode and the child-pointer fields of $\mathrm{X}$, but in a different cyclic permutation each time. The scan of the subtree rooted at $X$ terminates after the $\left(N_{X}+1\right)^{\text {st }}$ visit to $X$ because $N_{X}+1$ permutations restores all the child pointers to their original locations, and sets CurrentNode to the parent of $X$. 
During Lindstrom scanning, the $\mathrm{N}_{X}+1$ visits made to a node cannot be distinguished from one another without some sort of extra information. Algorithm 7.1 makes use of the set PathToRoot in order to treat nodes of the tree in endorder. At each step, PathToRoot consists of the nodes along the path from CurrentNode to the root of the tree. After each cyclic permutation of pointer information, we can determine whether CurrentNode has advanced to a new node or whether it has retreated to a previously visited node by testing whether it has landed at a node that belongs to PathToRoot. If it has, then the node visited on the previous step was just visited for the last time; taking the appropriate action at PreviousNode whenever this condition occurs allows nodes to be treated in endorder. ${ }^{4}$ Because the only use made of PathToRoot is to test nodes for membership, PathToRoot can be implemented using a single bit at each tree node and no other additional storage.

In Algorithm 7.1, the set Partition consists of all the nodes that precede CurrentNode in endorder that are at a depth that is a multiple of $\lfloor\sqrt{\mathrm{m}}\rfloor$. To determine if this condition is true for a node, Algorithm 7.1 keeps track of the depth of CurrentNode in the variable Depth. PartitionByDepth builds the set Partition by considering nodes in endorder, inserting PreviousNode into Partition if Depth +1 is a multiple of $\lfloor\sqrt{\mathrm{m}}\rfloor$. PartıtionByDepth makes a single pass over the tree, and does a constant amount of work at each node, so its total running time is $0(n)$.

${ }^{4}$ For brevity, similar traversals in algorithms given later in this chapter are specified using the notation:

for each node $X$ in an endorder traversal of $T$ <action> 
Although the number of elements in Partition can be far more than $O(\sqrt{\mathrm{m}})$, the only use that is made of Partition is to test nodes for membership in it, so Partition can be implemented using a single bit at each tree node and no other additional storage.

Phase 2.

The second phase of partitioning uses the components found during the first phase to repartition the tree into components of size $0(\sqrt{\mathrm{m}})$. Because each component defined by the depth-partition is no deeper than $\lfloor\sqrt{\mathrm{m}}\rfloor$, an individual component can be traversed by a recursive procedure that involves no more than $\lfloor\sqrt{\mathrm{m}}\rfloor$ levels of recursion, and thus no more than $O(\sqrt{\mathrm{m}})$ units of stack space.

The procedure RepartitionBySize, given as Algorithm 7.2, constructs the set NewPartition bottom-up, by considering the components defined by 0ldPartition in endorder, traversing each component in endorder with the recursive routine RepartitionComponent. NewPartition is initially empty, and RepartitionComponent adds a new node to NewPartition whenever a node is found that is at the root of a component larger than $\sqrt{\mathrm{m}} /$ MaxSons.

For each node $Y$, RepartitionComponent computes how large a component there would be if $Y$ were the root of a component. Each recursive call on RepartitionComponent $\left(Y_{i}\right)$, where $Y_{i}$ is the $i^{\text {th }}$ child of $Y$, either returns 1 , if $\mathrm{Y}_{i}$ belongs to NewPartition, or it returns how large a component there would be if $\mathrm{Y}_{i}$ were the root of a component; thus, the quantity returned from each call ranges from 1 to $\sqrt{\mathrm{m}} /$ MaxSons. The size of the component rooted at $\mathrm{Y}$ is computed by adding 1 (for $\mathrm{Y}$ itself) to 
Algorithn 7.2. Given 01dPartition, a set of nodes in $T$, build NewPartition, a set of nodes that partitions $T$ into components of size $O(\sqrt{m})$, where $m$ is the number of nodes in $T$.

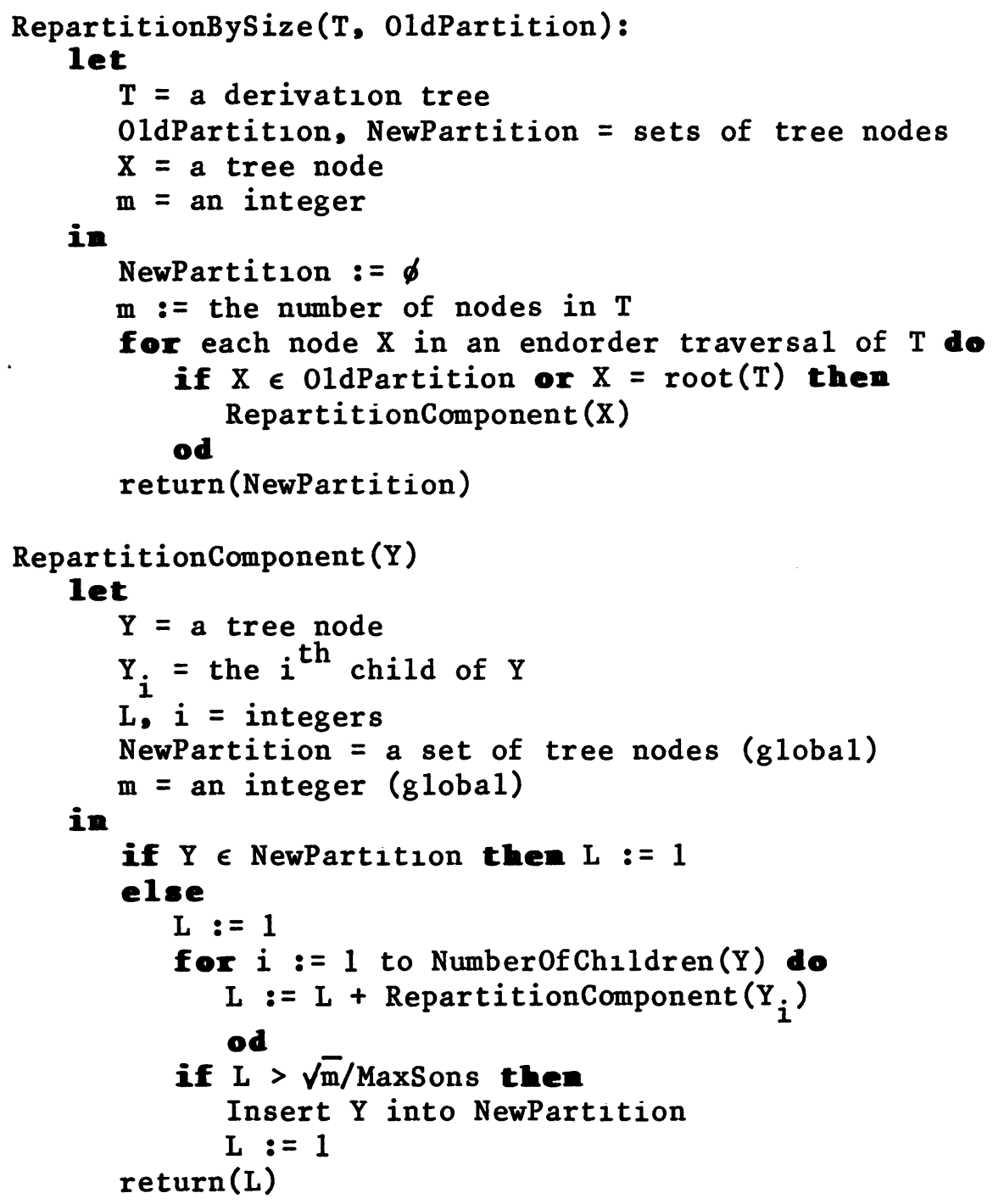

the sum of the values returned by the calls to RepartitionComponent with $Y^{\prime}$ s children. Because $Y$ has at most MaxSons children, the number of nodes in the component can be no larger than $\sqrt{\mathrm{m}}+1$.

RepartitionComponent only stops descending when it encounters a member of NewPartition, so each of the calls on RepartitionComponent 
made by RepartitıonBySize is not confined to just a single one of the components defined by 0ldPartition. However, when 0ldPartition is the $\lfloor\sqrt{\mathrm{m}}\rfloor$-depth partition found during the first phase of partitionıng, each node $\mathrm{Y}$ is visited at most twice. $\mathrm{Y}$ is visited the first time during a call initiated on the nearest ancestor of $Y$ that belongs to 0ldPartition; $Y$ may be visited a second time during a call initiated on the next-to-nearest ancestor of $Y$ that belongs to 0ldPartition. Because no node is visited a third time, the total running time of Algorithm 7.2 is $.0(\mathrm{~m})$.

Except for the component containing the root of the tree, the root of each component is shared by exactly one other component; thus, for the set of components $C_{P}$, defined by a partition $P$, the sum of the components' sizes is related to the total number of nodes $m$ by:

$$
\mathrm{m}=1+\sum_{c \in \mathrm{C}_{\mathrm{P}}}(|c|-1)
$$

Except possibly for the component containing the root of the tree, each component defined by the set NewPartition of Algorithm 7.2 contains at least $\sqrt{\mathrm{m}} /$ MaxSons +1 nodes, so

$$
m \geq 1+\left(\left|C_{\text {NewPartition }}\right|-1\right)(\sqrt{\mathrm{m}} / \text { MaxSons })
$$

Solving for $\left|C_{\text {NewPartition }}\right|$, we get:

$$
\begin{aligned}
\left|\mathrm{C}_{\text {NewPartition }}\right| & \leq \frac{(\mathrm{m}-1) \text { MaxSons }}{\sqrt{\mathrm{m}}}+1 \\
& <(\text { MaxSons })(\sqrt{\mathrm{m}})+1
\end{aligned}
$$

Thus, the second phase of partitioning finds no more than (MaxSons) $(\sqrt{\mathrm{m}})+1$ components, each containing no more than $\sqrt{\mathrm{m}}+1$ nodes. 
Phase 3.

The components found during the second phase of partitioning may be adjacent to as many as $O(\sqrt{\mathrm{m}})$ other components. An additional partitioning phase is needed because this condition would cause the projection step described in the next section to use too much storage. The third phase of partitioning further subdivides the components from the second phase of partitioning by adding to the partition set every node that is a lowest common ancestor of some pair of partition nodes. This process makes each component adjacent to no more than MaxSons +1 other components, and at most doubles the size of the old partition set.

The procedure AddAncestors, stated as Algorithm 7.3, constructs the set NewPartition bottom-up by considering the components defined by 01dPartition in endorder, traversing each component with the recursive routine AddAncestorsInComponent. NewPartition is initially empty, and AddAncestorsInComponent adds a new node to NewPartition whenever a node is found that is the lowest common ancestor of some pair of nodes that belong to 0ldPartition. Note that because for each node $X$ LowestCommonAncestor $(X, X)=X$, every node in 0ldPartition is put in NewPartition.

For each node $Y$, AddAncestors InComponent checks if $\mathrm{Y}$ is the lowest common ancestor of some pair of nodes that belong to 01dPartition. Each recursive call on AddAncestorsInComponent $\left(x_{i}\right)$, returns trae if the subtree rooted at $x_{i}$ contains a member of 0ldPartition. If this is true of more than one child of $X$, then $X$ is the lowest common ancestor of some pair of nodes in 0ldPartition, and is added to NewPartition. 


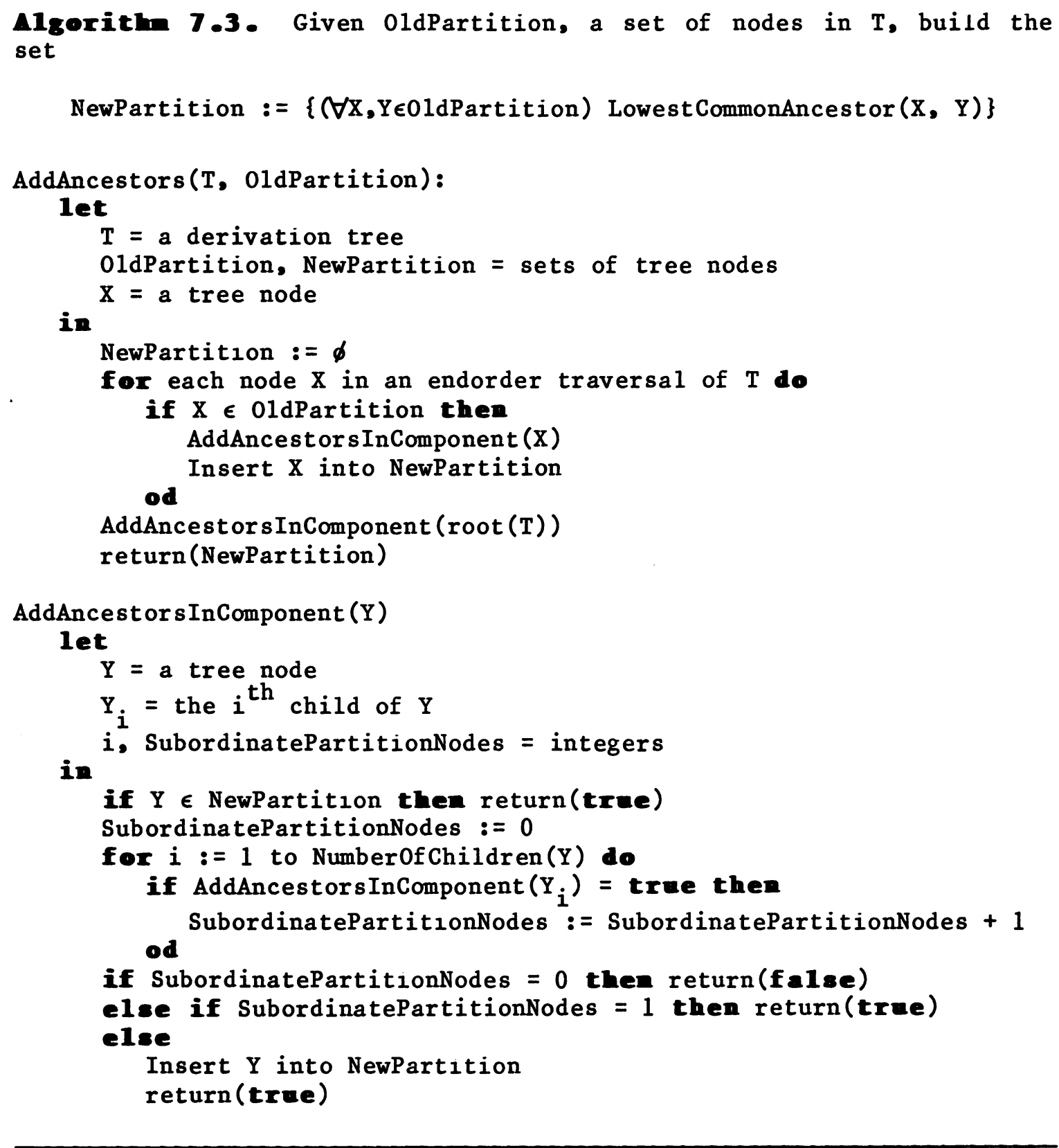

The partition set computed by this process has two important properties:

a) The number of elements in NewPartition is at most double the number of elements in 0ldPartition. 
b) Each component defined by NewPartition is adjacent to at most MaxSons +1 other components.

When 0ldPartition is the partition set from the second phase of partitioning, the components defined by 01dPartition are no larger than $O(\sqrt{\mathrm{m}})$, and AddAncestorsInComponent traverses a component recursively using no more than $O(\sqrt{\mathrm{m}})$ units of additional storage.

Because each member of 01dPartition is made a member of NewPart1tion, each of the calls on AddAncestorsInComponent made by AddAncestors is confined to a single one of the components defined by 0ldPartition. Each member of 01dPartition causes one call on AddAncestorsInComponent; thus, the total running time of Algorithm 7.3 is $0(m)$.

\subsubsection{Projection}

The $\sqrt{n}$-evaluation algorithm uses a divide-and-conquer strategy to evaluate a distinguished attribute of a derivation tree. This problem is divided into subproblems using the methods described in the previous section to partition the tree. Each subproblem involves evaluating an attribute of one of the partition set nodes, treating it as the distinguished attribute of a component of the tree.

To ensure that solving each subproblem involves only evaluations of attributes that are part of a single component, the order in which subproblems are solved must respect the order of the distinguished attributes of the subproblems in the partial order given by the dependency graph $D(T)$. By treating the subproblems in this order, when it comes time to solve the subproblem with distinguished attribute $b$, every ancestor of $b$ that is an attribute of the partition set $P$ already has a 
value. Because an attribute is defined solely in terms of attributes of a single production, the arguments of $b$ are either members of $A(P)$, or they are contained in exactly one component of $D(T)$, cut off from the rest of $D(T)$ by members of $A(P)$; consequently, the work involved in solving each subproblem is confined to a single component.

This order is found by constructing the graph $D(T) / A(P)$ with the procedure Project, which is stated below as Algorithm 7.4. Project constructs the graph $D(T) / A(P)$ by taking each of the components defined by $P$, projecting the dependency graph of the component onto the attributes of the partition nodes in the component, and merging the projected graphs together. When the number of nodes in $P$ is no more than $0(\sqrt{\mathrm{m}})$, the size of the $\operatorname{graph} \mathrm{D}(\mathrm{T}) / \mathrm{A}(\mathrm{P})$ is no more than $O(\sqrt{\mathrm{m}})$; thus, the total space cost of Algorithm 7.4 is $0(\sqrt{\mathrm{m}})$.

Algorithn 7.4. Build the graph $G:=D(T) / A(P)$.

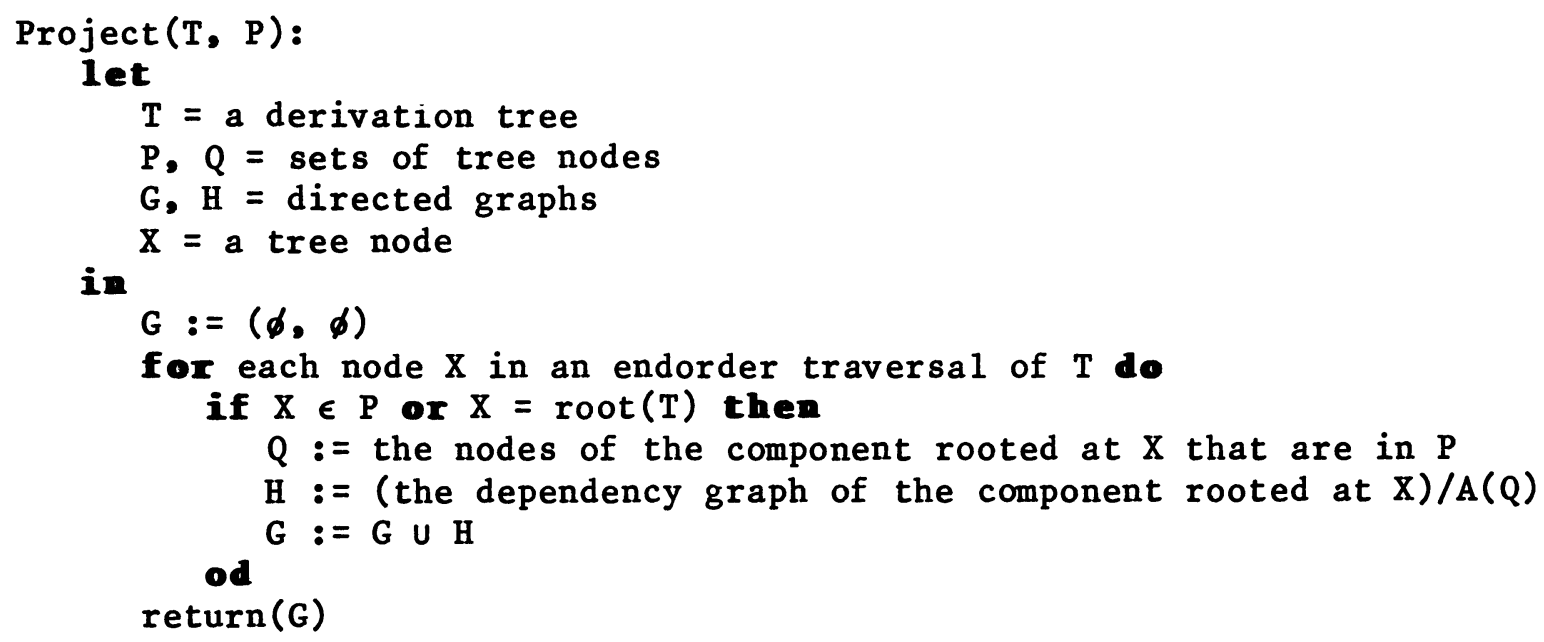


The projection operation for each component can be done by a depth-first search from each inherited attribute of the root of the component and each synthesized attribute of partition nodes at leaves of the component. When each component is no larger than $0(\sqrt{\mathrm{m}})$, and when there are no more than MaxSons +1 partition nodes that are part of each component, the running time of this operation is no more than $0(\sqrt{\mathrm{m}})$; given $O(\sqrt{\mathrm{m}})$ components, the total running time of Algorithm 7.4 is $0(\mathrm{~m})$.

\subsubsection{The attribute evaluation algorithm}

The procedures described in the previous two sections are used by the procedure Evaluate, stated as Algorithm 7.5, to divide an evaluation problem of size $n$ into a group of $0(\sqrt{n})$ subproblems of maximum size $O(\sqrt{n})$. Algorithms $7.1,7.2$, and 7.3 are used to find a set of nodes $P$ whose attributes $A(P)$ are an $O(\sqrt{n})$-separator set of the dependency graph

Algorithn 7.5. Given a semantic tree $T$ and a distinguished attribute $b$, evaluate $b$.

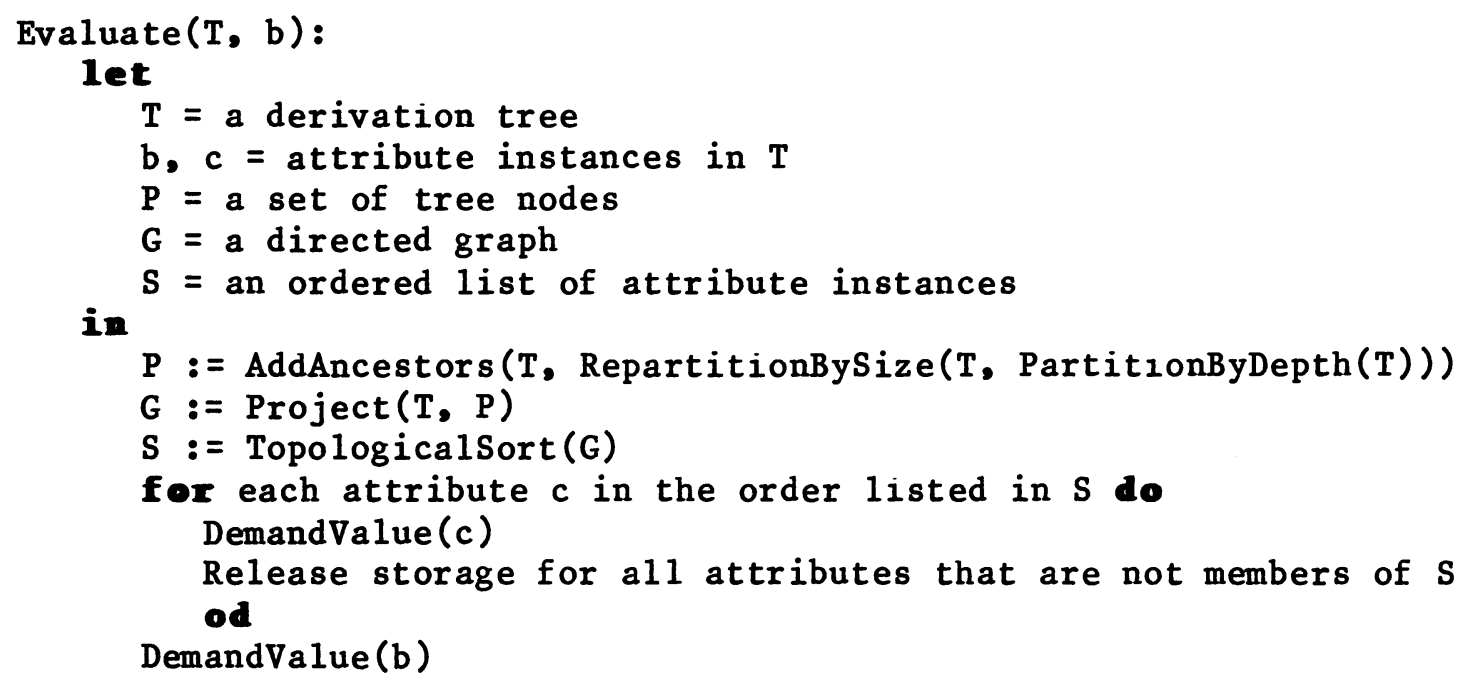


$D(T)$. Algorithm 7.4 is used to construct the graph $G:=D(T) / A(P)$, and by topologically sorting $G$, we find an order for the members of $A(P)$ that respects their order in $D(T)$, which is used to schedule calls on DemandValue, the recursive procedure for evaluating an attribute by demand that was used in Algorithm 6.1.

Because the evaluation order of the separator-set elements respects their order in $D(T)$, when attribute $c$ is evaluated, every ancestor of $c$ that is a separator set element has been given a value. This restricts each semantic function application made during a call on DemandValue to a single component of $D(T)$, so at most $O(\sqrt{n})$ steps are used to evaluate at most $O(\sqrt{n})$ attributes. Only the values of separator set elements are retained after each call on DemandValue; because Evaluate makes $O(\sqrt{n})$ calls on DemandValue, the total number of values retained at any time during evaluation is bounded by $O(\sqrt{n})$, and the total number of steps used is bounded by $O(n)$.

\subsubsection{Discussion}

Algorithm 7.5 is potentially a practical evaluation method because it provides a way of making efficient use of both primary and secondary storage. If each of the components defined by the partition $P$ in Algorithm 7.5 is linearized and stored in secondary storage, then the remainder of the algorithm never needs to have more than one component in primary storage at any time, so no more than $0(\sqrt{n})$ units of primary storage are needed to carry out evaluation.

One problem that needs to be considered further is how to separate the tree into the components that are stored in contiguous secondary 
storage without overflowing primary storage. If the parser were to place a linearized representation of the derivation tree in secondary storage, then we need a way of efficiently splitting this into components. An alternative would be to find a way of splitting the tree into components as part of parsing, which would make it possible to write individual components to secondary storage directly.

It is interesting to compare the $\sqrt{n}$-evaluation method with the method proposed by Schulz for using secondary storage in an alternating-pass evaluator [Schulz 1976]. Schulz's method uses a linearized representation of the program tree in an intermediate file in secondary storage. On every even-numbered pass, the tree is stored in left-to-right preorder, and on every odd-numbered pass, it is stored in right-to-left preorder. The evaluator performs alternating left-toright and right-to-left passes over the tree by reading the preorder representation, saving the "stalk" of the tree on a stack, and writing out a postorder representation. However, left-to-right postorder is equivalent to right-to-left preorder, and right-to-left postorder is equivalent to left-to-right preorder, which means that at the end of one pass the intermediate file is ready for the next pass in the reverse direction. The maximum amount of primary storage needed for this method is 0 (height of the tree).

It is not apparent that one method is always faster than the other. To use Schulz's technique, the alternating-pass evaluator is unable to skip over subtrees in which no attributes will be evaluated; the entire 
tree must be traversed on each pass. Farrow points out that

- . it is irksome to discover that one pass of the evaluator may be spent doing little but turning the tree around.

[Farrow 1982a]

In contrast, the $\sqrt{n}$-evaluation method is guaranteed to make progress during each subproblem. On the other hand, the $\sqrt{n}$-evaluation method may be inefficient because values of attributes calculated in order to evaluate one distinguished attribute may be thrown away only to be recalculated when it comes time to evaluate some other distinguished attribute. This sort of inefficiency can probably be reduced by clustering subproblems so that several distinguished attributes, rather than just a single distinguished attribute, can be evaluated when a given component is resident in primary storage.

Although it is probably of only minor importance, the alternatingpass evaluator has the drawback that there exist some grammars that cannot be evaluated in any fixed number of passes [Kennedy \& Warren 1976], and so the method is applicable only to a subclass of the noncircular attribute grammars. In contrast, the $\sqrt{n}$-method works for any noncircular grammar.

\subsection{An evaluation algerithm that saves at most $l(\log \mathrm{n})$ values}

This section describes an algorithm for evaluating a distinguished attribute of an n-attribute tree that saves at most $0(\log n)$ attribute values at any stage of evaluation. The algorithm uses a recursive divide-and-conquer strategy; as with the $\sqrt{n}$-evaluation algorithm, the problem is divided into a partitioning step, a projection step, and an 
evaluation step, described in Sections $7.3 .1,7.3 .2$, and 7.3 .3 , respectively •

We will assume that each node in the tree is labeled with its subordinate and superior characteristic graph. The subordinate characteristic graphs can be constructed during a single endorder traversal of the tree; the superior characteristic graphs can then be constructed during a single preorder traversal of the tree. These passes require no more than a linear amount of time and a constant amount of space. Storing the graphs takes no more than MaxAttrs ${ }^{2}$ bits at each tree node.

\subsubsection{Partitioning}

Because the $0(\log n)$-evaluation algorithm is recursive, the partitioning step is applied to a component of the tree, rather than to the entire tree. The goal of the partitioning step is to find a set of nodes that partitions a component into at most MaxSons ${ }^{2}$ subcomponents, each of which contains no more than $\mathrm{m} /$ MaxSons +1 nodes, where $\mathrm{m}$ is the number of nodes in the original component.

To abide by the $O(\log \mathrm{n})$ requirement on the amount of additional space used, the partitioning step cannot use a recursive tree-walk, as is done in the second and third phases of partitioning in the $\sqrt{n}-$ evaluation algorithm. To carry out a recursive tree-walk, it is necessary to have at least as much stack space as the height of the component. In genera1, the height of a component of size $m$ is not related to $\log \mathrm{m}$ by any fixed constant. The partitıoning method described in this section partitions a component using no more than a constant amount of additional storage, at the cost of having a quadratic running time. 
The procedure Partition, stated below as Algorithm 7.6 , constructs a partition set $Q$ bottom-up, by considering each node in an endorder traversal of the component $K$, where $K$ is one of the components detined by the set $P .^{5} Q$ is initially empty, and Partition adds a new node to $Q$

A1goritm 7.6. Given a component $K$ that has $m$ nodes, where $K$ is defined by the set $P$, find a set of nodes that partitions $K$ into no more than MaxSons ${ }^{2}$ components, each containing no more than $\mathrm{m} /$ MaxSons +1 nodes.

Partition(K, P):

let

$K=a$ component of the derivation tree

$P, Q=$ sets of tree nodes

$X=a$ tree node

$\mathrm{m}=$ an integer

in

$Q:=\varnothing$

$\mathrm{m}:=$ the number of nodes in $K$

for each node $X$ in an endorder traversal of $K$ do

if $\operatorname{CountNodes}(X, P, Q)>m / M_{a x S o n s}{ }^{2}$ then

od Insert $X$ into $Q$

$\operatorname{return}(Q)$

CountNodes $(X, P, Q)$ :

let

$X, Y=$ tree nodes

$P, Q=$ sets of nodes

in

Count $=$ an integer

for each node $Y$ in an endorder traversal of the subtree rooted at $X$ that descends no further when encountering a member of $P \cup Q$ do

Count $:=$ Count +1

od

return(Count)

${ }^{5} \mathrm{P}$ represents the context in which a call on Partition is made. $\mathrm{K}$ is a connected region of the tree defined by nodes in $P$; the root of $K$ is in $P$, and some of the leaves of $K$ are also in $P$. For now, it is easier to ignore $P$ altogether, and think of $K$ itself as a tree. 
whenever a node is found that is at the root of a component larger than m/MaxSons ${ }^{2}$. For each node $X$, Partition uses the procedure CountNodes to compute how large a component there would be if $X$ were the root of a component. CountNodes performs an endorder traversal of the subtree rooted at $x$, descending no further when encountering a node that is a member of either $P$ or $Q$. Each child of $X$ contributes from 1 to m/MaxSons ${ }^{2}$ nodes to this total; thus, the size of the component rooted at $\mathrm{X}$ is no larger than $\mathrm{m} /$ MaxSons +1 .

Each call on CountNodes takes up to $O(m)$ steps, and each of the $m$ nodes in the tree causes one call on CountNodes; consequently, the total running time of Algorithm 7.6 is $0\left(\mathrm{~m}^{2}\right)$.

We can show that the number of components found by Algorithm 7.6 is no more than MaxSons ${ }^{2}$ by the following argument: The sum of the sizes of the components $K_{Q}$, defined by the partition $Q$ found by Algorithm 7.6, is related to the total number of nodes $m$ by:

$$
\mathrm{m}=1+\sum_{\mathrm{k} \in \mathrm{K}_{\mathrm{Q}}}(|\mathrm{k}|-1)
$$

Each component, except possibly the one containing the root of the tree, contains at least $\mathrm{m} /$ MaxSons ${ }^{2}+1$ nodes, so

$$
m \geq 1+\left(\left|k_{Q}\right|-1\right)\left(m / M^{2} \text { Sons }{ }^{2}\right)
$$

Solving for $\left|\mathrm{K}_{Q}\right|$, we get:

$$
\begin{aligned}
\left|\mathrm{K}_{Q}\right| & \leq \frac{(\mathrm{m}-1)\left(\text { MaxSons }^{2}\right)}{\mathrm{m}}+1 \\
& \leq \text { MaxSons }^{2}
\end{aligned}
$$


Thus, the partitioning step uses quadratic time to find no more than MaxSons ${ }^{2}$ components, each containing no more than m/MaxSons +1 nodes.

\subsubsection{Projection}

The $O(\log n)$-evaluation algorithm treats each of the attributes of the partition-set nodes found by the partitioning step as the distinguished attribute of a subproblem. As in the $\sqrt{n}$-evaluation algorithm, it is necessary to ensure that the work required to solve each of the subproblems involves only evaluations of attributes that are part of a single component. To do this, the subproblems must be solved in an order that respects the order of the distinguished attributes of the subproblems in the partial order given by the dependency graph $D(T)$.

If $Q$ is the partition set that defines these subproblems, the proper order is found by constructing the graph $G=D(T) / A(Q)$ with the procedure Project, stated as Algorithm 7.7. As with the partitioning step, we do not have enough stack space available to carry out this process with the same method used by the $\sqrt{n}$-evaluation algorithm. (For example, depth-first search of the dependency graph of an m-node component requires more than $O(\log \mathrm{m})$ storage, in general). Thus, Algorithm 7.7 uses a different projection method than the one used in Algorithm 7.4; the method given in Algorithm 7.7 makes use of (MaxAttrs ${ }^{2}$ )(MaxSons ${ }^{4}$ ) bits at each node of the tree, but only a constant amount of other storage.

As is the case with Partition, given in the previous section, the set $P$ represents a context in which Project is called, and defines the component $K, Q$ is a set of nodes that partitions $K$, i.e. all elements 
A1gorithn 7.7. Given a component $K$, where $K$ is defined by the set $P$, and a partition of $K$ named $Q$, build the graph $G=D(T) / A(Q)$.

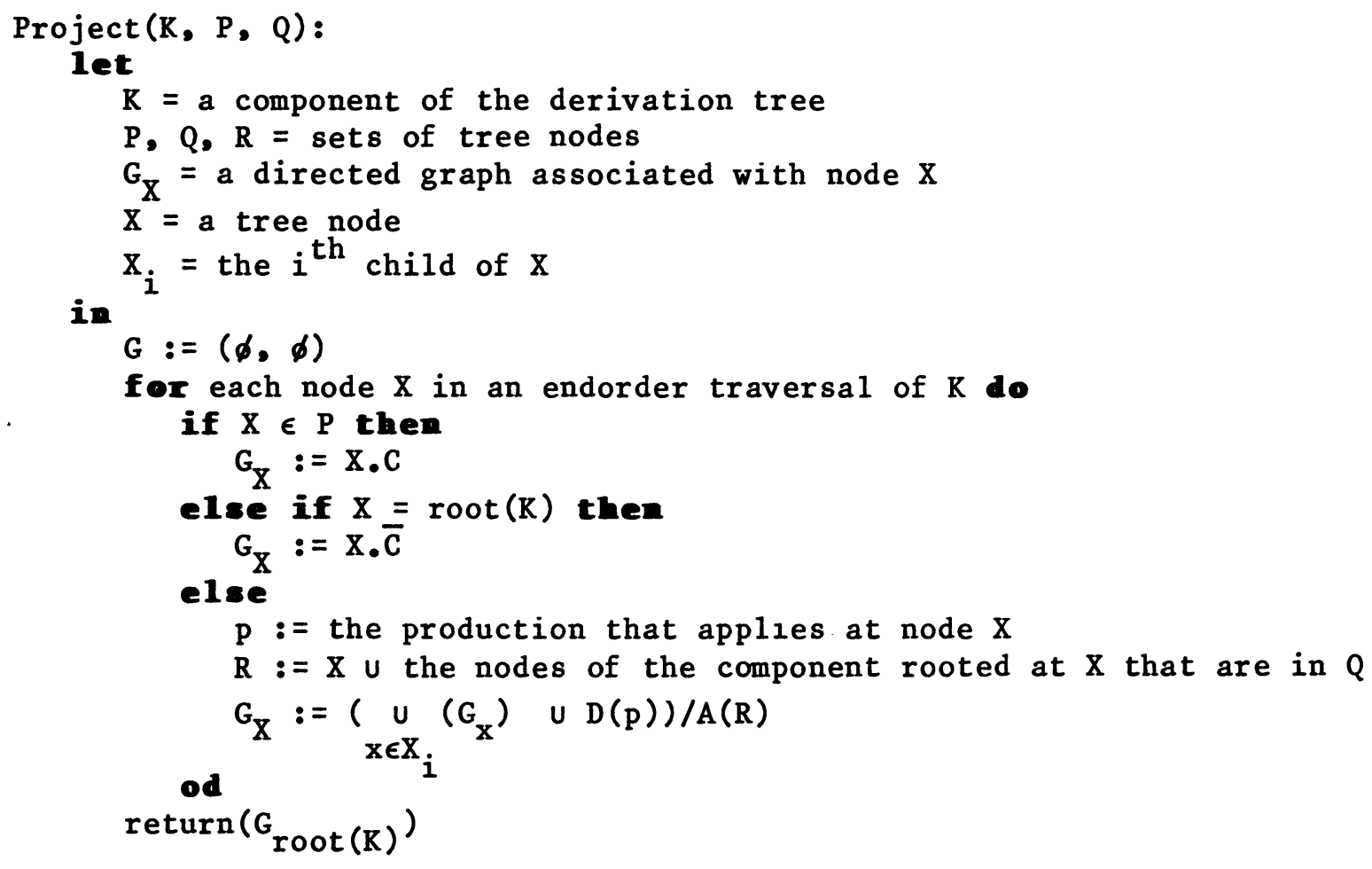

of $Q$ are nodes of $K$. Project constructs $D(T) / A(Q)$ bottom-up, by considering each node of $\mathrm{K}$ in endorder. Let $\mathrm{T}_{\mathrm{X}}$ denote the subtree of $\mathrm{T}$ rooted at $X$. For each node $X$ in $K$, Project computes a graph $G_{X}$ that is the projection of $D\left(T_{X}\right)$ onto $R$, where $R$ is the set $X \cup\{$ all nodes of $Q$ that are in $T_{X}$. Given the graphs $G_{X_{i}}$ for all sons $x_{i}$ of $X_{\text {, the computation }}$ of $G_{X}$ takes no more than a constant amount of time and space.

Because $Q$ is no larger than MaxSons ${ }^{2}, G_{X}$ contains no more than $\left(\right.$ MaxAttrs $\left.{ }^{2}\right)\left(\right.$ MaxSons $\left.^{4}\right)$ vertices. Consequently, $G_{X}$ can be represented 
using a dependency matrix that contains no more than $\left(\right.$ MaxAttrs $\left.{ }^{2}\right)\left(\right.$ MaxSons $\left.^{4}\right)$ bits.

Project makes a single pass over $K$, and does a constant amount of work at each node, so the total running time is $O(|R|)$; its storage requirement is bounded by a constant, plus (MaxAttrs ${ }^{2}$ ) (MaxSons ${ }^{4}$ ) bits at each node of the tree.

\subsubsection{The attribute evaluation algorithm}

The attribute evaluation algorithm stated as Algorithm 7.8 consists of the mutually recursive procedures DivideAndConquer and DemandAncestors. DivideAndConquer solves the problem of evaluating a distinguished attribute $b$ in a component $K$ defined by the partition set $P$. As the name suggests, a divide-and-conquer strategy is used to break the problem up into smaller problems. The method used is first to call the procedure Partition, given as Algorithm 7.6, to find a set of nodes $Q$ that partitions $K$ into smaller components, and then let the attributes of $A(Q)$ be distinguished attributes of smaller problems. By the argument given in Section 7.3 .1 , each of these components is no larger than $|\mathrm{R}| /$ MaxSons +1 .

The argument $P$ in DivideAndConquer is a set of nodes consisting of all nodes found by Partition in active calls to DivideAndConquer. To confine the amount of work to a single one of the components defined by P, whenever DivideAndConquer is called to evaluate attribute $b$, a11 attributes belonging to $A(P)$ that are ancestors of $b$ have values. Initially $P$ is the empty set, so the condition is trivially established by Evaluate. 
Algorithe 7.8. Given a semantic tree $T$ and a distinguished attribute b, evaluate b.

Evaluate $(T, b)$ :

let

$T=$ a derivation tree

$\mathrm{b}=$ an attribute instance

in

DivideAndConquer $(b, \phi)$

DivideAndConquer (b, P)

let

$\mathrm{b}=$ an attribute instance

$P, Q=$ sets of tree nodes

$\mathbf{G}=$ a directed graph

$T=$ a derivation tree (global)

$\mathrm{K}=\mathrm{a}$ component of $\mathrm{T}$

in

$\mathrm{K}:=$ the component of $\mathrm{T}$ defined by $\mathrm{P}$ containing the arguments of $\mathrm{b}$

if $|K| \leq$ MaxSons ${ }^{2}$ then DemandValue(b)

else

$Q:=$ Partition $(K, P) \cup$ TreeNode $(b)$

$G:=\operatorname{Project}(K, P, Q)$

DemandAncestors $(b, G, P \cup Q)$

Release storage for attributes that do not belong to $A(P)$

DemandAncestors $(b, G, P)$ :

let

b, $c=$ attribute instances

$G=a$ directed graph

$P=$ a set of tree nodes

in

While there exists $c$, an unevaluated predecessor of $b$ in $G$ do

DemandAncestors $(c, G, P)$

od

DivideAndConquer $(b, P)$

To establish this condition for the subproblems that result from the call to Partition, DemandAncestors orders the calls to solve subproblems so that they respect the order of attributes in $D(T)$; this is accomplished by passing DemandAncestors the graph $G=D(T) / A(Q)$, found by Project. DemandAncestors causes values to be given to unevaluated ancestors of $\mathrm{b}$ in $\mathrm{G}$ by a recursive demand-evaluation process initiated 
on the unevaluated predecessors of $b$ in $G$. Because $G$ is no larger than (Maxattrs)(MaxSons ${ }^{2}$ ), the number of recursive calls made is bounded by (Maxattrs)(MaxSons ${ }^{2}$ ).

Because each call on Partition divides a component into no more than MaxSons ${ }^{2}$ subcomponents, the number of subproblems is no more than (Maxattrs)(MaxSons ${ }^{2}$ ). Because the problem is subdivided until components are no larger than MaxSons ${ }^{2}$, no more than (Maxattrs)(MaxSons ${ }^{2}$ ) attributes are needed to evaluate the distinguished attribute of a component by the call to DemandValue. Thus, the number of values needed to evaluate a tree of size $m$ is described by the recurrence equation:

$$
\begin{aligned}
& \nabla(m) \leq V\left(\frac{m}{\text { MaxSons }}+1\right)+(\text { MaxAttrs })\left(\text { MaxSons }^{2}\right) \\
& V(m) \leq(\text { MaxAttrs })\left(\text { MaxSons }^{2}\right), \text { for } m \leq \text { MaxSons }^{2}
\end{aligned}
$$

which has the solution $V(m)=0(\log \mathrm{m})$.

The number of steps used by Algorithm 7.8 is described by:

$$
\begin{gathered}
T(m) \leq T\left(\frac{m}{\text { MaxSons }}+1\right)+(b)\left(m^{2}\right) \\
T(m) \leq\left(\text { MaxAttrs) }\left(\text { MaxSons }^{2}\right), \text { for } m \leq \text { MaxSons }^{2}\right.
\end{gathered}
$$

which has the solution $T(m)=0\left(m^{2}\right)$.

\subsubsection{Discussion}

Although for many semantic trees it is possible to evaluate a distinguished attribute using fewer than $0(\log n)$ attribute values, in general a better bound than $O(\log n)$ is impossible, as shown by means of an example. It has been established that pebbling an n-vertex graph that is a complete binary tree with the edges directed towards the root 
requires $\Omega(\log \mathrm{n})$ pebbles [Paterson \& Hewitt 1970]. This establishes a lower bound on the number of attribute values that need to be saved during evaluation, because a derivation tree of the following grammar that is a complete binary tree has a dependency graph that is a complete binary tree with the edges directed towards the root:

$$
\begin{aligned}
& S \rightarrow S \text { S } \\
& s_{1} \cdot s:=f\left(s_{2} \cdot s, S_{3} \cdot s\right) \\
& S \rightarrow \frac{1}{\text { S.S }:=1}
\end{aligned}
$$

For example, the tree:

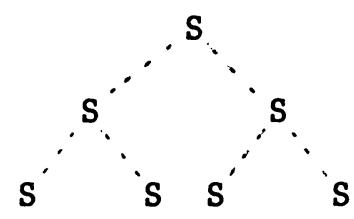

has the dependency graph:

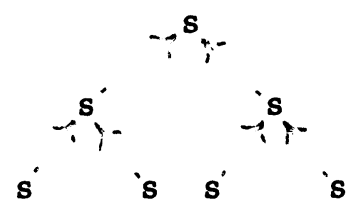

On this example, every evaluation method must use at least $0(\log n)$ attribute values.

The quadratic behavior of Algorithm 7.8 is due to the quadratic running time of the partitioning step, which raises the question of whether it is possible to do better. We can show that only a certain amount of improvement is possible because attribute evaluation by a method that saves no more than $O(\log n)$ values is inherently nonlinear; that is, we can demonstrate the existence of dependency graphs for which using $O(\log n)$ pebbles requires using a non-linear number of steps. 
Swamy and Savage showed that for large $n, \Omega(n \log n)$ steps are required to pebble the upper-left vertex of graphs of the form:

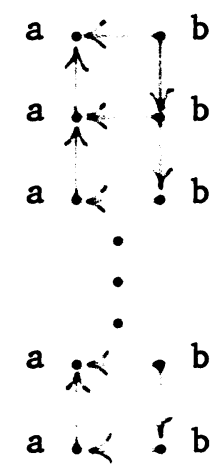

when no more than $O(\log n)$ pebbles are used [Swamy \& Savage 1979]. This establishes that doing attribute evaluation by a method that saves no more than $O(\log n)$ values is inherently nonlinear, because graphs of this form are dependency graphs of the grammar:

$$
\begin{aligned}
& \mathrm{S} \rightarrow \mathrm{S} \\
& \mathrm{s}_{1} \cdot \mathrm{s}:=\mathrm{f}\left(\mathrm{s}_{1} \cdot \mathrm{b}, \mathrm{s}_{2} \cdot \mathrm{a}\right) \\
& s_{2} \cdot b:=g\left(s_{1} \cdot b\right) \\
& \text { s } \quad a^{a} \\
& s: y^{\circ} \\
& S \rightarrow x \\
& \begin{array}{lll} 
& a^{b} \\
\vdots & & \\
\dot{x} & &
\end{array}
\end{aligned}
$$

As presented, the $0(\log n)$ algorithm is probably not a practical evaluation method because of its quadratic running time. However, Algorithm 7.6 only makes use of a constant amount of additional storage to partition a component; one problem for further research is whether by using $O(\log n)$ units of additional storage it is possible to $f$ ind an algorithm for partitioning a component whose running tıme is $0(\mathrm{~m} \log \mathrm{m})$. 
CHAPTER 8

Efficient Representations of Large Attributes

Attribute grammars of ten employ attributes belonging to data types that require a large data structure to represent a single value. For instance, attribute grammars for programming languages commonly use attributes whose values are symbol tables, as is done in Chapter 3 . Design decisions about how to represent values in such data types can greatly affect the efficiency of an attribute-grammar-based system. This chapter is concerned with characteristics of data-type representations that make them suitable for use in attribute-grammar specifications.

\subsection{Sharing common substructures}

If a representation is chosen that makes it necessary to give each attribute instance its own copy of a large structure, it will require an inordinate amount of space to store a semantic tree; furthermore, because each semantic function application will have to create one of these large structures, it will require an inordinate amount of time to evaluate the tree. Thus, both the space needed for storing large value-structures and the time needed for creating them may impose a great deal of overhead on an attribute-grammar-based system. 
This section discusses how to avoid such inefficiencies by designing data-type representations in such a way that elements of the type can share storage in common. This method reduces the storage overhead because the space needed for storing large attribute values is shared among many attribute instances, and it reduces the time overhead because new values of large attributes are constructed from structures that have been previously built up.

Sharing storage among several data-type elements introduces two complications. First, data-type operations must have no side-effects on their arguments, because any change to the internal representation of one value could cause changes to the other values with which it shares substructures in common. Second, storage reclamation in the underlying system is more complicated. The data structure that represents an attribute value cannot be reclaimed blindly, because some of its substructures could still be included as part of the value of one or more other attributes; consequently, storage reclamation must be handled using either garbage collection or a system of reference counts.

It is common practice for large attributes to be accessed through one level of indirection so that a single structure represents the value of several attribute instances that have identical values [Schulz 1976, Răihä 1979, Farrow 1982a]. A second benefit of this strategy is that semantic functions that are identity functions can copy pointers rather than having to copy entire structures.

This strategy can be taken a step further by arranging for the structures that represent nearly identical values to share most of the same substructure in common. When this is done, storing two nearly 
identical values requires only marginally more space than storing just one of the values; semantic functions that return a value nearly identical to their arguments require little time to construct the return value. For example, a symbol table can be represented by a linked list that is accessed through a pointer to the head of the list. A linkedlist representation of a symbol table is a sharable data structure because when the names contained in one table are a subset of the names contained in the other table, both tables can be represented using a single list that has the common names at the tail of the list. A new name can be added to the symbol table by concatenating it to the head of the list. The full set of names is accessed through a pointer to the head of the list; the smaller set of names is accessed through a pointer to the common tail.

The idea of implementing symbol-table attributes with linked-1ists so that storage can be shared in common is not a new one, but to the author's knowledge, linked-lists have been the only sharable structures previously used in attribute-grammar-based systems. The rest of this section describes a technique that can be used to design sharable representations for a wide variety of data types. First we show that a linked-1ist is not the only sharable data structure that can be used to represent a symbol table; then we show how to extend this idea to other data types.

The principle idea is that a 2-3 tree is a sharable representation of an ordered set if the tree is manipulated in a slightly non-standard way. Normally, the elements of the set are associated with the leaves of the tree in increasing order from left to right, and each interior 
node is labeled with five items: the addresses of its two or three sons, the value of the largest element stored in the subtree rooted at the leftmost son, and the value of the largest element stored in the subtree rooted at the middle son [Aho et a1. 1974]. During the course of such operations as INSERT, DELETE, MIN, SPLIT, and CONCATENATE, the tree may be restructured, in which case these fields take on new values.

We define a sharable 2-3 tree to be a standard 2-3 tree on which all operations are carried out in the usual fashion, except that whenever one of the fields of an interior node $M$ would normally be changed, we create a new node $N$ that duplicates $M$, and we change the field in $N$. of course, to be able to treat $N$ as the son of parent( $M)$, it is necessary to change the appropriate son-field in parent(M), so we create a new node that duplicates parent $(M)$, and so on, all the way to the root of the tree. Thus, new nodes are introduced for each of the original nodes along the path from $M$ to the root of the tree. Because an operation that restructures a standard 2-3 tree may modify all of the nodes on the path to the root anyway, and because a single operation on a standard 2-3 tree that has $n$ nodes takes at most $0(\log n)$ steps, the same operation on a sharable 2-3 tree introduces at most $0(\log \mathrm{n})$ additional nodes and also takes at most $0(\log n)$ steps. The new tree resulting from the operation shares the entire structure of the original tree except for the nodes on a path from $N$ to the root, plus at most $O(\log n)$ other nodes that may be introduced in order to maintain the 2-3 property. For example, the effect of an INSERT operation is illustrated in Figure 8.1; in this example, no rebalancing needs to be done. 


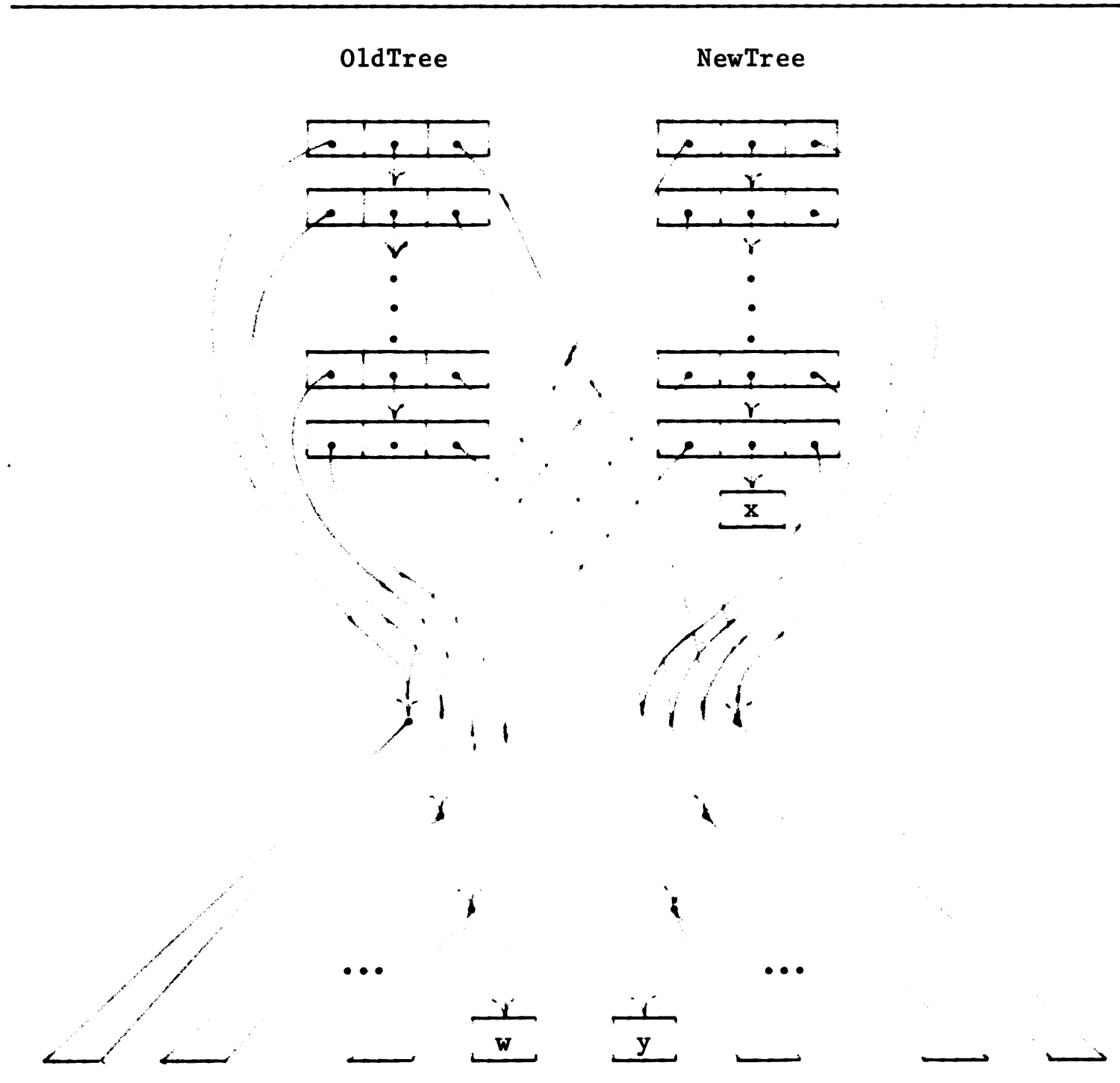

Figere 8.1. The effect of the operation NewTree := INSERT(x, 01dTree), showing how common substructures are shared in a sharable 2-3 tree.

Sharable 2-3 trees are very versatile structures that can be used to implement efficient INSERT, DELETE, MEMBER, MIN, SPLIT, and CONCATENATE operations on ordered sets. For symbol tables, a sharable 2-3 tree representation is better than a linked-1ist representation because MEMBERship tests and INSERT operations on an n-entry symbol table 
require at most $0(\log n)$ steps with a sharable 2-3 tree, but $O(n)$ steps (expected time) with a linked-1ist representation.

Sharable 2-3 trees can also be used to design sharable representations for a variety of other data types. For example, an array can by implemented using a sharable 2-3 tree as a dictionary in which the keyvalue for a leaf is its index in the array. All array operations can be defined as a sequence of the operations UPDATE and LOOKUP, where UPDATE is an $0(\log n)$ operation for modifyıng the information stored at a given key-value (as before, this involves rebuilding the spine of the dictionary), and LOOKUP is an $O(\log n)$ operation that returns the information stored in a cell with a given key-value.

In one sense, sharable 2-3 trees provide a universal technique for making any data-type representation a sharable structure, at the cost of making each data-type operation run slower by a log factor. The data structures used for any data-type representation can be embedded in an array; in principle, this array can be represented by a sharable 2-3 tree. However, this technique is not likely to produce satisfactory results because the slow-down factor is proportional to the logarithm of the size of the tree; in this case, the tree encompasses the total amount of storage used to represent the existing elements of the type. In contrast, when sharable 2-3 trees are used to represent symbol tables, each tree represents an individual symbol table.

A general limitation on the sharable 2-3 tree technique should be noted; although it is true that "nearby" values will share common substructures, "nearby" is defined in a "computation-oriented" rather than a "value-oriented" fashion. For example, two sets $A$ and $B$, with values 
$\{a, b, c, d\}$ and $\{b, c, d, e\}$, respectively, will be able to share some common substructures only if the value of $A$ was used in computing the value of $B$, or vice versa. Even if $A$ is used in computing the value of B, they still might not share any substructures in common because there could be a complete turnover of nodes by the time $B$ is constructed, due to a long sequence of intervening operations.

\subsection{Rapid testing of equality}

When change propagation is employed to update a semantic tree, the new attribute value computed by each semantic function application is compared to the old attribute value to see if changes need to be propagated further. Consequently, using a slow method for testing equality of large attributes will impose a large amount of overhead on the system, and will make change propagation be very inefficient. However, if each of the data-type elements has a unique representation, two elements can be tested for equality by comparing two pointers; furthermore, the test takes unit-time regardless of whether or not the elements are equa1.

For example, trees constructed with a hashed-cons function are defined uniquely, and two trees can be tested for equality by comparing the addresses of their roots [Goto 1974]. Unit-time equality-testing is also possible for other data types; for example, Sassa and Goto describe a representation for sets that uses a hash-coding technique to guarantee that sets are represented uniquely [Sassa \& Goto 1976]. This representation has a unit-time equality test, as well as a unit-time MEMBERship test. 
Some data-type representations allow "nearby" elements to share common substructures, and other representations allow two elements to be tested for equality in unit-time. In general, we do not know how to provide both of these properties in a single representation. Although trees constructed with a hashed-cons function have a fast equality test and also have the property that nearby elements share common substructures, it is not true that the hashed-cons operation can be used to provide automatic, unit-time equality tests for arbitrary data types; enforcing unique structures is not the same thing as enforcing unique representations. For instance, the set $\{a, b, c, d, e\}$ can be represented by either of the following two 2-3 trees:

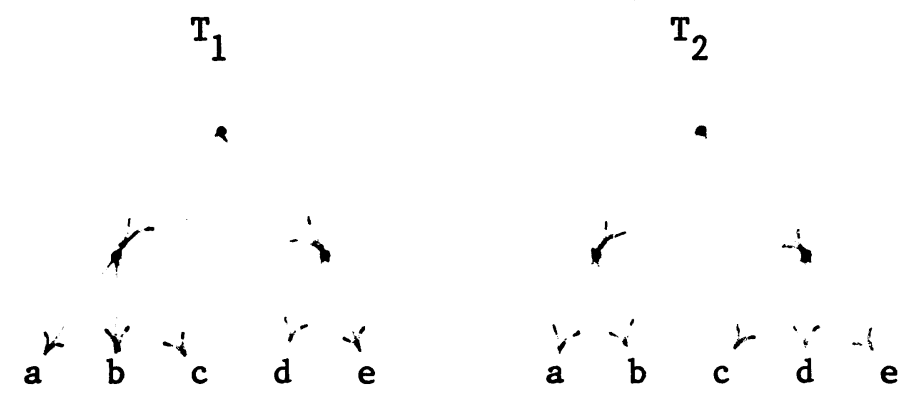

Even when $T_{1}$ and $T_{2}$ are constructed with a hashed-cons function, the addresses of their roots will not match.

\subsection{Discussion}

One technique that can be used to design sharable data-type representations is to implement parts of the representation structure with sharable 2-3 trees. For example, as described in [Sassa \& Goto 1976], hashed sets that have nearly identical values do not share any storage in common, but by reimplementing certain array structures used in the representation with a sharable 2-3 tree, nearly identical sets 
can share most of the same storage, at the cost of increasing the time to test MEMBERship to $O(\log n)$.

Sharing storage and rapid testing of equality may be just two factors influencing the choice of representation; for example, the efficiency of the insertion operation and the lookup operation of a symboltable data type can be just as important to the overall efficiency of the system as sharing and equality-testing. The goal is to be able to increase the amount of sharing and to decrease the time needed to test equality while imposing as little overhead as possible on the data-type operations, so that semantic-function applications are no more timeconsuming than necessary.

One technique that may be valuable for optimizing a system's performance is to use different representations of a data type in different parts of the grammar. To illustrate this, consider an attribute grammar for a programming language that detects undeclared variables as well as multiply-declared variables. As in the example given in Chapter 3, a symbol table would be built up in the declarations section of each block and then passed to every statement of the block by means of copy rules. Among the declarations, INSERT operations are carried out to construct the symbol table and MEMBERship tests are carried out to detect multiply-declared variables, while among the statements only MEMBERship tests are needed. Thus, it would be advantageous to use sharable 2-3 trees to represent symbol-tables of declaration nonterminals and to use hashed sets to represent symbol-tables of statement nonterminals. The translation between representations would be made in the production for a block, where the symbol table received from the declaration list is 
passed to the statement list; that is, the production for a block would be something like:

\section{Block $\rightarrow$ begin Decllist StmtList end StmtList.used := DefineHashedSet(DeclList.updated)}

The topic of designing data-type representations that are suitable for use in attribute-grammar specifications is one that deserves further study. This chapter has discussed criteria for what makes a data-type representation suitable for use in attribute-grammar specifications, but it has not presented techniques that can be applied automatically by a compiler-compiler or an editor-generator. The responsibility for choosing an efficient representation is entirely in the hands of the person who defines the type -- either the system-designer defining one of the primitive types of the system, or someone who is writing an attributegrammar specification, such as an editor-designer, using a typedefinition facility of the system. Sharing occurs only when a sharable data structure is chosen and when the semantic functions cause storage to be shared; rapid testing of equality is only possible when a fast equality test exists for the data-type representation. 
CHAPTER 9

Incremental Evaluation for Restricted Classes of Attribute Grammars

The optimal-time algorithm for incremental attribute evaluation presented in Chapter 5 has the advantage of being applicable to arbitrary noncircular attribute grammars; unfortunately, its generality is achieved at the expense of storage economy. Not only are semanticfunction applications scheduled according to an attribute's position in a graph structure built during the updating process, but each node in the semantic tree must be labeled with either its superior or subordinate characteristic graph. In practice, the storage used for these graphs dominates the storage for the attribute values. It would be desirable to eliminate the need for these graphs, yet retain the ability to make optima1-time updates to the tree.

One line of research on non-incremental evaluation has been to investigate algorithms for subclasses of attribute grammars, defined by placing restrictions on the patterns of dependencies allowed in the semantic equations. A variety of special evaluation algorithms have been devised that take advantage of such restrictions. It is worthwhile to pursue this idea in the context of incremental evaluation also, with the goal of finding an attribute-grammar subclass for which there is an optimal-time updating algorithm that does not require as much storage overhead as the method described in Chapter 5. This chapter discusses 
optimal-time updating algorithms for four attribute-grammar subclasses that have previously been defined in the literature: L-attributed, bottom-up/top-down, absolutely noncircular, and ordered attribute grammars.

9.1. Incremental evaluation for two simple classes

For certain attribute-grammar subclasses, there are very simple optımal-time algorithms for updating a tree after a subtree replacement. One example is the class of L-attributed grammars, which formalize semantic processing in top-down parsers; an attribute grammar is Lattributed if for each production $p: x_{0} \rightarrow x_{1} \cdot \cdots x_{n}$, every argument of an inherited attribute of a right-side symbol $x_{k}$ is either an inherited attribute of $x_{0}$ or a synthesized attribute of a right-side symbol $x_{i}$ that appears to the left of $x_{k}$ in $p$, i.e. $1 \leq i \leq k$ [Lewis et al. 1974]. The attributes of an L-attributed grammar can be evaluated during a left-to-right traversal of the semantic tree. To update a semantic tree of an L-attributed grammar after a subtree replacement at node $r$, carry out a left-to-right traversal starting at node $r$, descend no further when attributes no longer change value, and halt upon finding an ancestor of $r$ whose attributes do not change value.

A second example of a subclass for which there exists a simple updating algorithm is the class of grammars that can be evaluated during a bottom-up pass over the tree followed by a top-down pass over the tree. This class is an important subclass because many global data-flow problems can be expressed with this kind of grammar [Farrow 1977, Rosen 1980]. To update a tree following a subtree replacement at node $r$, 
start at $r$ and perform evaluations in bottom-up order until attributes no longer change value, then reverse the direction of the traversal, and perform evaluations in top-down order until attributes no longer change value.

9.2. Incremental evaluation for absolutely noncircular attribute grammars

This section discusses how to perform incremental evaluation for absolutely noncircular attribute grammars. These grammars are a large attribute-grammar subclass that were introduced in [Kennedy \& Warren 1976] to simplify the construction of a distributed attribute evaluator, an evaluator that uses a collection of finite state machines to control the evaluation process. This section describes how a distributed evaluator can be used for incremental attribute evaluation; an optimaltime updating algorithm is presented that takes exactly the same machines produced by the Kennedy and Warren construction, and uses them for change propagation by giving an alternative interpretation to the instructions in the machines' finite state control.

Distributed attribute evaluation can be viewed as an improvement on the greedy evaluation method described in the next section.

\subsubsection{Greedy evaluation}

Because attributes are defined strictly in terms of other attributes in a single production, when one attribute instance is evaluated, other attribute instances in the same production instance are also apt to be ready for evaluation; consequently, an evaluator can reduce the number of times it has to switch to a new evaluation context by updating 
as many attributes as possible at one production instance before moving on to the next. Unlike the evaluation methods described in earlier chapters, the greedy evaluation method attempts to cluster attribute evaluations in this way. The method is asymptotically neither better nor worse than the methods given earlier, but in practice it can be better if switching to a new evaluation context is an expensive operation.

To further characterize partially attributed semantic trees, we introduce the notion of deficiency; we say that a production instance $p$ is deficient if any of the attribute instances of $p$ are ready for evaluation. We define update $\mathfrak{R}$ to be the operation that evaluates all attribute instances of $p$ that are ready for evaluation.

Because attributes are defined solely in terms of attributes of a single production, the only production instances that can become deficient when production instance $\mathrm{p}: \mathrm{x}_{0} \rightarrow \mathrm{x}_{1} \cdots \mathrm{x}_{\mathrm{n}}$ is updated are the neighbors of $R$, that is, the production instance that derives $x_{0}$ and the

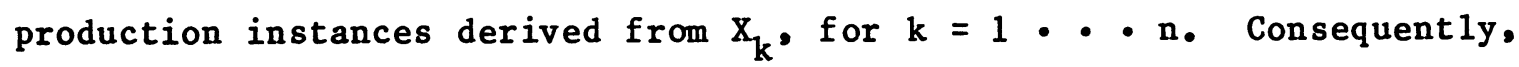
a greedy evaluation algorithm can keep track of all deficient production instances in a work-list, as is done in Algorithm 9.1.

The greedy evaluation method of Algorithm 9.1 has the drawback that the attributes of a production instance $p$ are repeatedly searched to discover which of them are ready for evaluation; in the statement of the algorithm as given, the searches are concealed in the operations to test whether $p$ is deficient and to update p. These searches are carried out whenever the evaluator switches to a new context, and thus a production 
Algorithm 9.1. Attribute evaluation. EVALUATE(T):

let

$T=$ an unevaluated semantic tree

$S=a$ set of production instances

in

$\mathrm{p}, \mathrm{p}^{\prime}=$ production instances

$S:=$ the set of deficient production instances of $T$

vhile $S \neq \varnothing$ do

Select and remove a production instance $p$ from $S$ update $p$

for each production instance $p^{\prime}$ that is a neighbor of $p$ do if $\mathrm{p}^{\prime}$ is deficient then Insert $\mathrm{p}^{\prime}$ into $\mathrm{S}$ od

od

instance will be searched each time it or one of its neighbors is updated by the evaluator.

\subsubsection{Tree-walk evaluation}

Distributed evaluators elıminate the need for this kind of searching at run-time by keeping track of the evaluation situation at each production instance and using scheduling information generated during a one-time analysis of attribute dependencies in the grammar. This information takes the form of a collection of acyclic, deterministic finitestate machines, one for each production in the grammar. A machine state corresponds to a run-time situation in which a particular set of the production's attributes are available; a transition represents either an instruction to evaluate attributes or an instruction to transfer control to another machine.

If $\mathrm{p}: \mathrm{x}_{0} \rightarrow \mathrm{x}_{1} \cdot \cdots \mathrm{x}_{\mathrm{n}}$ is a production, and $\mathrm{t}_{0}$ is a node of type $\mathrm{x}_{0}$ with sons $t_{1} \cdots t_{n}$ of type $x_{1} \cdots \cdot x_{n}$, respectively, we say that 
2 applies at node $t$. For brevity the machine for the production that applies at node $t$ will be referred to as the machine that applies at $t$.

A distributed evaluator is made up of the machines that apply at each node of the tree to be evaluated. At any given moment during evaluation, a single machine of the distributed evaluator is active. Initially, the active machine is the machine that applies at the root of the tree; during the course of evaluation, control passes from machine to machine until all machines reach their final state, at which point the tree is fully attributed. It is not necessary to provide an actual copy of the machine for each production instance; it is sufficient to label each production instance with its state in the appropriate machine.

The distributed evaluation technique is tıme-efficient because, instead of computing the evaluation order at run-time, the evaluator uses a strategy that was pre-computed at construction-time by statically analyzing the grammar. The distributed evaluation technique is spaceefficient because at run-time it uses no large data structures, such as the dependency graph and the worklist used by the evaluators described earlier.

Three varieties of distributed evaluators have been discussed in the literature: tree-walk evaluators [Warren 1975, Kennedy \& Warren 1976], coroutine evaluators [Warren 1976], and local-control automata [Cohen \& Harry 1979], and each of these techniques can be adapted to handle arbitrary noncircular attribute grammars. In general, however, for a distributed evaluator to work in linear-time for an arbitrary noncircular grammar, each tree node must be labeled with its subordinate 
characteristic graph, which is used as an additıonal argument to the machines' transition function [Warren 1975, Cohen \& Harry 1979]. Because we are interested in saving space by doing away with such graphs, this chapter is only concerned with tree-walk evaluators for absolutely noncircular attribute grammars, which do not require such parameterization.

The use of the term "distributed evaluator" is not meant to imply that the attributes of a semantic tree are evaluated in parallel on separate processors; on the contrary, a semantic tree is evaluated by a single procedure that interprets descriptions of the machines that make up the distributed evaluator for the tree. A treewalk evaluator has a particularly simple structure that allows the evaluation procedure to be a recursive tree-traversal routine. When a node $t$ is visited, a plan, or sequence of instructions, is executed, and then control returns to the parent of $t$. An instruction either calls for the evaluation of a synthesized attribute of $t$ or an inherited attribute of a child of $t$, or else an instruction recursively calls for a visit to a child of $t$.

When control is transferred to node $t$ by a recursive call, a plan is selected on the basis of two pieces of information: the current state of $t$, denoted $t$.Machinestate, and the current-input-set, a set of inherited attributes that have become available since the last visit to $t$. Machine states are of two types, entry states and quiescent states, and each machine has a distinguished quiescent state called its initial state. Each time a visit to $t$ is initiated, the machine that applies at $t$ is awakened from a quiescent state $q$ by selecting an entry state $e$ as a function of $q$ and the current-input-set $I$. The machine then executes 
the plan for e, which may initiate deeper visits. On executing the final instruction of the plan, $t$ enters quiescent state $q^{\prime}$, and passes control back to its parent. During the course of evaluation, each node may be visited several times, successive visits bringing additional inherited attributes; thus, the sequence of instructions executed at a node is the concatenation of several plans.

A tree-walk evaluator can be encoded in two tables: GOTO and PLAN, where GOTO is a table of entry states as a function of quiescent state and current-input-set, and PLAN is a table of plans as a function of entry state. When control is transferred to machine $t$ in quiescent state $q$ with current-input-set $I$, the entry state is selected by:

$$
\text { t.MachineState }:=\operatorname{GOTO}(t \cdot \text { MachineState, I })
$$

and the plan for this state is:

$$
\operatorname{PLAN}(t . M a c h i n e S t a t e)
$$

The $i^{\text {th }}$ instruction of this plan will be denoted by:

$$
\operatorname{PLAN}(t \text {.MachineState) (i) }
$$

A plan for production $x_{0} \rightarrow x_{1} \cdots x_{n}$ is made up of three types of instructions :

a) An $\operatorname{EVAL}(k, b)$ instruction causes attribute instance $x_{k} \cdot b$ to be evaluated. Note that for $k=0, b$ must be a synthesized attribute, but for $k=1$ - $n, b$ must be an inherited attribute. 
b) $\quad A \operatorname{VISIT}(k, I)$ instruction for $k=1 \cdots$ - n causes control to be transferred to the machine that applies at $x_{k}$ by calling the treewalk evaluator recursively with arguments $x_{k}$ and current-input-set I.

c) A SUSPEND(q) instruction causes the machine to be placed in quiescent state $q$, and returns control to the the parent of $x_{0} \cdot$ Each plan $P$ has exactly one SUSPEND instruction, the final instruction of the plan, denoted last(P).

A tree with root $r$ is evaluated by initializing each node $t$ to its initial state, and then calling $\operatorname{EVALUATE}(r, \phi)$, where EVALUATE is given by Algorithm 9.2.

\subsubsection{Constructing a tree-walk evaluator}

A tree-walk evaluator does not need to consider attribute dependencies at run-time because the work has been done once and for all at construction-time. The Kennedy-Warren construction method simulates run-time situations and, by analyzing attribute dependencies, builds an evaluation plan for each situation. The construction method ensures that at run-time an EVAL instruction is only executed when all arguments to the appropriate semantic function have been previously evaluated. The algorithm for incremental evaluation that will be discussed in the next section exploits certain other properties of the plan that are also consequences of the construction method.

Creating a complete set of plans involves finding all situations that can possibly occur during evaluation and making a plan for each of them. A complete description of this process can be found in [Warren 
A1gorith 9.2. Tree-walk evaluation procedure (Kennedy and Warren).

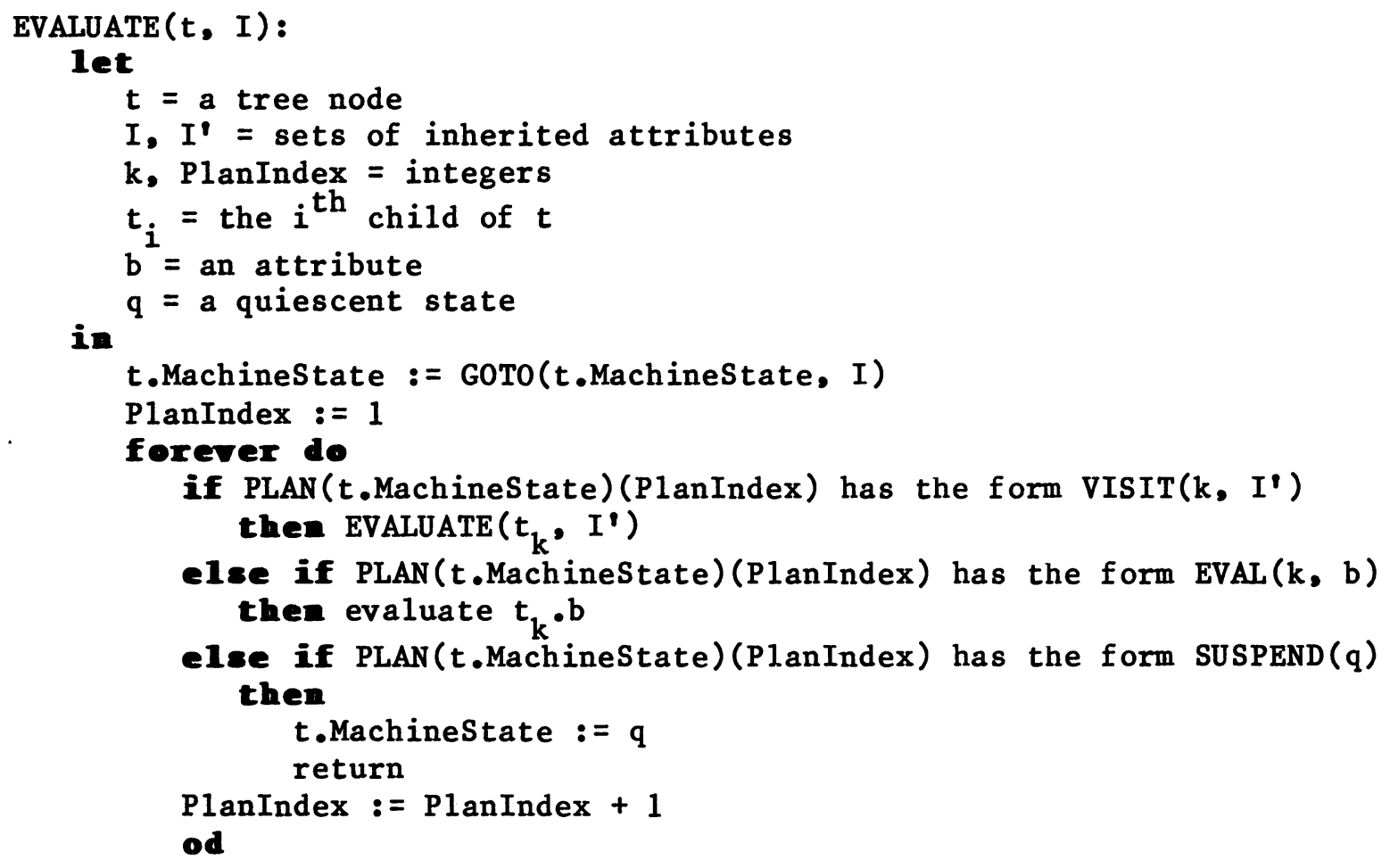

1975] or [Kennedy \& Warren 1976]; however, most of this material is omitted here because an understanding of the details of the construction is not required for understanding the incremental evaluation algorithm. This section describes only the part of the construction concerned with scheduling instructions in a plan for an evaluation situation.

During the construction of a tree-walk evaluator, run-time situations are represented as evaluation states, pairs of the form (,$A$ ), where $\mathrm{p}$ is a production, and $\mathrm{A}$ is a set of attributes of $\mathrm{p}$. An evaluation state $(p, A)$ represents a situation in which the members of $A$ are known to be available at a node where $p$ applies. For each evaluation state, a plan is constructed by repeatedly selecting an instruction 
whose action is permitted under the known dependency constraints, scheduling it as the next instruction in the plan, and then determining what evaluation state results after the instruction terminates.

Given an evaluation state, instructions are scheduled according to the following criteria:

a) An EVAL instruction is scheduled for an attribute that is ready for evaluation in the given state.

b) A VISIT instruction to a child $\mathrm{X}$ is scheduled when at least one inherited attribute of $X$ has been made available since the last visit, and the attribute dependencies ensure that the visit will yield at least one more synthesized attribute of $x$.

c) A SUSPEND instruction is scheduled when no further actions are possible.

To ensure that a tree gets completely evaluated, we extend the definition of attribute grammars by giving each nonterminal of the grammar a dummy synthesized attribute, called the completion attribute. For each production $p: x_{0} \rightarrow x_{1} \cdots x_{n}$, the completion attribute of $x_{0}$ depends on every inherited attribute of $x_{0}$ and every synthesized attribute of each of the right-side symbols $x_{1} \cdots x_{n}$. The completion attributes are simply a device to ensure that the tree-walk evaluator evaluates every attribute in the tree; completion attributes are never actually evaluated at run-time.

EVAL instructions can be scheduled just from the information contained in an evaluation state; scheduling VISIT instructions requires additional information besides the evaluation state. To determine the 
synthesized attributes that will be available after a VISIT to a nonterminal $X$ terminates, it is necessary to know about transitive dependencies of synthesized attributes of $X$ on inherited attributes of $X$; that is, it is necessary to know about the subordinate characteristic graph of $\mathrm{x}$.

Unfortunately, a given nonterminal may have a whole collection of different possible subordinate characteristic graphs. The KennedyWarren construction side-steps this problem by making a worst-case assumption about the subordinate dependencies. Let the set of all possible subordinate characteristic graphs of nonterminal $X$ be denoted $C(X)$. For scheduling purposes, the assumption is made that the subordinate dependencies of $X$ are represented by the graph $P(X)$ constructed by superimposing all the members of $\mathrm{C}(\mathrm{X})$ :

$$
\mathrm{P}(\mathrm{X}):=\underset{\mathrm{c} \in \mathrm{C}(\mathrm{X})}{\mathrm{U})} \mathrm{c}
$$

Each edge in $P(X)$ represents a transitive dependency in at least one subtree that may be derived from $X$, but $P(X)$ may be over-pessimistic in that certain combinations of dependencies may never appear in any one subtree derived from $X$.

One consequence of this assumption is that an evaluation state ( $p, A)$ used during the construction of the evaluator has only an approximate record of the synthesized attributes that at run-time are actually available at the nonterminals of the right-side of p. At run-time, a VISIT to a child C may cause synthesized attributes to be evaluated that the planning algorithm did not expect would be available until after a subsequent VISIT. This causes no problems at run-time because the plan 
for $p$ simply ignores the fact that they are available; there is less work to do during a later VISIT to $C$, but no EVALs are duplicated in the machine that applies at $C$ because the plan for $C$ does take into account all of C's available synthesized attributes.

A second consequence of representing subordinate dependencies by superimposing subordinate characteristic graphs is that it causes the Kennedy-Warren construction to be unable to build an evaluator for some grammars. The Rennedy-Warren construction only applies to the restricted class of attribute grammars defined as follows: an attribute grammar is absolutely noncircular if for each production $\mathrm{p}: \mathrm{X} \rightarrow \mathrm{x}_{1} \cdots \mathrm{X}_{\mathrm{k}}$, the $\operatorname{graph} \mathrm{D}(\mathrm{p}) \cup \mathrm{P}\left(\mathrm{x}_{1}\right) \cup \cdots \cdot \mathrm{U}\left(\mathrm{X}_{\mathrm{k}}\right)$ is acyclic. ${ }^{1}$ This restriction is probably not a great handicap because reasonable grammars are likely to be absolutely noncircular. Kennedy and Warren present the following argument about why it is reasonable to require that the action taken at a node be independent of its children's subtrees:

This assumption is motivated by the conception of grammar nonterminals as abstractions with definite interpretations of their own; the idea of locally-defined semantics suggests that it should be possible to understand the semantics of a construct without any further knowledge of the abstractions it is built of.

[Kennedy \& Warren 1976]

A third consequence of representing subordinate dependencies by superimposing subordinate characteristic graphs is that the actions of

${ }^{1}$ A more restricted definition, based on Knuth's original, erroneous circularity test [Knuth 1968b], is used in [Kennedy \& Warren 1976], although their construction is actually valid for any grammar that meets the definition given above. 
the tree-walk evaluation machines are independent of the subtrees actually derived from the child nodes. This property is extremely important to the optimal-time behavior of the change propagation algorithm described in the next section.

9.2.4. An optimal-time algorithm for updating attributes by treeralking

Th1s section describes how the tree-walk evaluation method can be used for incremental attribute evaluation; we demonstrate how the tables of a tree-walk evaluator can be used to determine the reevaluation order for a change propagation algorithm that updates a tree in O(|AFFECTED|) steps after a subtree replacement.

When a semantic tree $\mathrm{T}$ is (non-incrementally) evaluated by a treewalk evaluator, the order of the EVAL instructions respects the topological order of $D(T)$; for each attribute $b$, the instruction to evaluate $b$ is preceded by instructions to evaluate all of the arguments of $b$. The tree-walk evaluator implicitly defines a total order on the attributes of $T$ that respects the partial order on the attributes in $T$ given by $D(T)$; we call this order the tree-walk evaluation order of $T$.

The basic idea behind the updating algorithm described in this section is that by scheduling attribute reevaluations in an order that respects the the tree-walk evaluation order, we can ensure that all attributes have consistent values on termination. By ignoring VISIT instructions and SUSPEND instructions under certain conditions, we can skip, in unit-tıme, having to reevaluate an arbitrarily large number of attributes that cannot possibly change value. 
The tree-walk evaluation procedure given earlier as Algorithm 9.2 is a recursive routine that begins by activating the machine at the root of the tree. This formulation of tree-walk evaluation is not suited for attribute updating because when updating begins, the only attributes that are inconsistent are attributes of the point of subtree replacement, which may be nested arbitrarily deeply in the tree. For an incremental evaluation algorithm to work in optimal-time, it must do no more than a constant amount of work before it begins to reevaluate attributes of the point of subtree replacement.

The change propagation algorithm is therefore based on Algorithm 9.3, a restatement of Algorithm 9.2 that does not use recursion. In Algorithm 9.2, the state of the active machine is determined by the value of the node's Machinestate field and the value of the variable PlanIndex. When a VISIT instruction causes a transfer of control in Algorithm 9.2, the value of PlanIndex is implicitly stored in the recursion stack. By contrast, in Algorithm 9.3, the PlanIndex component of the machine at each node $t$ is explicitly stored at the node as t.PlanIndex.

One way the change propagation algorithm extends Algorithm 9.3 is that it makes use of a special set of machines, named Reactivated. At all times during updating, the active machine must be a member of Reactivated. Reevaluations are scheduled according to the EVAL instructions of members of Reactivated, but any VISIT instruction or SUSPEND instruction is ignored if it would transfer control to a machine that is not a member of Reactivated. Ignoring such instructions allows the algorithm 
A1gorithn 9.3. Tree-walk evaluation without recursion. $\operatorname{EVALUATE}(T)$ :

let

$t=$ the node of $T$ whose machine is currently active

$I=a$ set of inherited attributes

$k=$ an integer

$t_{i}=$ the $i^{t h}$ child of $t$

$b=$ an attribute

in

$q=a$ quiescent state

Initialize all machines to their initral state

$t:=\operatorname{root}(T)$

t.MachineState $:=\operatorname{GOTO}(t \cdot$ MachineState, $\phi)$

t.PlanIndex : $=1$

forever do

if PLAN( $t$.MachineState)( $t . P l a n I n d e x)$ has the form VISIT(k, I)

then

$t:=t_{k}$

t.MachineState $:=\operatorname{GOTO}(t \cdot$ MachineState, I)

t.PlanIndex $:=1$

else if $\operatorname{PLAN}(t$. MachineState)( $t . P$ lanIndex) has the form $\operatorname{EVAL}(k, b)$

then

evaluate $t_{k} \cdot b$

t.PlanIndex : = t.PlanIndex +1

else if $\operatorname{PLAN}(t . M a c h i n e S t a t e)(t . P 1$ anIndex $)$ has the form $\operatorname{SuSPEND}(q)$

then

if $t=\operatorname{root}(T)$ then return

else

t.Machinestate $:=q$

$t:=$ parent $(t)$

t.PlanIndex $:=t . P l a n I n d e x+1$

od

to skip over, in unit-time, arbitrarily large sections of the tree in which attribute values cannot possibly change.

As with the updating algorithm presented in Chapter 5, the secret to optimal-time behavior is to keep the size of the data-structure used for scheduling reevaluations proportional to the number of attributes that have received new values since updating began. Thus, Reactivated 
initially consists of the single machine that applies at the point of subtree replacement; a new machine is added to Reactivated only when a new member of AFFECTED is identified.

To be able to reevaluate attributes in tree-walk evaluation order, whenever a new machine is added to Reactivated, it is necessary to establish its MachineState and PlanIndex as if its instructions had been executed all along. The method for doing this is based on the notion of an entry sequence: an entry sequence of node $t$ in tree $T$ is an ordered list of the entry states of the machine applying at $t$, listed in the order they occur in the tree-walk evaluation order of $T$.

Because the Kennedy-Warren construction method uses a worst-case representation of subordinate attribute dependencies, the run-time actions of the machine applying at node $t$ depend in no way on the actual subtrees derived from the children of $t$. Consequently, the sequence of instructions executed at $t$ depends entirely on $t^{\prime} s$ ancestors and is independent of all other nodes in the tree. This property allows us to construct entry sequences by the following recursive method:

a) If $i$ denotes the initial state of the root of tree $T$, the entry sequence of the root is the singleton 1 ist:

$$
<\operatorname{GoTO}(i, \phi)>
$$

b) Given the entry sequence at node $A$, the entry sequence for the $k^{\text {th }}$ child $B$ of $A$ is built by simulating the sequence of instructions that are executed at A during (non-incremental) evaluation. For each $\operatorname{VISIT}(k, I)$ instruction, the appropriate entry state is appended to the entry sequence for $B$. 
Step b) is spelled out in complete detail by the procedure BuildEntrySequence, given as Algorithm 9.4. Because the execution simulation carried out by BuildentrySequence is limited to the activity of a single machine, the time needed to construct an entry sequence is constant for a given grammar.

Entry sequences play a crucial role in the change propagation algorithm based on tree-walking. Updating begins at the node $r$ that is the point of subtree replacement. To carry out reevaluations in an order that respects the tree-walk evaluation order of $T$, the machine that applies at $r$ must be reactivated in the first state in which it is

Algorithn 9.4. Build the entry sequence for the $k^{\text {th }}$ child of a node.

BuildEntrySequence (Parent, Sequence0fParent, $k$ ):

let

Parent $=$ a tree node

Sequence $0 f$ Parent, SequenceOfChild = lists of entry states

i, $\mathrm{j}, \mathrm{k}=$ integers

$I=a$ set of inherited attributes

plan $=$ a list of instructions

EntryState0fChild = an entry state

in

QuiescentStateofChild = a quiescent state

QuiescentStateofChild $:=$ the initial state of the $k^{\text {th }}$ child of Parent EntrySequenceOfChild : = <>

for $i:=1$ to length(Sequence0f Parent) do

plan $:=$ PLAN (SequenceOf Parent $(i)$ )

for $j:=1$ to length(plan) do

if $p \operatorname{lan}(j)$ has the form VISIT(k, I) then

EntryStateOfChild := GOTO(QuiescentState0fChild, I)

Append EntryState0fChild to the tail of EntrySequence0fChild

QuiescentStateofChild := the quiescent state associated with

od

the SUSPEND instruction last (PLAN(EntryStateOfChlld))

od

return(EntrySequenceofChild) 
active during (non-incremental) evaluation, in other words, the first state of the entry sequence for $r$. Finding this state requires knowing the entry sequence of parent $(r)$, which in turn requires knowing the entry sequence for parent $(\operatorname{parent}(r))$, and so on, all the way to the root of the tree. Because $r$ can be nested arbitrarily deeply in the tree, computing all of these entry sequences would, in general, take more than $O(|A F F E C T E D|)$ units of time; however, by storing entry sequences in the tree, we can decide in unit-time how to reactivate $r$.

We use the notation r.EntrySequence to denote the entry sequence at node $r$, and we redefine the term prepared for propagation at $r$ to mean that $r$ and each ancestor of $r$ is labeled with its entry sequence. As was the case in Chapter 5, the condition that the tree be prepared for propagation at the position of the editing cursor is an invariant for the editor that must be reestablished after cursor motions and subtree replacements. Given that the editıng cursor is positioned at node $r$, and that the tree is prepared for propagation at $r$, the cursor movement AscendToParent has the side-effect:

$$
\text { r.EntrySequence : = ne11 }
$$

The cursor movement DescendToChild( $j)$ has the side-effect:

$$
r_{j} \text { EntrySequence }:=\text { BuildEntrySequence }(r, r \text {.EntrySequence, } j \text { ) }
$$

Finally, a subtree replacement at node $r$ may change the type of production that applies at $r$, thus it is necessary to recompute the entry sequence at $\mathbf{r}$ to reestablish the invariant. 
To begin the updating process, $r$ is reactivated by initializing r.MachineState, r.PlanIndex and Reactivated as follows:

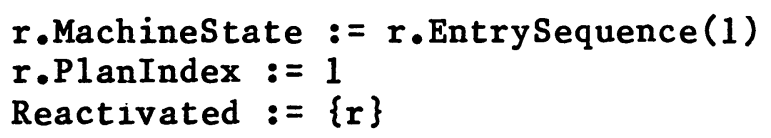

As updating progresses, VISIT and SUSPEND instructions will call for control to be transferred to neighboring machines. Whereas during nonincremental tree-walk evaluation, a $\operatorname{VISIT}(k, I)$ instruction always causes control to be transferred to the $k^{\text {th }}$ child, and a SUSPEND instruction always causes control to be transferred to the parent, during change propagation the goal is to skip over as many unnecessary VISITs and SUSPENDs as possible. In particular, transfers of control are ignored in the following two situations:

a) When the machine at node $t$ reaches a $\operatorname{VISIT}(k, I)$ instruction, if the $k^{\text {th }}$ child of $t$ is not a member of Reactivated, $t$ retains control and proceeds to the next instruction in its plan.

b) When the machine at $t$ reaches a SUSPEND instruction, if the parent of $t$ is not a member of Reactivated, $t$ retains control, finds the entry state $e$ that follows its current entry state in t.EntrySequence, and begins executing the plan for e.

When the machine that applies at node $t$ executes an EVAL instruction that changes the value of attribute $b$, all attributes that use $b$ as an argument may become inconsistent. These attributes belong to a production instance $\mathrm{p}^{\prime}$ that is one of the neighbors of the production instance at $t$. If $b$ is a synthesized attribute of $t, p^{\prime}$ is the 
production instance that derives $t$; if $b$ is an inherited attribute of a child of $t, p^{\prime}$ is the production instance derived from the child.

For inconsistent attributes in $\mathrm{p}^{\prime}$ to get reevaluated, $\mathrm{p}^{\prime}$ must be a member of Reactivated; if $\mathrm{p}^{\prime}$ is not already a member of Reactivated, it is made a member, and the correct state for the machine at $p^{\prime}$ is established by simulating all activity that has previously been suppressed at $\mathrm{p}^{\prime}$, using the procedure ReactivateNe1ghbor, given as Algorithm 9.5.

If the attribute $b$ that changed value is a synthesized attribute of $t$, the machine that applies at parent $(t)$ must be reactivated in an entry state $e$ and the PlanIndex field of parent( $t)$ must index a particular VISIT instruction in the plan for e. ReactivateNeighbor runs through all instructions of the parent machine, until it reaches the VISIT instruction that transfers control to $t$ and causes the machine at $t$ to execute the instruction $\operatorname{EVAL}(0, \mathrm{~b})$.

Alternatively, if $b$ is an inherited attribute of the $k^{\text {th }}$ child of $t$, the machine that applies at the child node must be reactivated either in the machine's initial state, if no VISITs have been skipped, or else in the quiescent state that would have been reached had all the skipped VISITs been carried out. ReactivateNeighbor runs through all instructions of the parent machine, advancing the state of the child machine on each VISIT(k, I) instruction, until the parent machine reaches the instruction $\operatorname{EVAL}(k, b)$.

In either case, the execution simulation carried out by ReactivateNeighbor is limited to the activity of a pair of machines; consequently, the time needed to reactivate a neighbor is constant for a given grammar. 


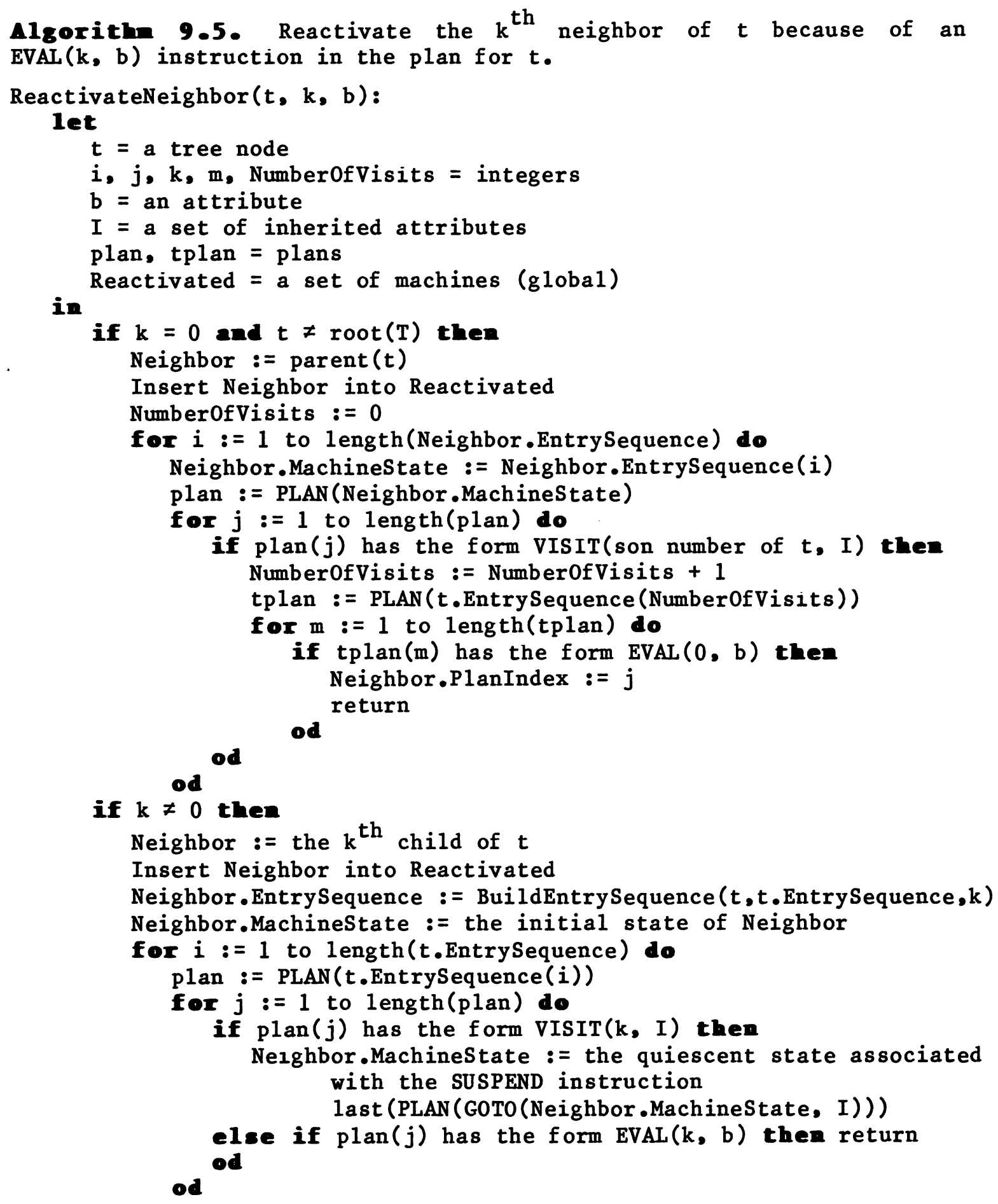


These ideas are incorporated into the change propagation algorithm given as Algorithm 9.6. This version of PROPAGATE interleaves tree-walk evaluation with calls to ReactivateNeighbor. By using ReactivateNeighbor to simulate the evaluation activity that had previously been suppressed, ReactivateNeighbor reestablishes a node's execution state so that when the node receives control it performs the rest of its instructions in the same order as it would during non-incremental evaluation. Because a machine is reactivated only when a new member of AFFECTED is identified, and because reactivating a machine by ReactivateNeighbor takes a bounded amount of work for a given grammar, the total cost of Algorithm 9.6 is $0(\mid$ AFFECTED $\mid)$.

In many respects, Algorithm 9.6 is very similar to Algorithm 5.3 , the change propagation algorithm presented in Chapter 5. Both algorithms achieve optımal behavior by ensuring that whenever an attribute is reevaluated, the value computed is the attribute's final value. Both algorithms confine their activity to the region of the tree in which attributes have changed value. Both algorithms expand this region only when they assign a new value to an attribute that is at an extremity of the region.

The chief advantage of using Algorithm 9.6 in place of Algorithm 5.3 is that storage can be used more efficiently with Algorithm 9.6 than with Algorithm 5.3. During change propagation, Algorithm 5.3 constructs a graph data-structure to represent dependencies among attributes in the portion of the tree in which attributes have changed value, and it uses a worklist to record attributes that are ready for reevaluation. In the worst case, these data structures can grow to be on the order of the 
Algorithn 9.6. Change propagation by tree-walking.

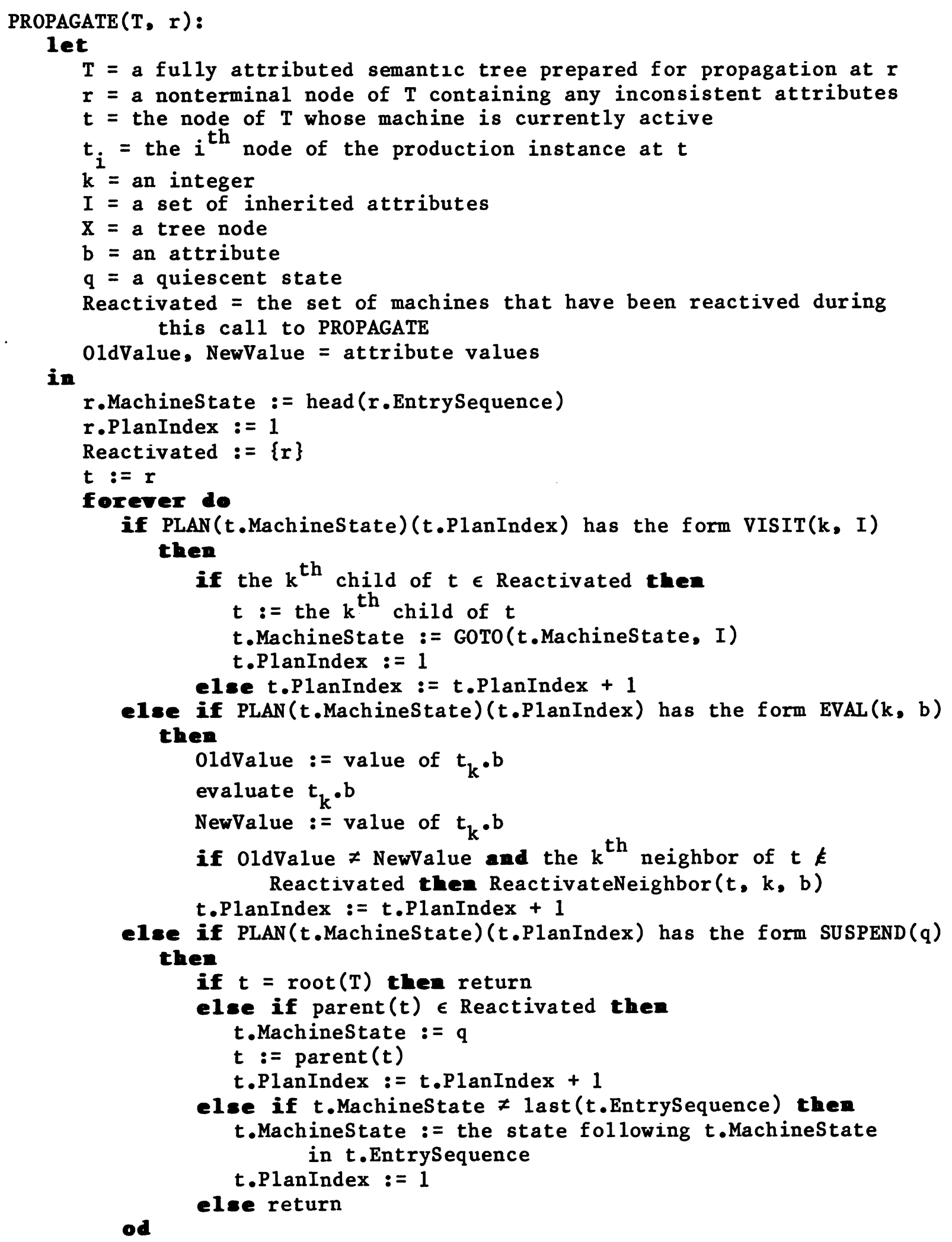


size of the semantic tree. In contrast, Algorithm 9.6 makes use of no growing data structures; in the worst-case, each tree node must be labeled with a machine state, an index into a plan, and an entry sequence.

A second advantage of using Algorithm 9.6 in place of Algorithm 5.3 is that less information needs to be stored in the tree. To apply Algorithm 5.3, each node in the tree must be labeled with one of its two characteristic graphs. To apply Algorithm 9.6, only the nodes along the path from the cursor position to the root must be labeled, not with a characteristic graph, but with an entry sequence. Because on each successive visit to a given node, at least one additional inherited attribute has been made available, and because each visit is guaranteed to make available at least one additional synthesized attribute, the size of an entry sequence for a node of type $X$ is no more than $\max (1, \min (|I(X)|,|S(X)|))$. In contrast, the characteristic graph for a node of type $X$ has at least $|I(X)|+|S(X)|$ vertices, as well as edges.

Cohen and Harry argue that in practice the number of characteristic graphs is small, and that the amount of storage required for each node can be reduced by storing the characteristic graphs in a dictionary and labeling the nodes of the tree with the key-value of the graph instead of the graph itself [Cohen \& Harry 1979]. This technique is also possible for the entry sequences used by Algorithm 9.6. 


\subsection{Incremental evaluation for ordered attribute grammars}

Under certain conditions, it is possible to store entry sequences globally instead of at nodes of the tree, and thus decrease the amount of storage used by the updating algorithm of the previous section. For the class of erdered attribute grammars, defined in [Kastens 1980], this improvement is always possible.

The key to the optimal behavior of Algorithm 9.6 is that Algorithm 9.5 reactivates a machine in unit-time. Algorithm 9.5 must search the machine's finite-state control to find the correct state in which to initialize the machine. In general, the transition diagram of a treewalk evaluation machine is a directed-acyclic graph, but because an entry sequence for the machine essentially specifies a path in the transition diagram, Algorithm 9.5 only has to consider the instructions along the path.

When the transition diagram of a machine is a simple chain of instructions with no branching, then the graph has a single path that is followed by every instance of the machine. In this case, the machine has a unique entry sequence that can be stored globally, instead of at each node where the machine applies.

For ordered attribute grammars, tree-walk evaluators can be constructed in which the machine for each production is a non-branching chain of instructions [Kastens 1980]. Consequently, we can perform optimal-time, incremental attribute evaluation for ordered attribute grammars using the algorithms of the previous section, but each node of the tree need only store a machine state and an index into a plan, and all entry sequences can be stored globally. 
A second advantage of using ordered attribute grammars instead of absolutely noncircular attribute grammars is that it is possible to test that a grammar is an ordered grammar and to construct a tree-walk evaluator for the grammar in polynomial time [Kastens 1980]; the same tasks for absolutely noncircular grammars may take exponential time, because of the need to construct the sets of characteristic graphs. 
CHAPTER 10

Some Final Remarks

Because of our previous experience developing the Cornell Program Synthesizer, we knew that the most challenging aspect of the Synthesizer Generator would be the design of the mechanism for providing contextdependent facilities. However, we were unhappy with the approach used in earlier systems for generating language-based editors, which was to build in mechanisms for a few special facilities [Hansen 1971, Yonke 1975]. The chief drawback of this approach is that new applications must inevitably run up against the limits of the system's capabilities.

On the other hand, we were also unhappy with the prospect of generalizing the method used to implement context-dependent facilities in the Synthesizer and other existing programming environments. In these systems, each editing operation that affects a node of type $\mathrm{X}$ invokes a semantic routine associated with the category $X$. A semantic routine may traverse the entire program tree, making updates to nodes of the tree as well as to global data-structures. In an editor-generating system based on this approach, an editor-designer would specify context-dependent facilities by writing a collection of semantic-action routines for updating the program tree [Medina-Mora 1982, Krafft 1981]. The chief drawback of this approach is that each action routine must explicitly specify how to make the internal program representation consistent. 
This thesis describes the use of a formal method of defining language semantics for specifying context-dependent facilities in language-based editors; it develops a framework for basing the semantic component of a language-based editor on an attribute grammar. In contrast to the methods outlined above, the declarative nature of an attribute-grammar specification frees the editor-designer from having to worry about maintaining the internal program representation in a consistent state.

Our original reason for choosing to work with attribute grammars was that they can conveniently describe the scoping rules for variable names in block-structured programming languages. Attribute grammars have proven to be a serendipitous choice because many other language relationships that can be described with attribute grammars also have useful applications in language-based programming environments. For example, [Gerhart 1975] shows how attribute grammars provide a simple way of generating verification-conditions as well as conditions needed for checking that a program transformation preserves meaning, and [Arthur \& Ramanathan 1981] shows how attribute grammars can be used to detect data-flow anomalies. We believe that this at least partly demonstrates the virtue of adopting formal semantic methods for specifying language-based editors.

\subsection{Summary of major results}

The model of editing presented in Chapter 4 provides a simple framework for discussing how attribute grammars can be used in language-based editors. In this model, programs are represented as attributed trees that are modified by subtree-replacement operations; 
incremental semantic analysis is carried out by updating attribute values in response to such restructuring operations. The updating algorithms presented in Chapter 4 provide two different ways of carrying out this process. The incremental viewpoint, and consequent concern with how to update attributes, sets this work apart from previous work on attribute grammar evaluation.

The updating algorithm developed in Chapter 5 is a considerable improvement on the algorithms of Chapter 4 because it updates a tree with as few semantic function applications as possible, within a constant factor. The number of attributes reevaluated is proportional to the number of attributes that actually need new values, so in this sense, the algorithm is asymptotically optimal in time.

The model of editing used in Chapters 4 and 5 corresponds to a derivation-tree editor in which all editing operations are simple subtree replacements. The extensions to the model of editing that are discussed in Chapter 6 allow attribute grammars to be used in other types of language-based editors. Demand attributes allow language-based editors to have facilities for supplying answers in response to a user's query. Tree operations other than subtree replacements allow at tribute grammars to be used in language-based editors that are more than just simple derivation-tree editors; for example, complex tree restructurings are needed in language-based editors that allow the user to manipulate programs as text.

The material in Chapters 4, 5, and 6 furnishes a basis for the semantic component of a language-based editor, and the methods described 
have actually been used in two prototype systems, Babel [Horton 1981b] as well as the author's Synthesizer Generator [Reps 1981].

This same material also has applications in conventional optimizing compilers for updating data-flow information after an optimization has been applied. Because optimizations rearrange or remove statements of a program, an optimization may invalidate some of the previously computed data-flow information; this information must be updated before further optimizations can be applied. However, the data-flow analys is needed for many kinds of program optimizations can be expressed with an attribute grammar. By using attributed trees as the intermediate program representation, and by expressing each optimization as a restructuring of the tree, incremental-attribute-evaluation techniques can be used to reestablish consistent global data-flow information in the program after each optimization.

The principle drawback of attribute-grammar-based systems is their rather extravagant consumption of storage. Chapters 7,8 , and 9 present some new techniques to help alleviate this problem.

Chapter 7 shows that for an attribute-grammar-based compiler, it is possible to carry out attribute evaluation while retaining only a sublinear number of attributes at any stage. Algorithm 7.4 evaluates a distinguished attribute of n-attribute tree in a linear number of steps, yet it retains only $O(\sqrt{n})$ attribute values at any stage of evaluation. Potentially, this algorithm can be employed in an attribute-grammarbased compiler to make efficient use of primary and secondary storage. Chapter 7 also gives an algorithm that evaluates a distinguished attribute of a derivation tree using no more than $O(\log n)$ values at any 
stage. This algorithm is less likely to be of practical importance because of its quadratic running time.

Chapter 8 discusses methods for sharing storage for attributes whose values are complex data structures. The use of sharable 2-3 trees for representing symbol tables allows considerable sharing of storage, and also allows lookup operations to be carried out efficiently. The method presented for implementing arrays with sharable 2-3 trees can be used to design sharable representations for a large class of data-types.

To be able to apply the optimal-time updating algorithm of Chapter 5, it is necessary to associate a characteristic graph with each node in the tree. In practice, the amount of space needed for storing these graphs is likely to be comparable to the amount of space needed for storing attribute values. Chapter 9 discusses how to reduce the storage penalty associated with being able to update the tree optimally, and develops algorithms for updating trees of four restricted classes of attribute grammars.

\subsection{Euture work}

Several of the ideas presented in this dissertation, including Algorithm 5.5, the optimal-time algorithm for updating a semantic tree, have been implemented in the Synthesizer Generator, a prototype system for generating language-based editors from an attribute-grammar description. The experimental editors that have been generated using this system demonstrate the potential of the approach for building interactive program editors, program verifiers, and program analysis tools. However, the present system is only a prototype suitable for such 
experimental studies and is far from being a finished product. Continuing development of the Generator is needed to increase its power and flexibility. The ultimate test, of course, is to make use of the system to construct a production editor for a full programming language.

In a larger context, our research is directed towards being able to generate complete programming environments for different languages, not just program editors. The methods for incremental attribute evaluation provide a suitable basis for incremental translation; however, without additional support, each environment-designer would be faced with the prospect of having to build the entire execution environment on his own. Thus, one topic for further research is to $f$ ind ways of automating the construction of facilities for execution and debugging.

We believe that the direction that should be taken by future research on program-development environments is toward systems that support particular programming methodologies; for example, one can imagine a system that supports formal reasoning about predicate transformers [Dijkstra 1976], or alternatively, a system that supports program development by the successive application of correctness-preserving transformations [Balzer et al. 1975, Gerhart 1975]. The methods developed in this dissertation make a contribution to this line of research by providing a general mechanism for maintaining the internal representation of the program in a consistent state. 


\section{APPENDIX}

The Synthesizer Generator Reference Manual

[Interim version]

\section{Introduction}

The Synthesizer Generator is a system for generating syntaxdirected editors from a language specification. Editor specifications are written in the Synthesizer Specification Language (SSL), which is basically an attribute grammar [Knuth 1968b] with certain features tailored to the use of attributed trees in syntax-directed editors. The Synthesizer Generator has two components:

a) an editor kernel that consists of an attributed-tree data-type [Demers et al. 1981, Reps 1982] and a driver for interactively manipulating attributed trees; the kernel takes input from the keyboard and executes appropriate operations on the current tree.

b) a translator, named ssl, that takes as input an SSL specification, and produces as output several files containing code and initialized tables written in C [Kernighan \& Ritchie 1978].

Section 2 gives a brief introduction to how to use an editor generated with the Synthesizer Generator. Section 3 describes how editors are specified in SSL. Section 4 describes how to invoke the Synthesizer Generator to build an editor. Section 5 gives an example of an SSL 
specification for a simple editor that enforces a few context-dependent constraints.

The Synthesizer Generator is written in $C$ and runs under Berkeley UNIX on a VAX 11/780; this manual describes the system as of August 1982. The present system is a prototype suitable for experimental studies of various applications, but it is far from being a finished product; the system needs certain extended capabilities, such as the notions of iterated nonterminals and optional nonterminals, as well as certain more basic capabilities, such as the ability to save files between editing sessions.

\section{Editing}

Each editor produced with the Synthesizer Generator enforces the syntax and static semantics of a particular language. A file is an object with hierarchical structure, not just a sequence of characters and lines. A file is displayed as a sentential form of an underlying context-free grammar, and all operations on the file are defined in terms of the file's partial derivation tree and the position of an editing cursor. The cursor is always positioned at one of the nodes of the tree, and its position is indicated on the display by highlighting the structure it dominates.

Files are edited by inserting and deleting at the position of the cursor in a screen of text. The body of text expands either horizontally or vertically, as necessary, to accommodate insertions; it contracts when items are deleted. The screen serves as a window into a file; whenever the cursor moves to a location in the file not contained 
on the screen, the file shifts automatically to include the new cursor position within the window.

Files are created top-down by deriving a sentence of the underlying context-free grammar. An empty file consists of a single node labeled with the root symbol of the grammar. Files are created by grafting new components onto the previously constructed structure. Each production in the grammar has a name that is used as a command for making a derivation according to that production. An insertion is made by typing the name of a production followed by one of the special-function keys; if the name matches the name of a production whose left-side matches the nonterminal at which the cursor is positioned, a derivation is made according to that rule.

Commands are echoed in the top line of the screen as they are typed in. The bottom four lines are reserved for help information, which consists of the name of the nonterminal at which the cursor is positioned and the names of the productions that can be applied at that position. The file is displayed in the remainder of the screen.

Using the special-function keys of the terminal, it is possible to change the position of the cursor; each cursor-control key moves the cursor according to a predefined traversal of the file's derivation tree. For want of better names, we refer to these movements as Left, 1 Right, Up, and Dorn. Despite this nomenclature, the effect of the

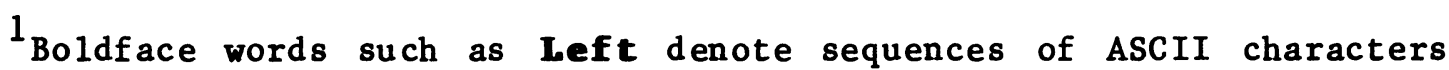
such as those transmitted by the special-function keys of a terminal. A terminal-configuration file in the user's directory defines which character sequences are interpreted as cursor-control and editing commands; a program is available to interactively define the configuration of a terminal's keyboard. 
cursor-control keys is defined with respect to the internal treestructure of a file, not the two-dimensional coordinate system of its display.

Both Right and Down move the cursor forward in the tree in left-to-right preorder, although they move the cursor in different increments; Left and $\boldsymbol{U}_{\mathbf{P}}$ are their respective inverses, and move the cursor backwards in the tree in right-to-left postorder. Up and Down both move the cursor one node at a time to the next node in the tree. Left and Right differ from $U_{p}$ and Down in that they always position the cursor at the next leaf. In each case, when the last node in the tree is reached, the traversal starts over from the beginning of the file.

Other keys move the cursor according to the nesting structure of the components of the tree. ToParent moves the cursor from a node to its parent; ToRoot moves the cursor to the root of the file tree.

Modifications to the program are accomplished by removing and replacing whole substructures of the file using the clip and Insert keys. Clip removes and saves the structure rooted at the cursor. Currently, only one clipped structure can be saved at any given time. Insert swaps this saved structure with the structure rooted at the cursor, as long as the syntactic types of their roots match. The keys Copy and InsertCopy are also used for restructuring files, by manipulating duplicate copies of structures. CopJ duplicates the structure rooted at the cursor, but does not remove the original structure from 
the tree. InsertCopy inserts a copy of the saved object at the cursor position.

An editing session is terminated by Quit.

\section{Specifying an editor}

The editor designer prepares an attribute-grammar specification that includes type definitions, declarations of nonterminals and attributes, commands and rules for making derivations, and semantic functions for evaluating attributes. The specification language SSL is syntactically very similar to YACC [Johnson 1978]. A full specification file has the form:

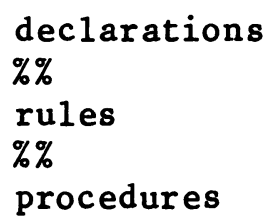

The declarations section and the procedures section may both be empty, in which case a specification is allowed to have the form:

$\% \%$

rules

Blanks, tabs, and newlines in the specification file are ignored except that they serve to delimit tokens. Comments are delimited by $n / * n$ and $n * / n$, and may appear in the file before or after any token. 


\subsection{Rules}

The rules section of a specification contains a list of grammar rules of the form:

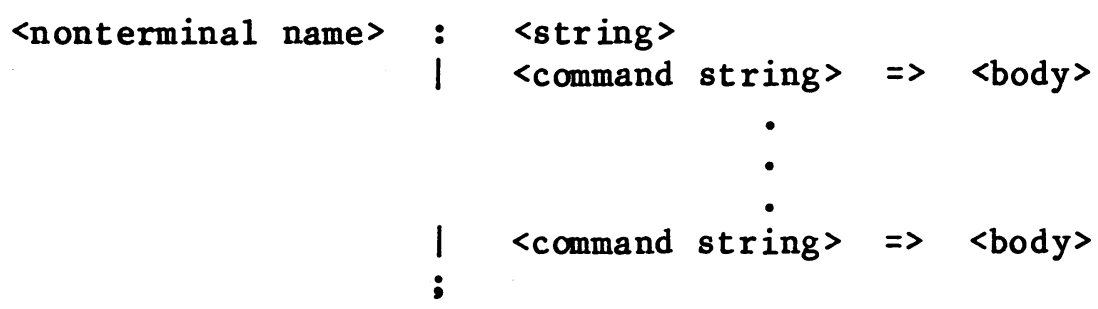

Each of the right-sides of a rule is called a template. The first template of a rule is termed the completing template, and it is the template used by default at an unexpanded nonterminal. The rest of the templates are of the form:

$$
\langle\text { command string }>\Rightarrow \text { body }>
$$

where <command string> serves to define a name for the template. The templates define the underlying context-free grammar on which editing operations are based. For example, if the cursor is positioned at the nonterminal on the left-side of the rule, a command matching one of the command strings on the right-side of the rule causes the corresponding body to be inserted into the tree. As in YACC, the left-side nonterminal of the first rule is taken to be the root symbol of the grammar. Unlike YACC, templates with the same left-side nonterminal must be grouped together.

The body of a template is a sequence of terminal strings and nonterminal names. Strings are as in $C$, enclosed in double quotes, e.g. "xxx", and C escapes are recognized. Names can be of arbitrary length and can be made up of letters, underscore, and non-initial digits. 
The strings in a template body specify the display format of the template. The display of a file is generated by a left-to-right traversal of the tree that interprets each terminal-string character. Pretty-printing is controlled by formatting-control characters that can be included in these strings. In particular, the characters $\backslash t, \backslash n, \backslash b$, and Ir have the following special meanings:

$$
\begin{aligned}
& \text { It move the left-margin one indentation unit to the right } \\
& \text { In line-feed, carriage-return to the current left-margin } \\
& \text { Ib move the left-margin one indentation unit to the left } \\
& \text { Ir carriage-return to the current left-margin }
\end{aligned}
$$

To illustrate this, consider the following fragment of a specification for a programming-language editor:

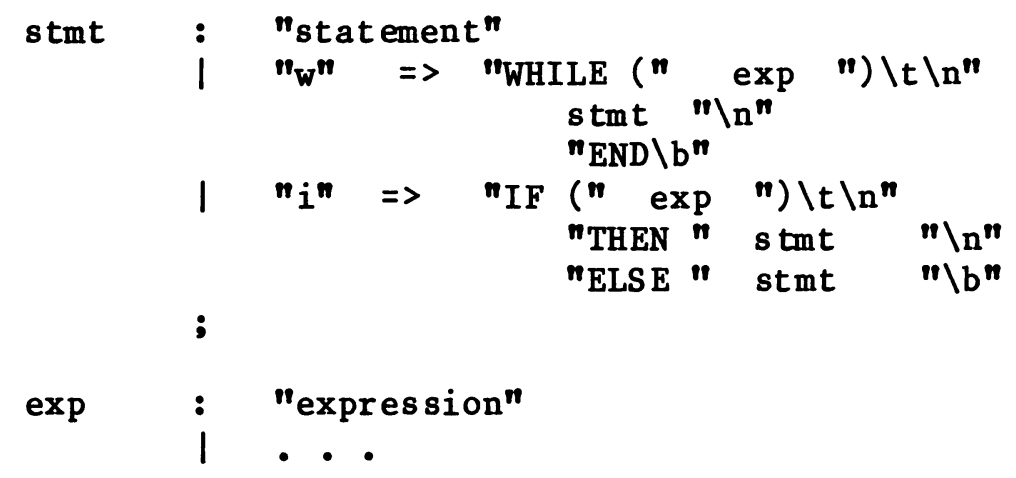

There are two points to notice about the way the formatting-control characters are used in this example. ${ }^{2}$ First, because the number of $\backslash b^{\prime} s$ in each template balances the number of $\backslash t$ 's, when a template finishes printing it has restored the indentation level back to the depth it was at when the template started printing. Second, because a $\ln$ is the initial character in the terminal string following each occurance of the

${ }^{2}$ Although in this fragment the body of each template is arranged to suggest the way in which it will be displayed, this has no affect on the actual display format. 
nonterminal stmt, a line throw is not performed at the end of each statement-template.

In the editor generated from this specification, the two statement-templates are displayed as:

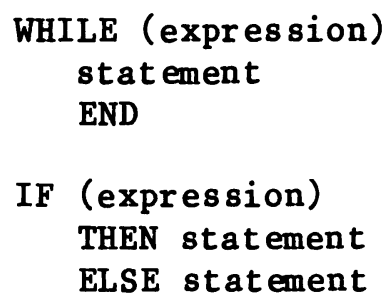

When an IF template is inserted into a WHILE template, the combined structure is displayed as:

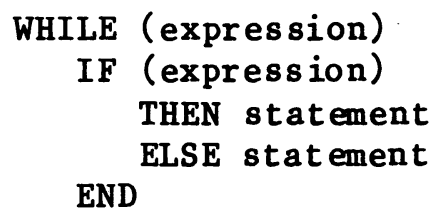

\subsection{Attributes and semantic functions}

Context-dependent features of a language can be conveniently described in an editor-specification using the semantic rules of an attribute grammar. An attribute grammar extends a context-free grammar by attaching attributes to the symbols of the grammar. Associated with each production of the grammar is a set of semantic equations; each equation defines one attribute as the value of a semantic function applied to other attributes in the production. Attributes are divided into two disjoint classes: synthesized attributes and inherited attributes; each semantic equation defines a value for a synthesized attribute of the left-side nonterminal or an inherited attribute of a rightside symbol. 
In SSL, attributes can only be attached to nonterminals, and they must be specified by a declaration statement in the initial section of the specification file. A declaration specifies the name of a nonterminal, the type of each of its attribute, and whether each attribute is synthesized or inherited. An attribute's type can either be one of the built-in types (int, char, float, double, or BOOLEAN), or a user-defined type (see below). For example, the declaration:

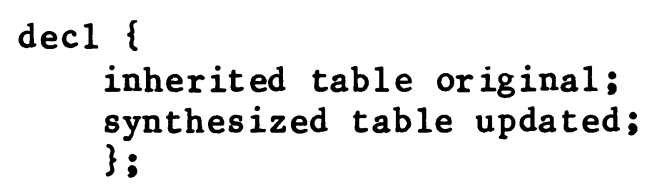

associates two attributes of the user-defined type "tablen, named original and updated, with the nonterminal decl. In such a declaration, the bracket pair defines a block; the attribute names of a nonterminal are in a separate name space from both the nonterminal names and the attribute names of the other nonterminals.

With each template or completing production, the editor designer specifies semantic equations for attributes of the nonterminals of the production. The synthesized attributes of the left-side nonterminal and the inherited attributes of the right-side nonterminals are termed the output attributes of the production; each semantic equation defines a value for a single output attribute.

A semantic equation is basically a C assignment statement except that an attribute is referred to with the selection operator ".", the syntax being:

<nonterminal name>. <attribute name>

When a production contains multiple instances of a nonterminal $X$, 
references to $X$ are disambiguated by attaching "\$n" and a number to the nonterminal name, where numbers are assigned to the instances of $X$ from left to right starting with 1 . For example, with the attributes of decl as declared above, declaration rules might be specified by:

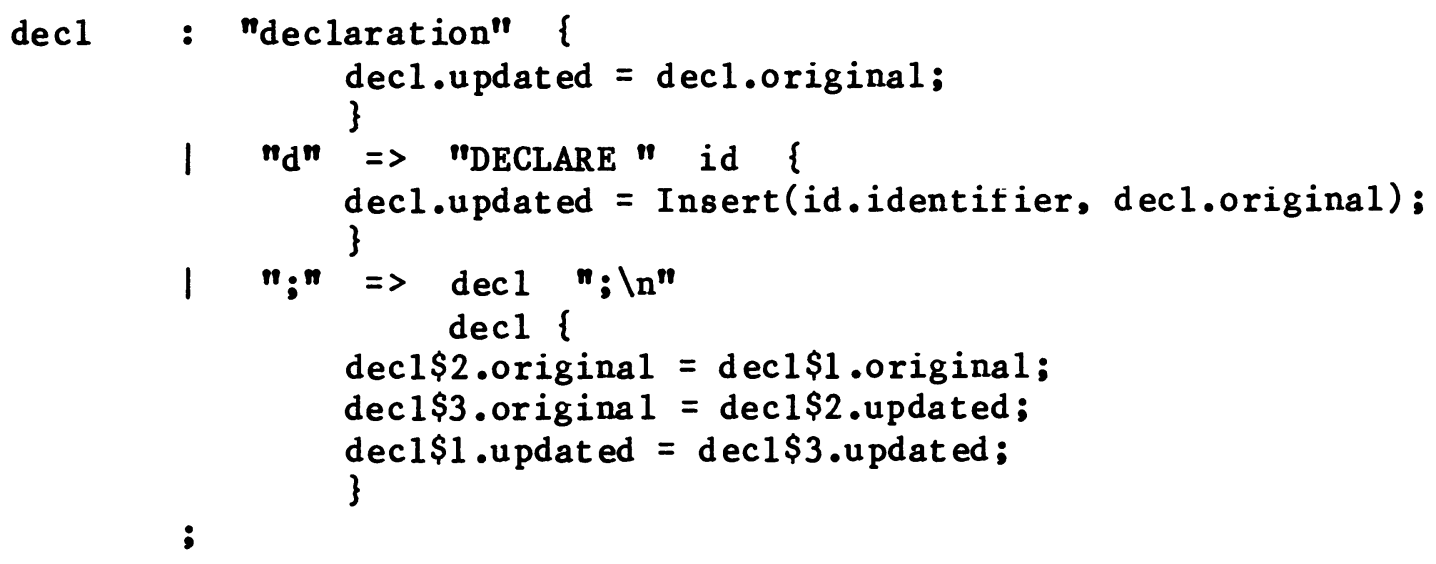

In the third template, "decl\$1" refers to the left-side nonterminal, "dec1\$2" refers to the first decl nonterminal of the right-side, and "decl\$3" refers to the second decl nonterminal of the right-side. Semantic functions other than simple expressions, such as Insert in the example above, are written in $C$ and placed in the procedures section at the end of the specification file.

The semantic equations of a specification must obey three constraints :

a) (Well-formedness) There must be equations for all output attributes of each production.

b) (Normal form) The inherited attributes of the left-side nonterminal and the synthesized attributes of the right-side nonterminals are termed the input attributes of the production. Only input attributes are allowed as arguments of a semantic function. 
c) (Noncircularity) It must not be possible to build a derivation tree in which at tributes are defined circularly.

Currently the ssl translator guards against violations of the first two constraints but not against violations of the third constraint; however, if a specification is circular, the generated editor may loop endlessly as it tries to update attribute values in the tree.

\subsection{User-defined types}

SSL has a mechanism for the editor-designer to define new types. The syntax is basically the same as the syntax of the type definition facility of C; however, with each SSL type definition, it is also necessary to provide five operations on the type. These operations are written in $C$ and they may be placed in the procedures section of the specification file. The names of these procedures are also included as part of the type definition, the syntax being:

$$
\langle\text { C-typedef }\rangle \text { with ( }\langle\text { name }\rangle,\langle\text { name }\rangle,\langle\text { name }\rangle,\langle\text { name }\rangle,\langle\text { name }\rangle) \text {; }
$$

For instance, the type "table" that occurs in several of the examples given above can be defined as a linear linked list by the type definition:

typedef struct node \{ char item; struct node $\star$ next; \} *table with (EqTable, nulle, PrTable, nulle, NilTable);

Currently, the editor kernel makes use of only the first, third, and fifth operations, so the second and fourth operations should be named "nulle". The first operation is an equality predicate for the type; it is a function of type BOOLEAN. The third operation is a 
procedure for printing the value of an attribute according the conventions of the Curses windowing package [Arnold 1981]; when this procedure is called, the first argument is a pointer to a Curses WINDOW, and the second argument is an element of the type. The fifth operation is a function that returns a special value for the type; whenever a template insertion is made, an instance of the template is created, and the special value is given to all attributes of the type that are inherited attributes of the template's left-side nonterminal.

\subsection{Further formatting control}

SSL has two facilities that provide more powerful control over the content and format of the display. One facility allows the display to include an attribute's value; the other facility allows the display to include more than one copy of a particular subtree. As a temporary measure, these facilities are provided by giving special meanings to two additional terminal-string characters.

The sequence of characters " $\backslash 001 \backslash x x x \backslash y y y "$ in a terminal string, where the $x^{\prime} s$ and $y^{\prime} s$ are digits, causes the value of attribute yyy of nonterminal $x x x$ to be generated in the display. Attributes of a nonterminal are numbered in the order of their declaration starting with 1. Nonterminals are numbered from left to right (including the left-side nonterminal) starting with 1 .

To demonstrate how this is used, consider building a programminglanguage editor that flags all declarations of variables that are also declared at an earlier point in the program. In addition to the symbol-table attributes original and updated defined previously for the 
nonterminal decl, we would give decl a synthesized string-valued attribute, named error. One of the templates on the right-side of the rule for decl would be:

\section{"DECLARE " id "\001\001 $1003 "$}

The semantic function assigning to decleerror would be:

$$
\begin{aligned}
\text { decl.error }= & \text { Member(id.identifier, decl.original) } \\
& ? \|<-- \text { multiply declared variablen } \\
& : n n ;
\end{aligned}
$$

For example, if the variable $b$ has been declared earlier, the function Member will return true, decleerror will be assigned the string n <-- multiply declared variablen, and the declaration for $b$ will be displayed as:

$$
\text { DECLARE } b<-- \text { multiply declared variable }
$$

If $b$ has not been declared earlier, decl.error will be assigned the null string, and the declaration will be displayed as:

\section{DECLARE $b$}

The sequence " $\backslash 002 \backslash x_{x x}$ causes the subtree at nonterminal $x x x$ of the production to be generated as part of the display. In this case, xxx cannot refer to the left-side nonterminal of the production because that would cause the display routine to call itself endlessly. Consequently, nonterminals of the right-side only are numbered from left to right starting with 1 .

\section{Invoking the Synthesizer Generator}

There are three steps involved in generating an editor with the Synthesizer Generator. First, the specification file is run through the 
translator ssl, which produces seven output files, named eval.cases.h, decls.h, gram.tab.h, eval.default.h, procs.h, print.h, and compare.h. Second, the files eval.c and grammar.c are compiled using the $C$ compiler. Finally, the object files grammar.o and eval.o are loaded with the object files of the editor kernel.

\section{An example}

This section gives a complete SSL specification for a programminglanguage editor with block-structured scoping of variable names. This editor detects violations of two context-dependent constraints: it flags declarations of variables that are also declared earlier in a scope, and it flags uses of undeclared variables. This example demonstrates userdefined types, control of indentation in the file display, and displaying violations of context-dependent language constraints with at tribute values.

typedef struct node \{ char item; struct node $\star$ next; \} *table with (EqTable, nulle, PrTable, nulle, NilTable);

typedef char *string with (EqString, nulle, PrString, nulle, Nilstring); $\operatorname{dec} 1\{$

inherited table original; synthesized table updated; synthesized string error; \};

id \{ synthesized char identitier; \};

stmt \{ inherited table used; synthesized string error; \}; 


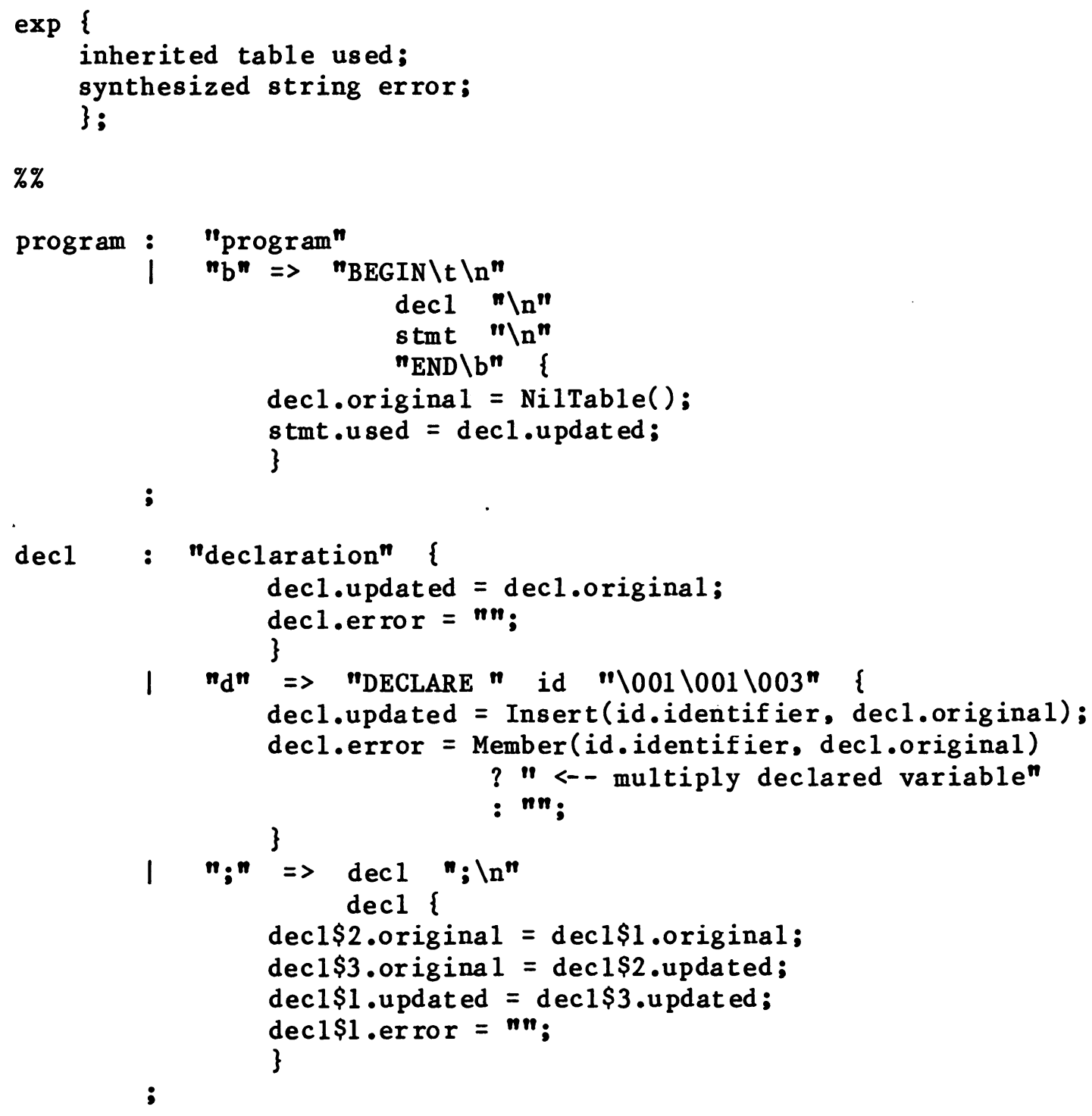




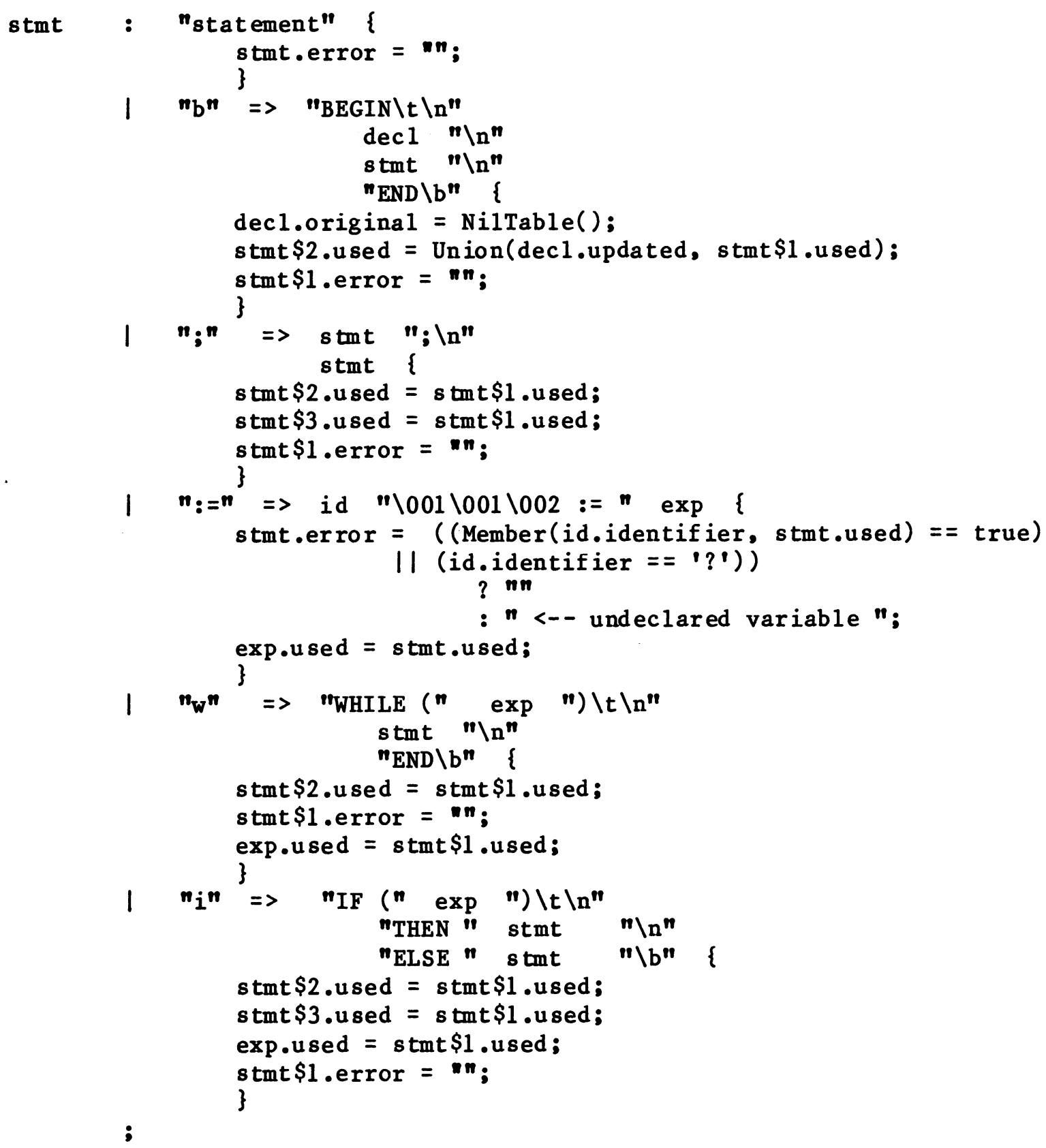


id

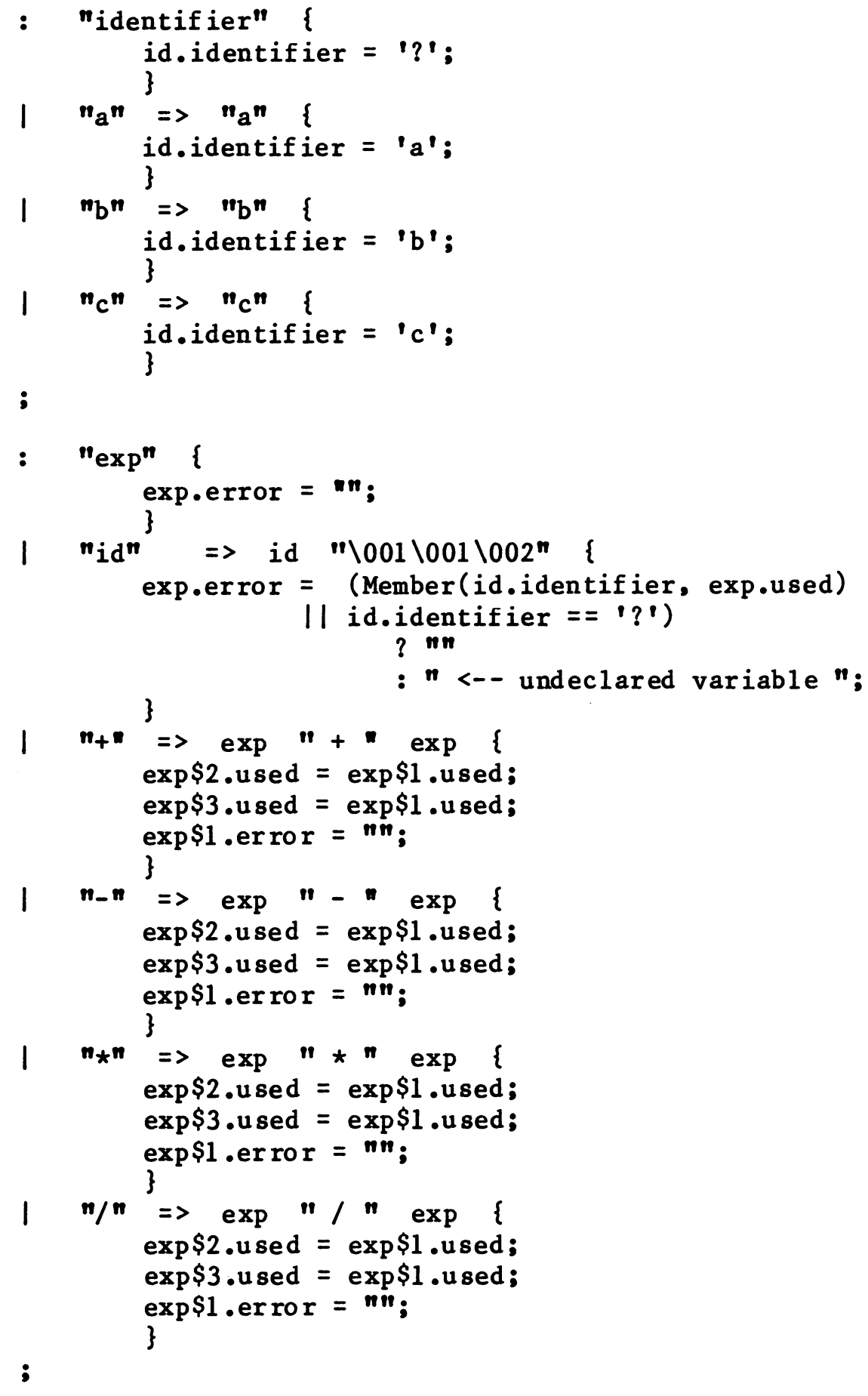




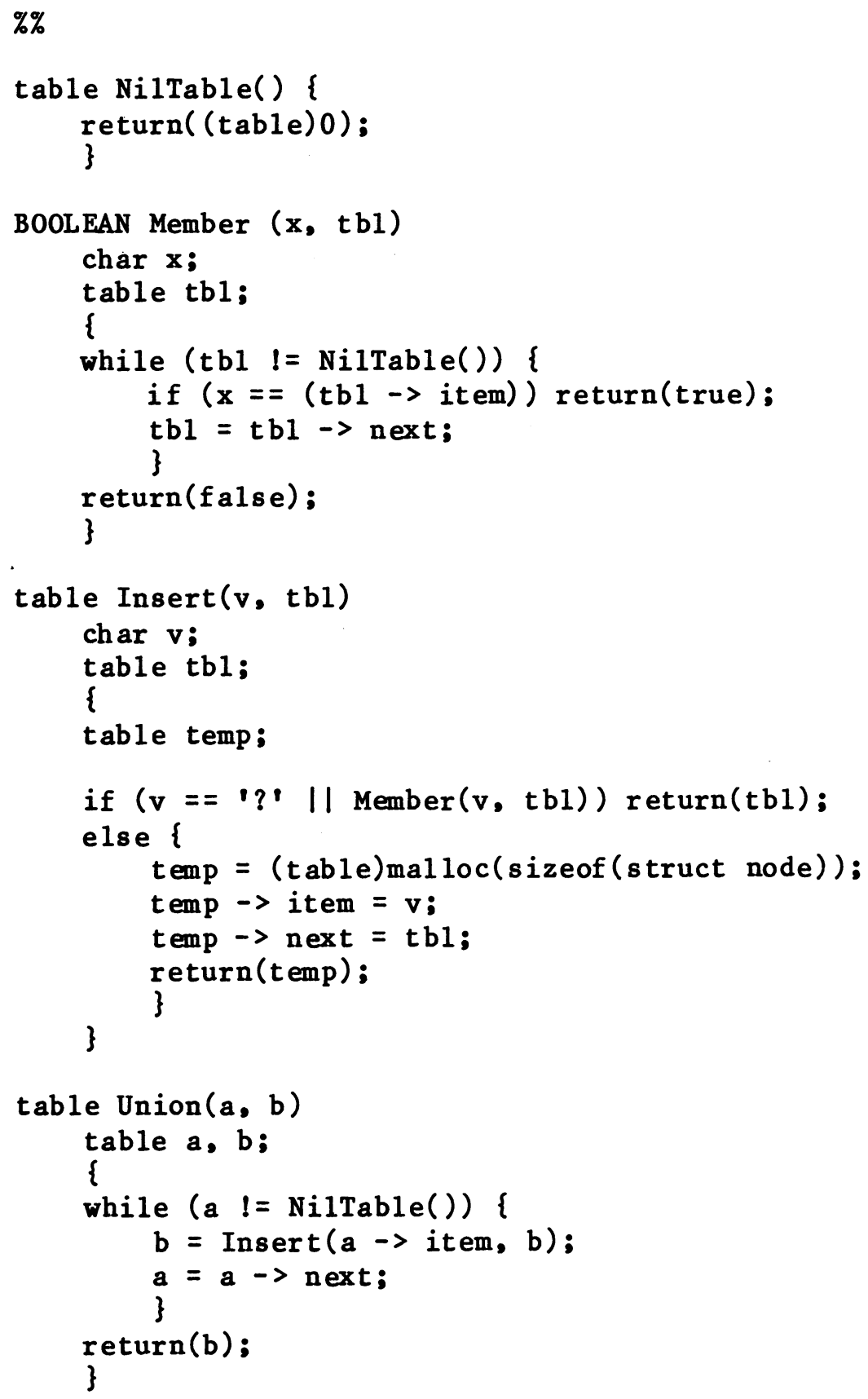




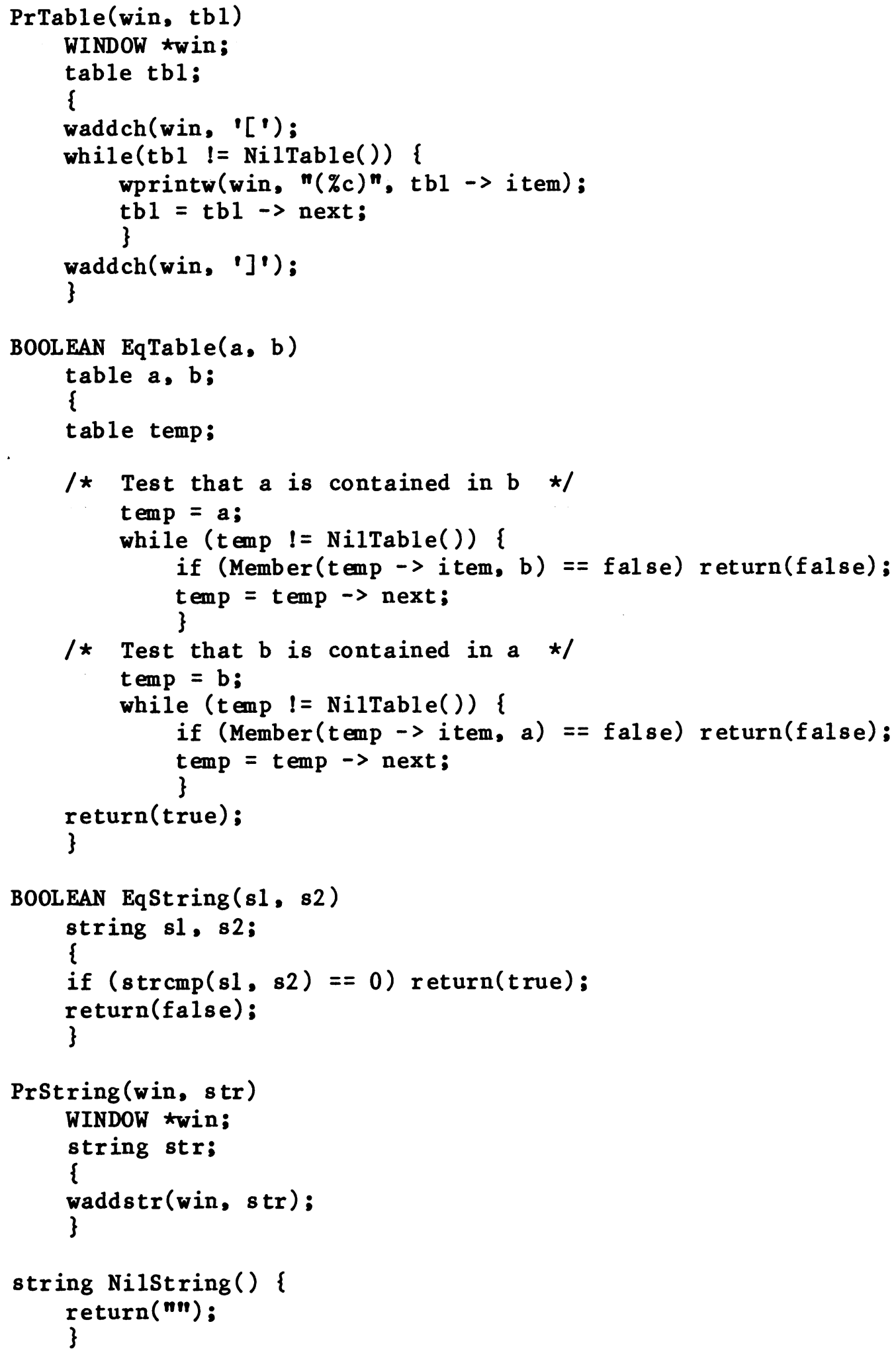




\section{BIBLIOGRAPHY}

[Aho et al. 1974]

Aho, A.V., Hopcroft, J.E., and Ullman, J.D. The Design and Analysis of Computer Algorithms. Addison-Wesley, Reading, Massachusetts, 1974 .

[Alberga et a1. 1981]

Alberga, C.N., Brown, A.L., Leeman, G.B., Mikelsons, M., and Wegman, M.N. A Program Development Tool. Conference Record of the Eighth Symposium on Principles of Programming Languages, January $1981,92-104$.

[Arnold 1981]

Arnold, K. Screen updating and cursor movement optimization: a library package. Department of Electrical Engineering and Computer Science, University of California, Berkeley, 1981.

[Arthur \& Ramanathan 1981]

Arthur, J. and Ramanathan, J. Design of analyzers for selective program analysis. IEEE Transactions on Software Engineering, SE-7, 1 (January 1981), 39-51.

[Babich \& Jazayeri 1978]

Babich, W.A. and Jazayeri, M. The method of attributes for data flow analysis. part I: exhaustive analysis, part II: demand analysis. Acta Informatica 10,3 (October 1978), 245-272.

[Balzer et al. 1975]

Balzer, R., Goldman, N., and Wile, D. On the transformational implementation approach to programming. Information Sciences Institute, University of Southern California, April 1975.

[Bochmann 1976]

Bochmann, G.V. Semantic evaluation from left to right. CACM 19, 2 (February 1976), 55-62.

[Cohen \& Harry 1979]

Cohen, R. and Harry, E. Automatic generation of near-optimal linear-time translators for non-circular attribute gramars. Conference Record of the Sixth ACM Symposium on Principles of Programming Languages, January 1979, 121-134.

[Cook 1974]

Cook, S.A. An observation on time-storage trade off. Journal of Computer and System Sciences 9, 3 (December 1974), 308-316.

[Demers et a1. 1981]

Demers, A., Reps, T., and Teitelbaum, T. Incremental evaluation for attribute grammars with application to syntax-directed editors. Conference Record of the Eighth ACM Symposium on Principles of Programming Languages, January 1981, 105-116. 
[Dijkstra 1976]

Dijkstra, E.W. A Discipline of Programming. Prentice-Hall, Englewood Cliffs, New Jersey, 1976.

[Donzeau-Gouge et al. 1975]

Donzeau-Gouge, V., Huet, G., Kahn, G., Lang B., and Levy, J.J. A structure-oriented program editor. Technical Report, INRIA, Rocquencourt, France, 1975.

[Donzeau-Gouge et al. 1980]

Donzeau-Gouge, V., Huet, G., Kahn, G., and Lang B. Programming environments based on structured editors: the MENTOR experience. Technical Report, INRIA, Rocquencourt, France, May 1980.

[Dreisbach 1972]

Dreisbach, T.A. A declarative semantic definition of PL360. Technical Report UCLA-ENG-7289, Computer Science Department, University of California, October 1972 .

[Fang 1972]

Fang, I. FOLDS, a declarative formal language definition system. Tech. Report No. STAN-CS-72-329, Computer Science Dept., Stanford University, December 1972.

[Farrow 1977]

Farrow, R.W. Attributed grammar models for data flow analysis. Ph.D. Thesis, Department of Mathematical Sciences, Rice University, May 1977.

[Farrow 1982a]

Farrow, R.W. Experience with an attribute grammar-based compiler. Conference Record of the Ninth ACM Symposium on Principles of Programming Languages, January 1982, 95-107.

[Farrow 1982b]

Farrow, R.W. LINGUIST-86: Yet another translator writing system based on attribute grammars. Proceedings of the SIGPLAN 82 Symposium on Compiler Construction, SIGPLAN Notices 17, 6 (June 1982), 160-171.

[Fosdick \& Osterweil 1976]

Fosdick, L. and 0sterweil, L. Data flow analysis in software reliability. Computing Surveys 8, 3 (September 1976), 305-330.

[Ganapathi \& Fischer 1982]

Ganapathi, M. and Fischer, C.N. Retargetable code generation and optimization using attribute grammars. Conference Record of the Ninth ACM Symposium on Principles of Programming Languages, January 1982.

[Ganzinger 1979]

Ganzinger, H. On storage optimization for automatically generated compilers. In Theoretical Computer Science, Fourth GI Conference, K. Weihrauch (ed.), Lecture Notes in Computer Science No. 67, Springer-Verlag, Berlin-Heidelberg-New York, 1979, 132-141. 
[Ganzinger et al 1977]

Ganzinger, H., Ripken, $\mathrm{K}$, and Wilhelm, R. Automatic generation of optimizing multipass compilers. Information Processing 77 , Proceedings of the IFIP Congress 77, B. Gilchrist (ed.), NorthHolland, Amsterdam-New York-Oxf ord, 1977, 535-540.

[Gerhart 1975]

Gerhart, S.L. Correctness-preserving program transformations. Conference Record of the Second ACM Symposium on Principles of Programming Languages, January $1975,54-66$.

\section{[Gillett 1977]}

Gillett, W. Iterative global flow techniques for detecting program anomalies. Ph.D Thesis, Department of Computer Science, University of Illinols at Urbana-Champaign, January 1977.

[Goto 1974]

Goto, E. Monocopy and associative algorithms in an extended LISP. Information Science Laboratory Technical Report 74-03, University of Tokyo, May 1974.

[Hansen 1971]

Hansen, W. Creation of hierarchic text with a computer display. Ph.D Thesis, Computer Science Dept., Stanford University, June 1971.

[Harary 1969]

Harary, F. Graph Theory. Addison-Wesley, Reading, Massachusetts, 1969.

[Hopcroft et al. 1977]

Hopcroft, J.E, Paul, W.J., and Valiant, L.G. On time versus space. JACM 24, 2 (April 1977), 332-337.

[Horton 1981a]

Horton, M.R. Private communication. June 1981.

[Horton 1981b]

Horton, M.R. Design of a multi-language editor with static error detection capabilities. Ph.D. Thesis, University of California, Berkeley, July 1981 .

[Jalili \& Gallier 1982]

Jalili, F. and Gallier, J. Building friendly parsers. Conference Record of the Ninth ACM Symposium on Principles of Programming Languages, January 1982, 196-206.

[Jazayeri \& Pozef sky 1977]

Jazayeri, M. and Pozefsky, D. Algorithms for efficient evaluation of multi-pass attribute grammars without a parse tree. Report TR 77-001, Department of Computer Science, University of North Carolina, February 1977 (revised May 1979).

[Jazayeri \& Pozef sky 1981]

Jazayeri, M. and Pozefsky, D. Space-efficient storage management in an attribute grammar evaluator. TOPLAS 3, 4 (October 1981), 388-404. 
[Jazayeri et a1. 1975]

Jazayeri, M., Ogden, W.F., and Rounds, W.C. The intrinsically exponential complexity of the circularity problem for attribute grammars. Communication of the ACM 18, 12 (December 1975), 697706 .

[Johnson 1978]

Johnson, S.C. Yacc: Yet another compiler-compiler. Bell Laboratories, Murray Hill, New Jersey, 1978.

[Kastens 1980]

Kastens, U. Ordered attribute grammars. Acta Informatica 13, 3 $(1980), 229-256$.

[Kastens et al.]

Kastens, U., Hutt, B., and Zimmermann, E. GAG: A practical compiler generator. Lecture Notes in Computer Science, SpringerVerlag, BerLin-Heidelberg-New York, (forthcoming).

[Kennedy \& Ramanathan 1979]

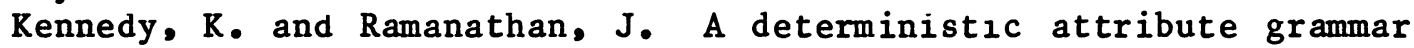
evaluator based on dynamic sequencing. ACM Transactions on Programming Languages and Systems 1, 1 (July 1979), 142-160.

[Kennedy \& Warren 1976]

Kennedy, K. and Warren, S.K. Automatic generation of efficient evaluators for attribute grammars. Conference Record of the Third ACM Symposium on Principles of Programming Languages, January 1976, 32-49.

[Kernighan \& Ritchie 1978]

Kernighan, B.W. and Ritchie, D.M. The $c$ Programing Language. Prentice-Ha11, Englewood Cliffs, New Jersey, 1978.

[Knuth 1968a]

Knuth, D.E. The Art of Computer Programming, Ve1. I: Eundamental Algorithms. Addison-Wesley, Reading, Mass., 1968, 258-268.

[Knuth 1968b]

Knuth, D.E. Semantics of context-free languages. Mathematical Systems Theory 2, 2 (June 1968), 127-145.

[Knuth 1971]

Knuth, D.E. Semantics of context-free languages: correction. Mathematical Systems Theory 5, 1 (March 1971), 95-96.

[Kratft 1981]

Krafft, D. AVID: A system for the interactive development of verifiably correct programs. Ph.D Thesis, Department of Computer Science, Cornell University, August 1981.

[Lewis et a1. 1974]

Lewis, P.M., Rosenkrantz, D.J., and Stearns, R.E. Attributed translations. Journal of Computer and Systems Sciences 9, 3 (December 1974), 279-307.

[Lindstrom 1973]

Lindstrom, G. Scanning list structures without stacks or tag bits. Information Processing Letters 2, 2 (June 1973), 47-51. 
[Lipton \& Tarjan 1977]

Lipton, R.J. and Tarjan, R.E. Applications of a planar separator theorem. Eighteenth IEEE Symposium on Foundations of Computer Science, November $1977,162-169$.

[Lorho 1977]

Lorho, B. Semantic attributes processing in the system DELTA. In Methods of Algorithmic Language Implementation, A. Ershov and C.H.A. Koster (eds.), Springer-Verlag Lecture Notes in Computer Science No. $47,21-40$.

[Masinter 1980]

Masinter, L.M. Global program analysis in an interactive environment. Xerox PARC Report SSL-80-1, January 1980.

[Medina-Mora 1982]

Medina-Mora, R. Syntax-directed editing: Towards integrated programming environments. Ph.D Thesis, Department of Computer Science, Carnegie-Mellon University, March 1982.

[Medina-Mora \& Feiler 1981]

Medina-Mora, R. and Feiler, P. An incremental programming environment. IEEE Transactions on Software Engineering SE-7, 5 (September $1981), 472-482$.

[Medina-Mora \& Notkin 1981]

Medina-Mora, R. and Notkin, D.S. ALOE users' and implementors' guide. CMU-CS-81-145, Department of Computer Science, CarnegieMellon University, November 1981.

[Mikelsons \& Wegman 1980]

Mikelsons, M. and Wegman, M.N. PDE1L: The PL1L program development environment principles of operation. Research Report RC8513, IBM Watson Research Center, Yorktown Heights, November 1980.

[Milton et al. 1979]

Milton, D.R., Kirchhoff, L.W., and Rowland, B.R. An ALL(1) compiler generator. Proceeding of the SIGPLAN Symposium on Compiler Construction, SIGPLAN Notices 14, 8 (August 1979), 152-157.

[Morris \& Schwartz 1981]

Morris, J.M. and Schwartz, M.D. The design of a language-directed editor for block-structured languages. Proceedings of the ACM SIGPLAN-SIGOA Symposium on Text Manipulation, SIGPLAN Notices 16,6 (June 1981), 28-33.

[Neel \& Amirchahy 1974]

Neel, D. and Amirchahy, M. Semantic attributes and improvement of generated code. Proceedings of the ACM 1974 Annual Conference, November 1974, 1-10.

[Paterson \& Hewitt 1970]

Paterson, M.S. and Hewitt, C.E. Comparative schematology. Record of the Project MAC Conference on Concurrent Systems and Parallel Computation, June $1970,119-127$. 
[Paul et al. 1977]

Paul, W.J., Tarjan, R.E., and Celoni, J.R. Space bounds for a game on graphs. Mathematical Systems Theory 10, 3 (1977), 239-251.

[Pippenger 1980]

Pippenger, N. Pebbling. Proceedings of the Fifth IBM Symposium on Mathematical Foundations of Computer Science, Academic and Scientific Programs, IBM Japan, May 1980.

[Räihä 1979]

Räihä, K.-J. Dynamic allocation of space for attribute instances in multi-pass evaluators of attribute grammars. Proceedings of the SIGPLAN Symposium on Compiler Construction, SIGPLAN Notices 14, 8 (August 1979), 26-38.

[Räihä 1980]

Räihä, K.-J. Bibliography on attribute grammars. SIGPLAN Notices 15, 3(March 1980), 35-44.

[Räihä et al 1978]

Räihä, K.-J., Saarinen, M•, Soisalon-Soininen, E., and Tienari, M. The compiler writing system HLP (Helsinki Language Processor). Report A-1978-2, Department of Computer Science, Unıversity of He1sinki, March 1978.

[Reps 1981]

Reps, T. The Synthesizer Editor Generator: Reference Manual. Department of Computer Science, Cornell University, September 1981.

[Reps 1982]

Reps, T. Optimal-time incremental semantic analysis for syntaxdirected editors. Conference Record of the Ninth ACM Symposium on Principles of Programming Languages, January 1982, 169-176.

[Rosen 1980]

Rosen, B.K. Monoids for rapid data flow analysis. SIAM Journal of Computing 9, 1 (February 1980), 159-196.

[Sassa and Goto 1976]

Sassa, M. and Goto, E. A hashing method for fast set operations. Information Processing Letters 5, 2 (June 1976), 31-34.

[Schulz 1976]

Schulz, W.A. Semantic analysis and target language synthesis in a translator. Ph.D. Thesis, University of Colorado, Boulder, Colorado, 1976.

[Stallman 1981]

Stallman, R.M. EMACS, the extensible, customizable selfdocumenting display editor. Proceedings of the ACM SIGPLAN-SIGOA Symposium on Text Manipulation, SIGPLAN Notices 16, 6 (June 1981), 147-156.

[Swamy \& Savage 1979]

Swamy, S. and Savage, J.E. Space-time tradeotfs for linear recursion. Sixth ACM Symposium on Principles of Programming Languages, 135-142. 
[Teitelbaum \& Reps 1981]

Teitelbaum, T. and Reps, T. The Cornell Program Synthesizer: a syntax-directed programming environment. Communications of the ACM 24, 9 (September 1981), 563-573.

[Uh1 et a1. 1982]

Uhl, J., Drossopoulou, S., Persch, G., Goos, G. Dausmann, M., Winterstein, G., and Kirchgässner, W. An attribute grammar for the semantic analysis of Ada. Lecture Notes in Computer Science No. 139, Springer-Verlag, Berlin-Heidelberg-New York, 1982.

[Warren 1975]

Warren, S.K. The efficient evaluation of attribute grammars. M.A. Thesis, Department of Mathematical Sciences, Rice University, April 1975.

[Warren 1976]

Warren, S.K. The coroutine model of attribute grammar evaluation. $\mathrm{Ph} . \mathrm{D}$. Thesis, Department of Mathematical Sciences, Rice University, April 1976.

[Watt 1979]

Watt, D.A. An extended attribute grammar for Pascal. SIGPLAN Notices 14, 2 (February 1979), 60-74.

[Wilcox et al. 1976]

Wilcox, T.R., Davis, A.M., and Tindall, M.H. The design and implementation of a table driven, interactive diagnostic programing system. Communications of the ACM 19, 11 (November 1976), 609-616.

[Wilner 1971]

Wilner, W.T. Declarative semantic definition as illustrated by a definition of Simula 67. Ph.D. Thesis, Computer Science Department, Stanford University, June 1971 .

[Winograd 1975]

Winograd, T. Breaking the complexity barrier again. Proceedings of the ACM SIGPLAN-SIGIR Interface Meeting, SIGPLAN Notices 10,1 (January 1975), 13-22.

[Wood 1981]

Wood, S.R. Z - The 95\% text editor. Proceedings of the ACM SIGPLAN-SIGOA Symposium on Text Manipulation, SIGPLAN Notices 16,6 (June 1981), 1-7.

[Yonke 1975]

Yonke, M.D. A knowledgeable, language-independent system for program construction and modification. ISI/RR-75-42, Information Sciences Institute, Unıversity of Southern California, October 1975. 
INDEX OF SYMBOLS

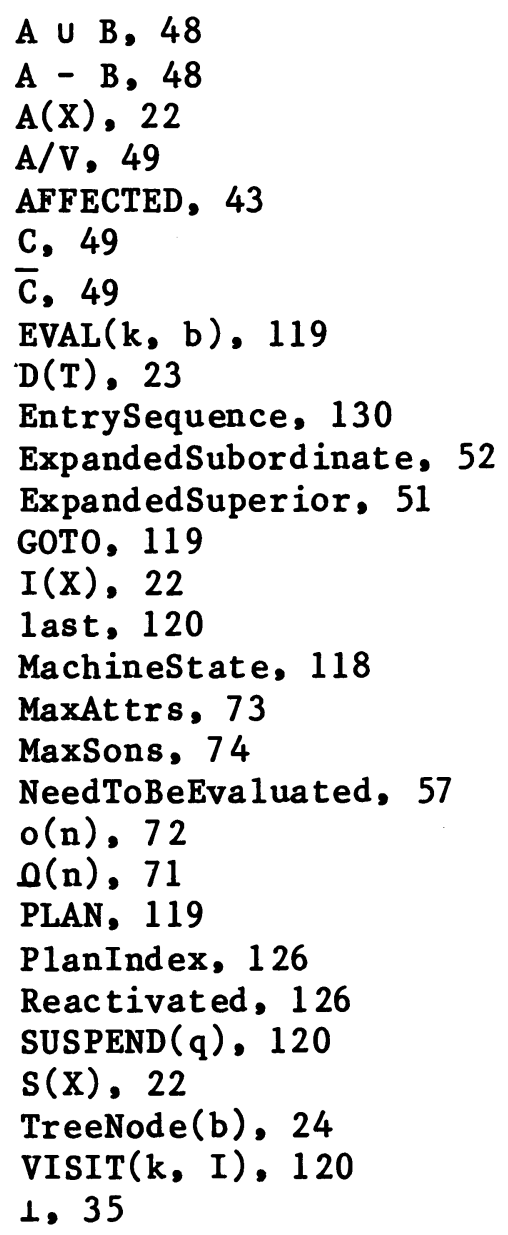


pruning operation, 35

quiescent state, 118

ready for evaluation, 32

semantic equation, 22

function, 22

tree, 24

separat or set, 74

sharable 2-3 tree, 105

split operation, 18

subordinate characteristic graph, 49

subtree replacement, 36

superior characteristic graph, 49

synthesized attribute, 22

topological evaluation, 46

tree, fully-attributed, 24

semantic, 24

sharable 2-3, 105

tree-walk evaluation, 117

order, 125

two-phase incremental

evaluation, 42

union, of two graphs, 48

update, 115

well-formed at tribute

grammar, 25 